\title{
C-H Bond Functionalization of Benzoxazoles with Chromium(0) Fischer Carbene Complexes
}

\author{
Fangdong Hu, ${ }^{\dagger}, J^{\dagger}$ Jinghui Yang, ${ }^{\dagger}$ Ying Xia,${ }^{\dagger}$ Chen Ma,${ }^{\dagger}$ Haiping Xia, ${ }^{\S}$ Yan Zhang, ${ }^{\dagger}$ Jianbo \\ Wang ${ }^{*} \dagger$ \\ ${ }^{\dagger}$ Beijing National Laboratory of Molecular Sciences (BNLMS) and Key Laboratory of \\ Bioorganic Chemistry and Molecular Engineering of Ministry of Education, College of \\ Chemistry, Peking University Beijing 100871, China \\ School of Chemistry and Chemical Engineering, Shandong University, Jinan 250100, China \\ ${ }^{\S}$ College of Chemistry and Chemical Engineering, Xiamen University, Xiamen, 361005, China \\ Email:wangjb@pku.edu.cn
}

\section{CONTENTS}

1. Spectral data of the substrates................................................... S1

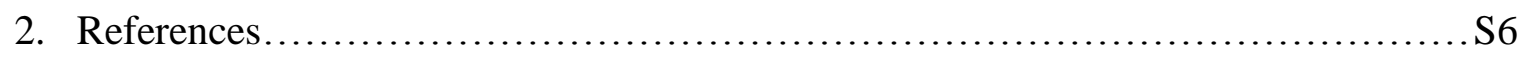

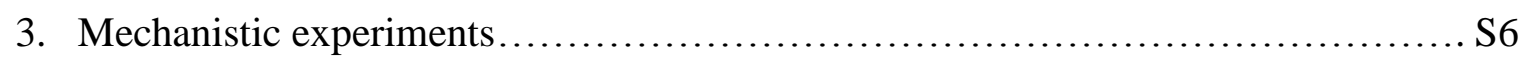

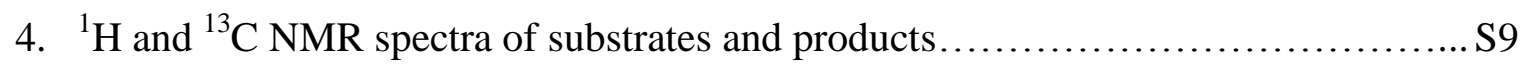




\section{Spectral data for the substrates}

\section{4-Methylbenzo[d]oxazole (1b) ${ }^{1}$}

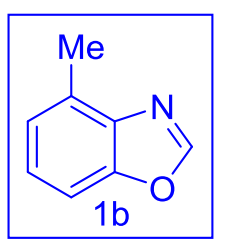

${ }^{1} \mathrm{H}$ NMR $\left(400 \mathrm{MHz}, \mathrm{CDCl}_{3}\right) \delta 8.07(\mathrm{~s}, 1 \mathrm{H}), 7.40(\mathrm{~d}, J=8.2 \mathrm{~Hz}, 1 \mathrm{H}), 7.28(\mathrm{t}, J$ $=7.6 \mathrm{~Hz}, 1 \mathrm{H}), 7.16(\mathrm{~d}, J=7.5 \mathrm{~Hz}, 1 \mathrm{H}), 2.64(\mathrm{~s}, 3 \mathrm{H}) .{ }^{13} \mathrm{C} \mathrm{NMR}(100 \mathrm{MHz}$, $\left.\mathrm{CDCl}_{3}\right) \delta 151.67,149.67,139.21,130.97,125.22,125.00,108.18,16.38$.

\section{5-tert-Butylbenzo[d]oxazole (1d) ${ }^{3}$}

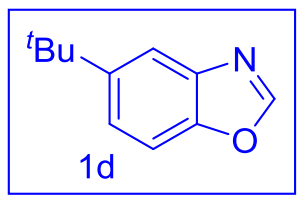

${ }^{1} \mathrm{H}$ NMR $\left(400 \mathrm{MHz}, \mathrm{CDCl}_{3}\right) \delta 8.06(\mathrm{~s}, 1 \mathrm{H}), 7.81(\mathrm{~d}, J=1.6 \mathrm{~Hz}, 1 \mathrm{H})$, 7.51-7.44 (m, 2H), $1.39(\mathrm{~s}, 9 \mathrm{H}) .{ }^{13} \mathrm{C}$ NMR $\left(100 \mathrm{MHz}, \mathrm{CDCl}_{3}\right) \delta$ 152.58, $148.10,147.93,139.92,123.34,116.94,110.02,34.88,31.72$.

\section{5-Methoxybenzo[d]oxazole (1e) ${ }^{1}$}

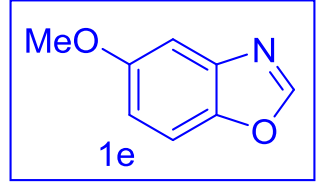

${ }^{1} \mathrm{H}$ NMR (400 MHz, $\left.\mathrm{CDCl}_{3}\right) \delta 8.06(\mathrm{~s}, 1 \mathrm{H}), 7.46(\mathrm{~d}, J=8.9 \mathrm{~Hz}, 1 \mathrm{H})$, 7.27-7.26 (m, 1H), $6.99(\mathrm{dd}, J=8.9 \mathrm{~Hz}, 2.5 \mathrm{~Hz}, 1 \mathrm{H}), 3.86(\mathrm{~s}, 3 \mathrm{H}) .{ }^{13} \mathrm{C}$ NMR $\left(100 \mathrm{MHz}, \mathrm{CDCl}_{3}\right) \delta 157.33,153.20,144.56,140.84,114.46$, $111.05,103.09,55.89$.

\section{5-Fluorobenzo[d]oxazole (1f) ${ }^{4}$}

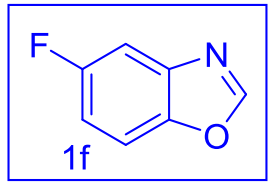

${ }^{1} \mathrm{H}$ NMR (400 MHz, $\left.\mathrm{CDCl}_{3}\right) \delta 8.13(\mathrm{~s}, 1 \mathrm{H}), 7.53(\mathrm{dd}, J=8.9 \mathrm{~Hz}, 4.2 \mathrm{~Hz}$, $1 \mathrm{H}), 7.48(\mathrm{dd}, J=8.3 \mathrm{~Hz}, 2.5 \mathrm{~Hz}, 1 \mathrm{H}), 7.14(\mathrm{dt}, J=2.5 \mathrm{~Hz}, 9.1 \mathrm{~Hz}, 1 \mathrm{H})$. ${ }^{13} \mathrm{C} \mathrm{NMR}\left(100 \mathrm{MHz}, \mathrm{CDCl}_{3}\right) \delta 160.05\left(\mathrm{~d}, J_{C F}=240.8 \mathrm{~Hz}\right), 154.10,146.28$, $140.82\left(\mathrm{~d}, J_{C F}=13.2 \mathrm{~Hz}\right), 113.50\left(\mathrm{~d}, J_{C F}=26.4 \mathrm{~Hz}\right), 111.30\left(\mathrm{~d}, J_{C F}=10.0\right.$ $\mathrm{Hz}), 106.9\left(\mathrm{~d}, J_{C F}=25.6 \mathrm{~Hz}\right)$.

\section{6-Methylbenzo[d]oxazole (1h) ${ }^{1}$}

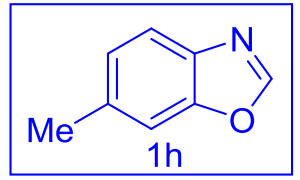

${ }^{1} \mathrm{H}$ NMR $\left(400 \mathrm{MHz}, \mathrm{CDCl}_{3}\right) \delta 8.02(\mathrm{~s}, 1 \mathrm{H}), 7.65(\mathrm{~d}, J=8.1 \mathrm{~Hz}, 1 \mathrm{H}), 7.38$ $(\mathrm{s}, 1 \mathrm{H}), 7.18(\mathrm{~d}, J=8.1 \mathrm{~Hz}, 1 \mathrm{H}), 2.50(\mathrm{~s}, 3 \mathrm{H}) .{ }^{13} \mathrm{C} \mathrm{NMR}(100 \mathrm{MHz}$, $\left.\mathrm{CDCl}_{3}\right) \delta 151.98,150.25,137.81,135.99,125.82,119.84,111.00,21.67$.

\section{6-Chlorobenzo[d] $0 x a z o l e ~(1 i)^{5}$}

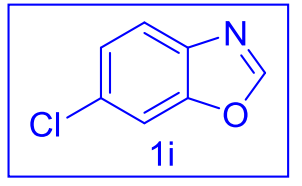

${ }^{1} \mathrm{H}$ NMR $\left(400 \mathrm{MHz}, \mathrm{CDCl}_{3}\right) \delta 8.09(\mathrm{~s}, 1 \mathrm{H}), 7.71(\mathrm{~d}, J=8.5 \mathrm{~Hz}, 1 \mathrm{H})$, 7.62-7.61 (m, 1H), $7.37(\mathrm{dd}, J=8.5 \mathrm{~Hz}, 1.8 \mathrm{~Hz}, 1 \mathrm{H}) .{ }^{13} \mathrm{C}$ NMR $(100 \mathrm{MHz}$, $\left.\mathrm{CDCl}_{3}\right) \delta 152.97,150.16,138.80,131.44,125.40,121.13,111.63$.

\section{7-Methylbenzo[d]oxazole $(\mathbf{1 j})^{6}$}

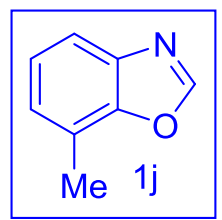

${ }^{1} \mathrm{H}$ NMR (400 MHz, $\left.\mathrm{CDCl}_{3}\right) \delta 8.09(\mathrm{~s}, 1 \mathrm{H}), 7.62(\mathrm{~d}, J=7.9 \mathrm{~Hz}, 1 \mathrm{H}), 7.27(\mathrm{t}, J$ $=7.6 \mathrm{~Hz}, 1 \mathrm{H}), 7.18(\mathrm{~d}, J=7.4 \mathrm{~Hz}, 1 \mathrm{H}), 2.55(\mathrm{~s}, 3 \mathrm{H}) .{ }^{13} \mathrm{C} \mathrm{NMR}(100 \mathrm{MHz}$, $\left.\mathrm{CDCl}_{3}\right) \delta 152.20,149.20,139.56,126.42,124.47,121.54,117.81,15.13$. 


\section{7-Bromobenzo[d]oxazole $(1 \mathrm{k})^{7}$}

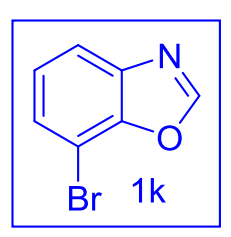

${ }^{1} \mathrm{H}$ NMR (400 MHz, $\left.\mathrm{CDCl}_{3}\right) \delta 8.15(\mathrm{~s}, 1 \mathrm{H}), 7.74(\mathrm{~d}, J=8.5 \mathrm{~Hz}, 1 \mathrm{H}), 7.55(\mathrm{~d}$, $J=7.9 \mathrm{~Hz}, 1 \mathrm{H}), 7.27(\mathrm{t}, J=8.0 \mathrm{~Hz}, 1 \mathrm{H}) .{ }^{13} \mathrm{C} \mathrm{NMR}\left(100 \mathrm{MHz}, \mathrm{CDCl}_{3}\right) \delta$ $152.42,148.26,140.73,128.81,125.83,119.71,102.97$.

Pentacarbonyl[(methoxy)(phenyl)carbene $] \operatorname{chromium}(\mathbf{0})(\mathbf{2 a})^{8}$

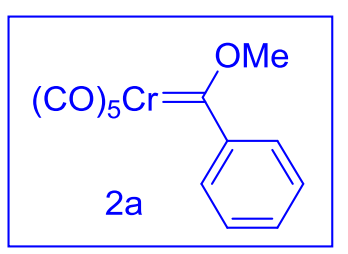

${ }^{1} \mathrm{H}$ NMR $\left(400 \mathrm{MHz}, \mathrm{CDCl}_{3}\right) \delta$ 7.41-7.29 (m, 5H), $4.70(\mathrm{~s}, 3 \mathrm{H}) .{ }^{13} \mathrm{C}$ NMR $\left(100 \mathrm{MHz}, \mathrm{CDCl}_{3}\right) \delta 351.04,224.08,216.13,153.71,130.34$, 128.17, 123.00, 67.12.

Pentacarbonyl[(2-methylphenyl)(methoxy)carbene $]$ chromium $(\mathbf{0})(\mathbf{2 b})^{8}$

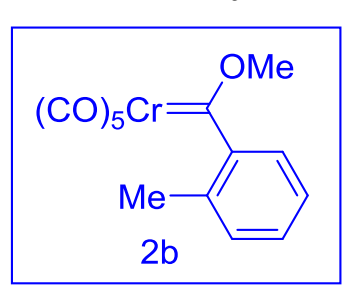

${ }^{1} \mathrm{H}$ NMR (400 MHz, $\left.\mathrm{CDCl}_{3}\right) \delta$ 7.25-7.18 (m, 3), 6.85 (s, 1H), $4.20(\mathrm{~s}$, $3 \mathrm{H}), 2.14(\mathrm{~s}, 3 \mathrm{H}) .{ }^{13} \mathrm{C}$ NMR $\left(100 \mathrm{MHz}, \mathrm{CDCl}_{3}\right) \delta 359.44,224.38$, 215.93, 130.65, 128.32, 128.18, 126.05, 125.90, 120.38, 65.55, 18.73.

Pentacarbonyl[(methoxy)(2-methoxyphenyl)carbene $]$ chromium (2c) $)^{9}$

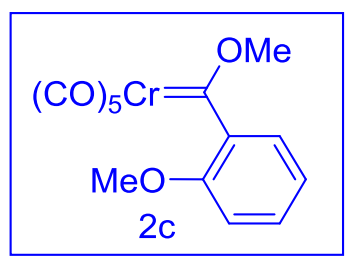

${ }^{1} \mathrm{H}$ NMR $\left(400 \mathrm{MHz}, \mathrm{CDCl}_{3}\right) \delta 7.28(\mathrm{~s}, 1 \mathrm{H}), 7.04-6.91(\mathrm{~m}, 2 \mathrm{H}), 6.79$ (s, $1 \mathrm{H}), 4.15$ (s, 3H), $3.82(\mathrm{~s}, 3 \mathrm{H}) .{ }^{13} \mathrm{C} \mathrm{NMR}\left(100 \mathrm{MHz}, \mathrm{CDCl}_{3}\right) \delta 354.67$, 225.12, 215.99, 148.57, 140.71, 129.64, 121.26, 120.72, 110.94, 65.25, 55.35 .

Pentacarbonyl[(3-chlorophenyl)(methoxy)carbene]chromium(0) (2d) ${ }^{10}$

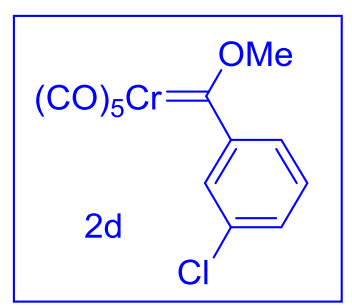

${ }^{1} \mathrm{H}$ NMR $\left(400 \mathrm{MHz}, \mathrm{CDCl}_{3}\right) \delta$ 7.36-7.16 (m, 4H), $4.74(\mathrm{~s}, 3 \mathrm{H}) .{ }^{13} \mathrm{C}$ NMR $\left(100 \mathrm{MHz}, \mathrm{CDCl}_{3}\right) \delta 348.85,223.75,215.80,154.79,134.35$, 129.91, 129.57, 122.08, 67.33.

Pentacarbonyl[(methoxy)(3-methoxyphenyl)carbene $] \operatorname{chromium}(0)(2 e)^{8}$

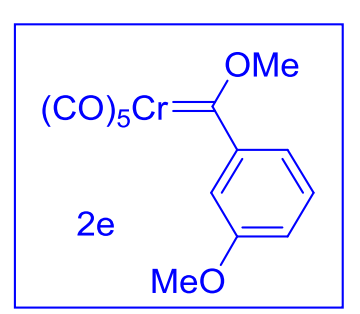

${ }^{1} \mathrm{H}$ NMR $\left(400 \mathrm{MHz}, \mathrm{CDCl}_{3}\right) \delta 7.32(\mathrm{t}, J=7.7 \mathrm{~Hz}, 1 \mathrm{H}), 6.93-6.82(\mathrm{~m}$, $2 \mathrm{H}), 6.76(\mathrm{~s}, 1 \mathrm{H}), 4.67(\mathrm{~s}, 3 \mathrm{H}), 3.84(\mathrm{~s}, 3 \mathrm{H}) .{ }^{13} \mathrm{C} \mathrm{NMR}(100 \mathrm{MHz}$, $\left.\mathrm{CDCl}_{3}\right) \delta 350.92,224.08,216.11,159.17,154.82,129.37,115.77$, 115.13, 107.99, 67.00, 55.37. 
Pentacarbonyl[(methoxy)(4-methylphenyl)carbene $]$ chromium(0) (2f) ${ }^{10}$

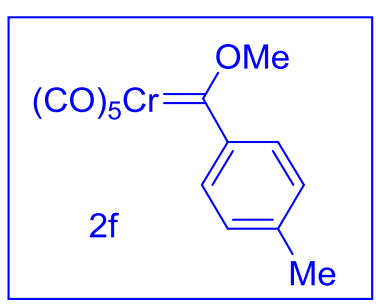

${ }^{1} \mathrm{H}$ NMR $\left(400 \mathrm{MHz}, \mathrm{CDCl}_{3}\right) \delta 7.35(\mathrm{~d}, J=6.3 \mathrm{~Hz}, 2 \mathrm{H}), 7.21(\mathrm{~d}, J=$ $6.6 \mathrm{~Hz}, 2 \mathrm{H}), 4.76$ (s, 3H), 2.39 (s, 3H). ${ }^{13} \mathrm{C} \mathrm{NMR}\left(100 \mathrm{MHz}, \mathrm{CDCl}_{3}\right)$ $\delta 348.45,224.01,216.44,151.00,141.88,128.75,124.65,67.10$, 21.41.

Pentacarbonyl[(methoxy)(4-methoxyphenyl)carbene $] \operatorname{chromium}(\mathbf{0})(\mathbf{2 g})^{10}$

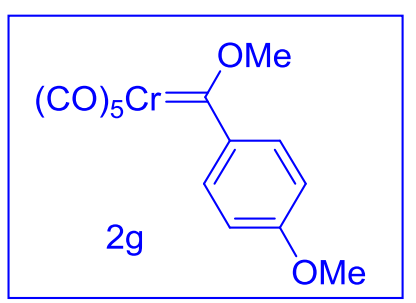

${ }^{1} \mathrm{H}$ NMR $\left(400 \mathrm{MHz}, \mathrm{CDCl}_{3}\right) \delta$ 7.75-7.74 (m, 2H), 6.92-6.91 (m, $2 \mathrm{H}), 4.86(\mathrm{~s}, 3 \mathrm{H}), 3.88(\mathrm{~s}, 3 \mathrm{H}) .{ }^{13} \mathrm{C} \mathrm{NMR}\left(100 \mathrm{MHz}, \mathrm{CDCl}_{3}\right) \delta$ 340.77, 223.72, 216.87, 163.19, 145.97, 129.58, 113.14, 67.10, 55.52 .

Pentacarbonyl[(4-biphenyl)(methoxy)carbene]chromium(0) (2h)

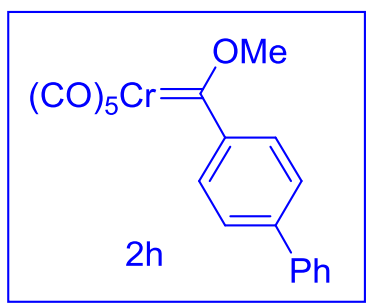

${ }^{1} \mathrm{H}$ NMR $\left(400 \mathrm{MHz}, \mathrm{CDCl}_{3}\right) \delta$ 7.64-7.40 (m, 9H), $4.79(\mathrm{~s}, 3 \mathrm{H}) .{ }^{13} \mathrm{C}$ NMR $\left(100 \mathrm{MHz}, \mathrm{CDCl}_{3}\right) \delta 348.35,224.00,216.31,152.04,143.57$, 139.64, 128.92, 128.12, 127.12, 126.67, 124.90, 67.24. IR (film): 667, $741,1218,1916,2058 \mathrm{~cm}^{-1}$. Elemental analysis calcd for $\mathrm{C}_{19} \mathrm{H}_{12} \mathrm{CrO}_{6}$ : C, 58.77; H, 3.11; found: C, 58.79; H, 3.31.

Pentacarbonyl[(4-fluorophenyl)(methoxy)carbene]chromium(0) (2i) ${ }^{10}$

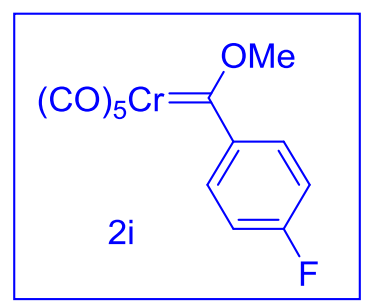

${ }^{1} \mathrm{H} \mathrm{NMR}\left(400 \mathrm{MHz}, \mathrm{CDCl}_{3}\right) \delta 7.49(\mathrm{~s}, 2 \mathrm{H}), 7.10(\mathrm{~s}, 2 \mathrm{H}), 4.82(\mathrm{~s}, 3 \mathrm{H})$. ${ }^{13} \mathrm{C} \mathrm{NMR}\left(100 \mathrm{MHz}, \mathrm{CDCl}_{3}\right) \delta 346.85,223.67,216.21,164.26\left(\mathrm{~d}, J_{C F}\right.$ $=253.8 \mathrm{~Hz}), 149.85\left(\mathrm{~d}, J_{C F}=2.0 \mathrm{~Hz}\right), 127.19\left(\mathrm{~d}, J_{C F}=8.4 \mathrm{~Hz}\right)$, $115.16\left(\mathrm{~d}, J_{C F}=21.6 \mathrm{~Hz}\right), 67.38$.

Pentacarbonyl[(4-chlorophenyl)(methoxy)carbene $]$ chromium(0) $(\mathbf{2 j})^{10}$

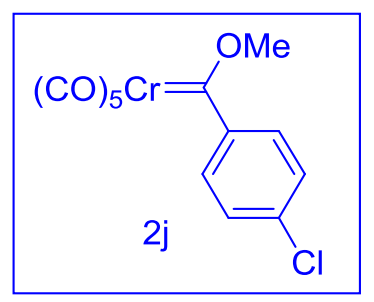

${ }^{1} \mathrm{H}$ NMR (400 MHz, $\left.\mathrm{CDCl}_{3}\right) \delta 7.39(\mathrm{~d}, J=7.7 \mathrm{~Hz}, 2 \mathrm{H}), 7.32(\mathrm{~d}, J=$ $7.3 \mathrm{~Hz}, 2 \mathrm{H}), 4.78(\mathrm{~s}, 3 \mathrm{H}) .{ }^{13} \mathrm{C} \mathrm{NMR}\left(100 \mathrm{MHz}, \mathrm{CDCl}_{3}\right) \delta 348.02$, 223.70, 216.02, 151.66, 136.99, 128.40, 125.34, 67.36.

Pentacarbonyl[(methoxy)(4-trifluoromethylphenyl)carbene $]$ chromium(0) $(2 \mathbf{k})^{10}$

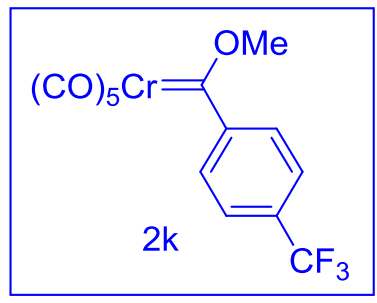

${ }^{1} \mathrm{H}$ NMR (400 MHz, $\left.\mathrm{CDCl}_{3}\right) \delta 7.68(\mathrm{~d}, J=7.4 \mathrm{~Hz}, 2 \mathrm{H}), 7.27(\mathrm{~d}, J=$ $7.9 \mathrm{~Hz}, 2 \mathrm{H}), 4.74(\mathrm{~s}, 3 \mathrm{H}) .{ }^{13} \mathrm{C} \mathrm{NMR}\left(100 \mathrm{MHz}, \mathrm{CDCl}_{3}\right) \delta 350.34$, $223.65,215.61,156.14,131.14\left(\mathrm{q}, J_{C F}=33.3 \mathrm{~Hz}\right), 125.39\left(\mathrm{q}, J_{C F}=\right.$ 
Pentacarbonyl[(3-furyl)(methoxy)carbene]chromium(0) (2l) ${ }^{11}$

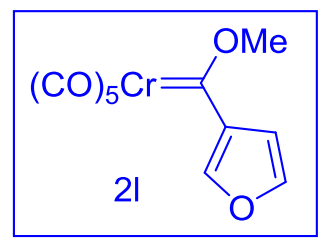

${ }^{1} \mathrm{H}$ NMR $\left(400 \mathrm{MHz}, \mathrm{CDCl}_{3}\right) \delta 8.28(\mathrm{~s}, 1 \mathrm{H}), 7.40(\mathrm{~s}, 1 \mathrm{H}), 6.70(\mathrm{~s}, 1 \mathrm{H})$, $4.84(\mathrm{~s}, 3 \mathrm{H}) .{ }^{13} \mathrm{C} \mathrm{NMR}\left(100 \mathrm{MHz}, \mathrm{CDCl}_{3}\right) \delta 327.51,222.87,216.95$, $150.71,143.57,141.89,106.95,66.40$.

Pentacarbonyl[(2-thiofuryl)(methoxy)carbene]chromium(0) (2m) ${ }^{12}$

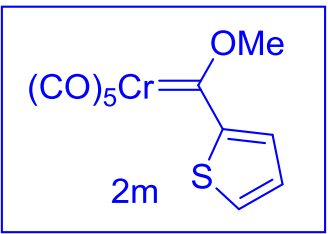

${ }^{1} \mathrm{H}$ NMR $\left(400 \mathrm{MHz}, \mathrm{CDCl}_{3}\right) \delta 8.26(\mathrm{~d}, J=2.4 \mathrm{~Hz}, 1 \mathrm{H}), 7.70(\mathrm{~d}, J=4.0$ $\mathrm{Hz}, 1 \mathrm{H}), 7.25-7.22(\mathrm{~m}, 1 \mathrm{H}), 4.84(\mathrm{~s}, 3 \mathrm{H}) .{ }^{13} \mathrm{C} \mathrm{NMR}\left(100 \mathrm{MHz}, \mathrm{CDCl}_{3}\right)$ $\delta 318.77,223.13,217.01,155.21,141.25,134.99,129.00,66.17$.

Pentacarbonyl[methoxy(2-naphthalenyl)carbene]chromium(0) (2n) ${ }^{11}$

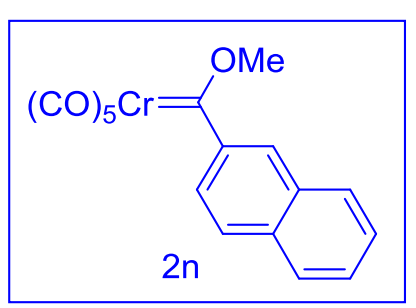

${ }^{1} \mathrm{H}$ NMR $\left(400 \mathrm{MHz}, \mathrm{CDCl}_{3}\right) \delta$ 7.93-7.84 (m, 4H), 7.55-7.46 (m, $3 \mathrm{H}), 4.77(\mathrm{~s}, 3 \mathrm{H}) .{ }^{13} \mathrm{C} \mathrm{NMR}\left(100 \mathrm{MHz}, \mathrm{CDCl}_{3}\right) \delta 349.45,224.10$, $216.26,150.79,133.93,132.25,129.58,127.95,127.86,127.71$, $127.02,124.05,121.03,67.25$.

Pentacarbonyl[(ethoxy)(phenyl)carbene $]$ chromium $(0)(20)^{13}$

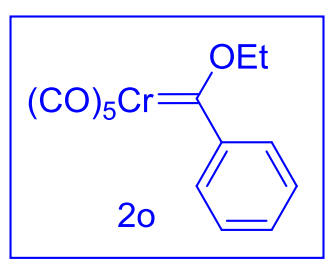

${ }^{1} \mathrm{H}$ NMR (400 MHz, $\left.\mathrm{CDCl}_{3}\right) \delta$ 7.39-7.25 (m, 5H), $4.94(\mathrm{~s}, 2 \mathrm{H}), 1.66(\mathrm{~s}$, $3 \mathrm{H}) .{ }^{13} \mathrm{C}$ NMR $\left(100 \mathrm{MHz}, \mathrm{CDCl}_{3}\right) \delta 349.05,224.23,216.21,153.79$, 130.06, 128.14, 122.73, 77.06, 15.16. 


\section{References}

(1) Cho, S. H.; Kim, J. Y.; Lee, S. Y.; Chang, S. Angew. Chem. Int. Ed. 2009, 48, 9127-9130.

(2) (a) Sierra, M. A.; Mancheño, M. J.; Sáez, E.; del Amo, J. C. J. Am. Chem. Soc. 1998, 120, 6812-6813. (b) Sierra, M. A.; del Amo, J. C.; Mancheño, M. J.; Gómez-Gallego, M. J. Am. Chem. Soc. 2001, 123, 851-861. (c) Chu, G. M.; Fernández, I.; Sierra, M. A. J. Org. Chem. 2013, 78, 865-871.

(3) Froehr, T.; Sindlinger, C. P.; Kloeckner, U.; Finkbeiner, P.; Nachtsheim, B. J. Org. Lett. 2011, 13, 3754-3757.

(4) Ackermann, L.; Barfüsser, S.; Pospech, J. Org. Lett. 2010, 12, 724-726.

(5) Wertz, S., Kodama, S.; Studer, A. Angew. Chem. Int. Ed. 2011, 50, 11511-11515.

(6) Zhao, X., Ding, F.; Li, J.; Lu, K.; Lu, X.; Wang, B.; Yu, P. Tetrahedron Lett. 2015, 56, 511-513.

(7) Wang, G.-W., Zhou, A.-X.; Wang, J.-J.; Hu, R.-B.; Yang, S.-D. Org. Lett. 2013, 15, 5270-5273.

(8) Bos, M. E.; Wulff, W. D.; Miller, R. A.; Chamberlin, S.; Brandvold, T. A. J. Am. Chem. Soc. 1991, 113, 9293-9319.

(9) Dötz, K. H.; Erben, H.-G.; Staudacher, W.; Harms, K. J. Organomet. Chem. 1988, 355, 177-191.

(10) Bernasconi, C. F.; García-Río, L. J. Am. Chem. Soc. 2000, 122, 3821-3829.

(11) Barluenga, J.; Pérez-Sánchez, I.; Suero, M. G.; Rubio, E.; Flórez, J. Chem. Eur. J. 2006, $12,7225-7235$.

(12) Suero, M. G.; De la Campa, R.; Torre-Fernández, L.; García-Granda, S.; Flórez, J. Chem. Eur. J. 2012, 18, 7287-7295.

(13) Bernasconi, C. F.; Kittredge, K. W.; Flores, F. X. J. Am. Chem. Soc. 1999, 121, 6630-6639.

\section{Experiments for mechanistic studies}

Scheme S1. Benzoxazole competition experiment

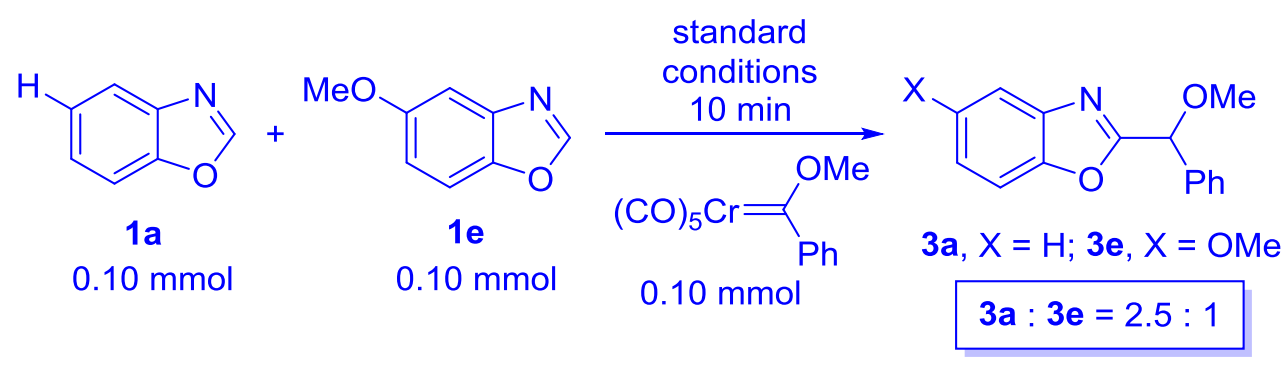

Benzoxazole 1a (11.9 mg, $0.10 \mathrm{mmol})$, 5-methoxybenzoxazole 1e (14.9 mg, $0.10 \mathrm{mmol})$, chromium carbene complexe $\mathbf{2 a}(31.2 \mathrm{mg}, 0.10 \mathrm{mmol})$ and lithium tert-butoxide $\left(\mathrm{LiO}^{t} \mathrm{Bu}, 16\right.$ $\mathrm{mg}, 0.20 \mathrm{mmol}$ ) were successively added to a $10 \mathrm{~mL}$ Schlenk reaction tube. The reaction tube was then degassed for two times with nitrogen and toluene $(1 \mathrm{~mL})$ was added using a syringe. The resulting solution was stirred at $100{ }^{\circ} \mathrm{C}$ for the $10 \mathrm{~min}$. The mixture was then cooled to room temperature and filtered through a short plug of silica gel (washed with petroleum 
ether:EtOAc = 3:1). Solvent was then removed in vacuo to provide a crude mixture, which was detected by ${ }^{1} \mathrm{H}$ NMR analysis using mesitylene as the internal standard (Scheme S1, Figure S1).

Figure S1. ${ }^{1} \mathrm{H}$ NMR spectrum of the crude product of benzoxazole competition experiment

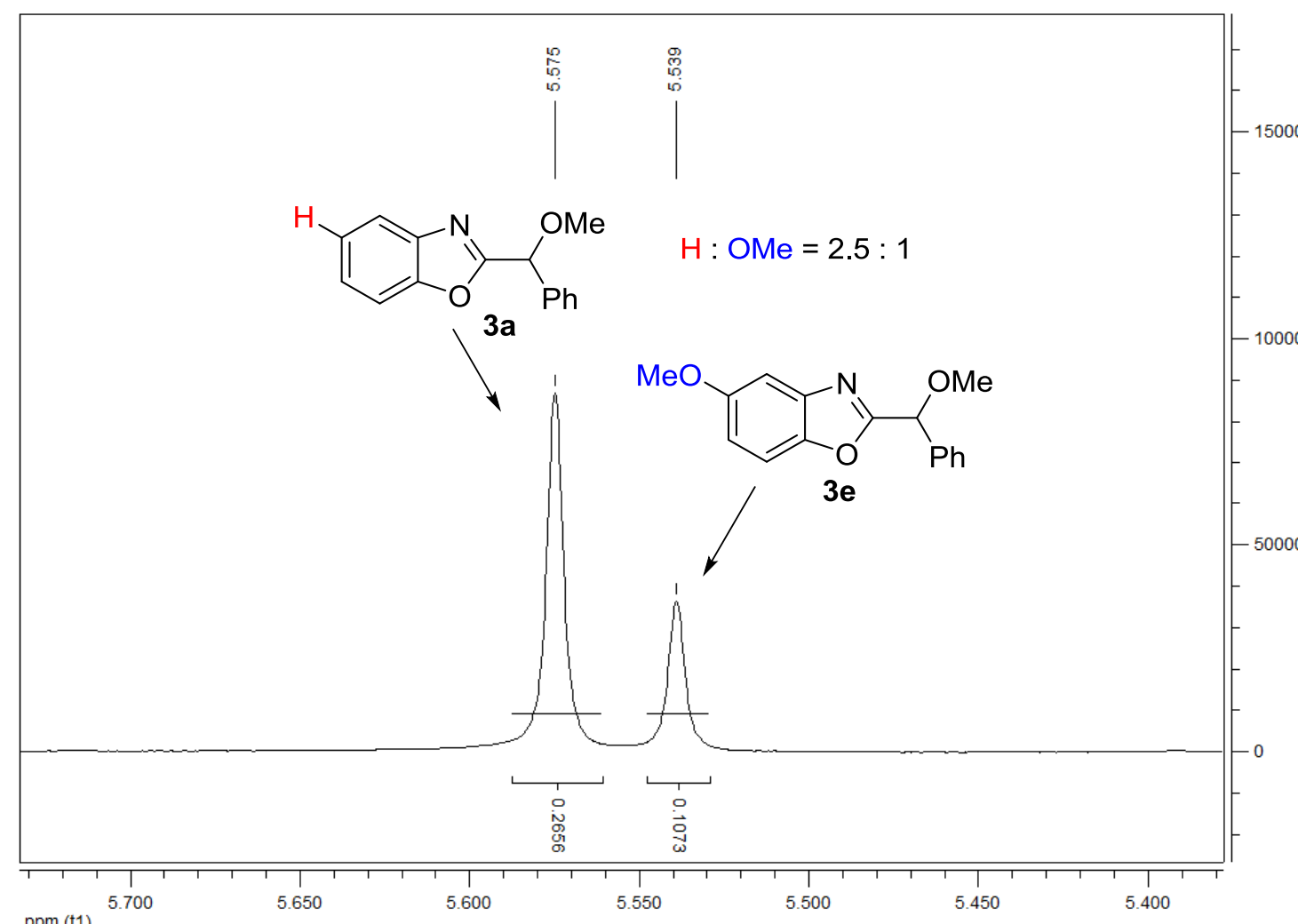

Scheme S2. Chromium carbene competition experiment<smiles>COC(=C(C)C(C)(C)C)c1ccc(F)cc1</smiles>

$0.05 \mathrm{mmol}$<smiles>COC(=C(C)C(C)(C)C)c1ccc(OC)cc1</smiles>

$0.05 \mathrm{mmol}$

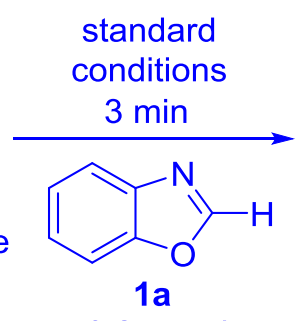

$0.2 \mathrm{mmol}$<smiles>[X]c1ccc(C(OC)c2nc3ccccc3o2)cc1</smiles>

4f, $X=O M e ; ~ 4 h, X=F$

$\mathbf{4 f}: \mathbf{4 h}=1: 1.5$

Benzoxazole 1a $(23.8 \mathrm{mg}, 0.20 \mathrm{mmol})$, chromium carbene complexe $2 \mathbf{i}$ (16.5mg, 0.05 mmol), chromium carbene complex $2 \mathrm{~g}(17.1 \mathrm{mg}, 0.05 \mathrm{mmol})$ and lithium tert-butoxide $\left(\mathrm{LiO}^{t} \mathrm{Bu}, 16 \mathrm{mg}, 0.20 \mathrm{mmol}\right.$ ) were successively added to a $10 \mathrm{~mL}$ Schlenk reaction tube. The reaction tube was then degassed for two times with nitrogen and toluene $(1 \mathrm{~mL})$ was added using a syringe. The resulting solution was stirred at $100{ }^{\circ} \mathrm{C}$ for the $3 \mathrm{~min}$. The mixture was then cooled to room temperature and filtered through a short plug of silica gel (washed with petroleum ether:EtOAc $=3: 1$ ). Solvent was then removed in vacuo to provide a crude mixture, 
which was detected by ${ }^{1} \mathrm{H}$ NMR analysis using mesitylene as the internal standard (Scheme S2, Figure S2).

Figure S2. ${ }^{1} \mathrm{H}$ NMR spectrum of the crude product of chromium carbene competition experiment

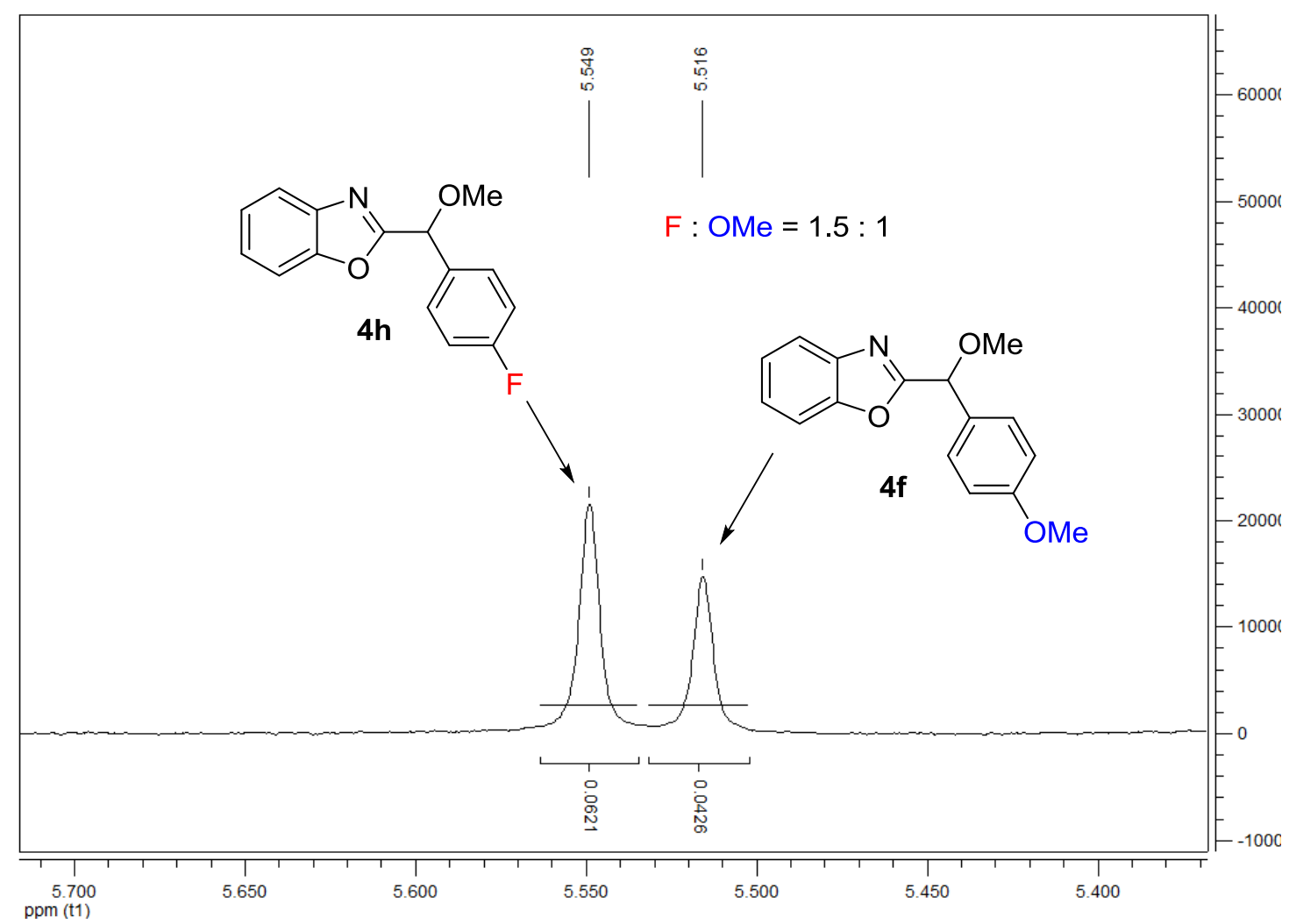




\section{4. ${ }^{1} \mathrm{H}$ and ${ }^{13} \mathrm{C}$ NMR spectra of substrates and products}

Figure S3. ${ }^{1} \mathrm{H}$ NMR of 4-methylbenzo[d]oxazole (1b)

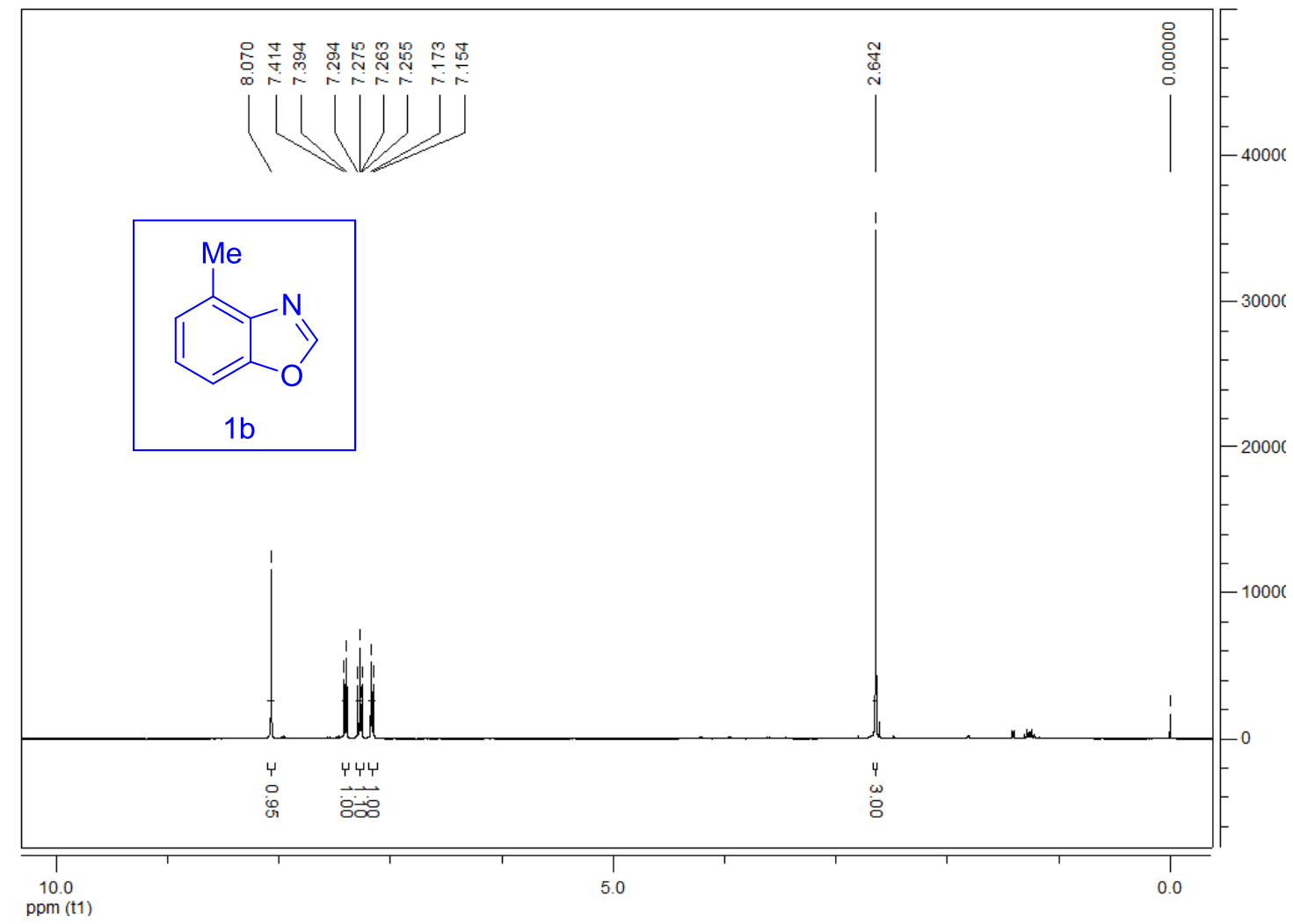

Figure S4. ${ }^{13} \mathrm{C}$ NMR of 4-methylbenzo[d]oxazole (1b)

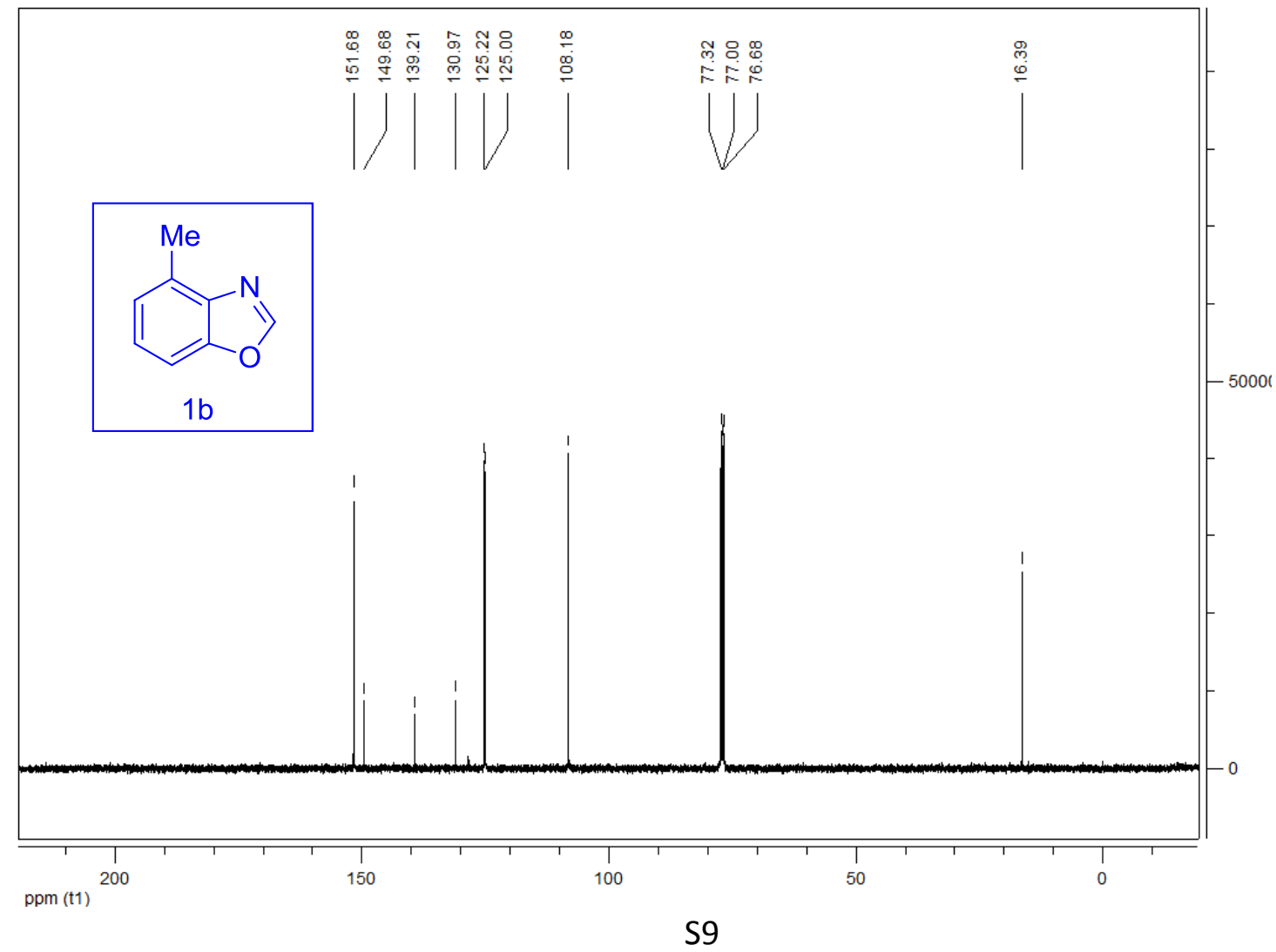


Figure S5. ${ }^{1} \mathrm{H}$ NMR of 5-(tert-butyl)benzo[d]oxazole (1d)

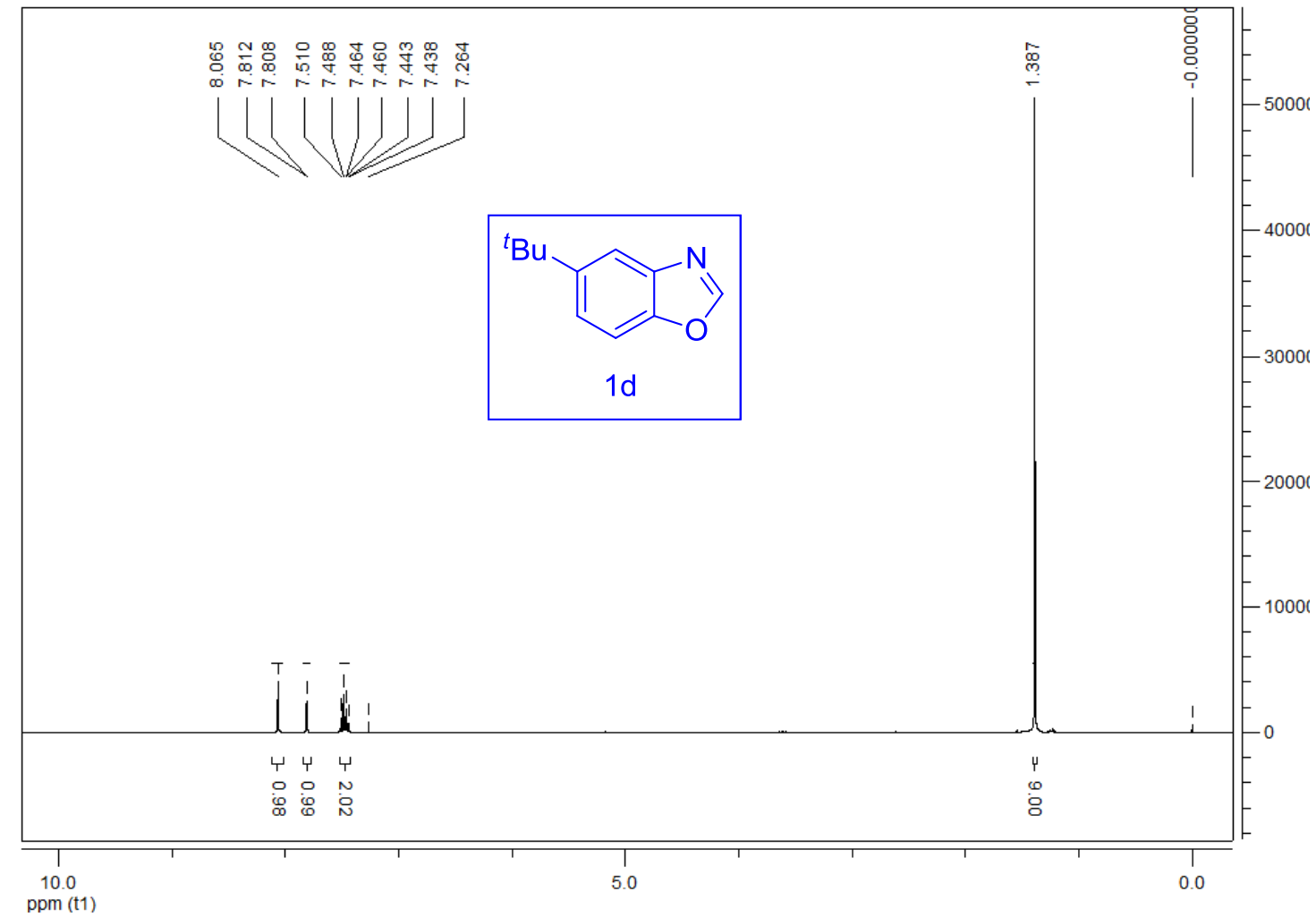

Figure S6. ${ }^{13} \mathrm{C}$ NMR of 5-(tert-butyl)benzo[d]oxazole (1d)

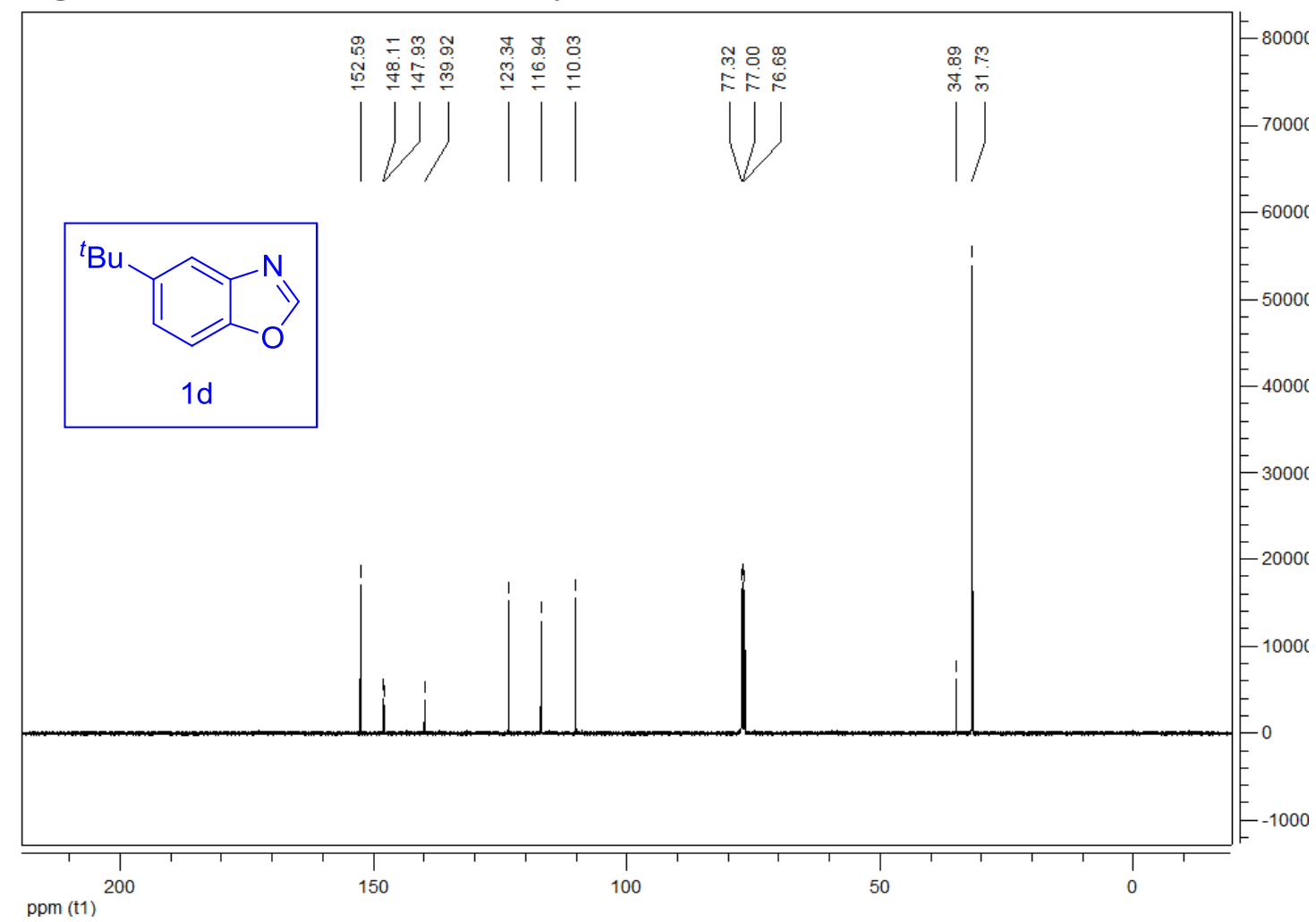




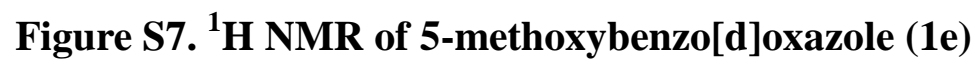

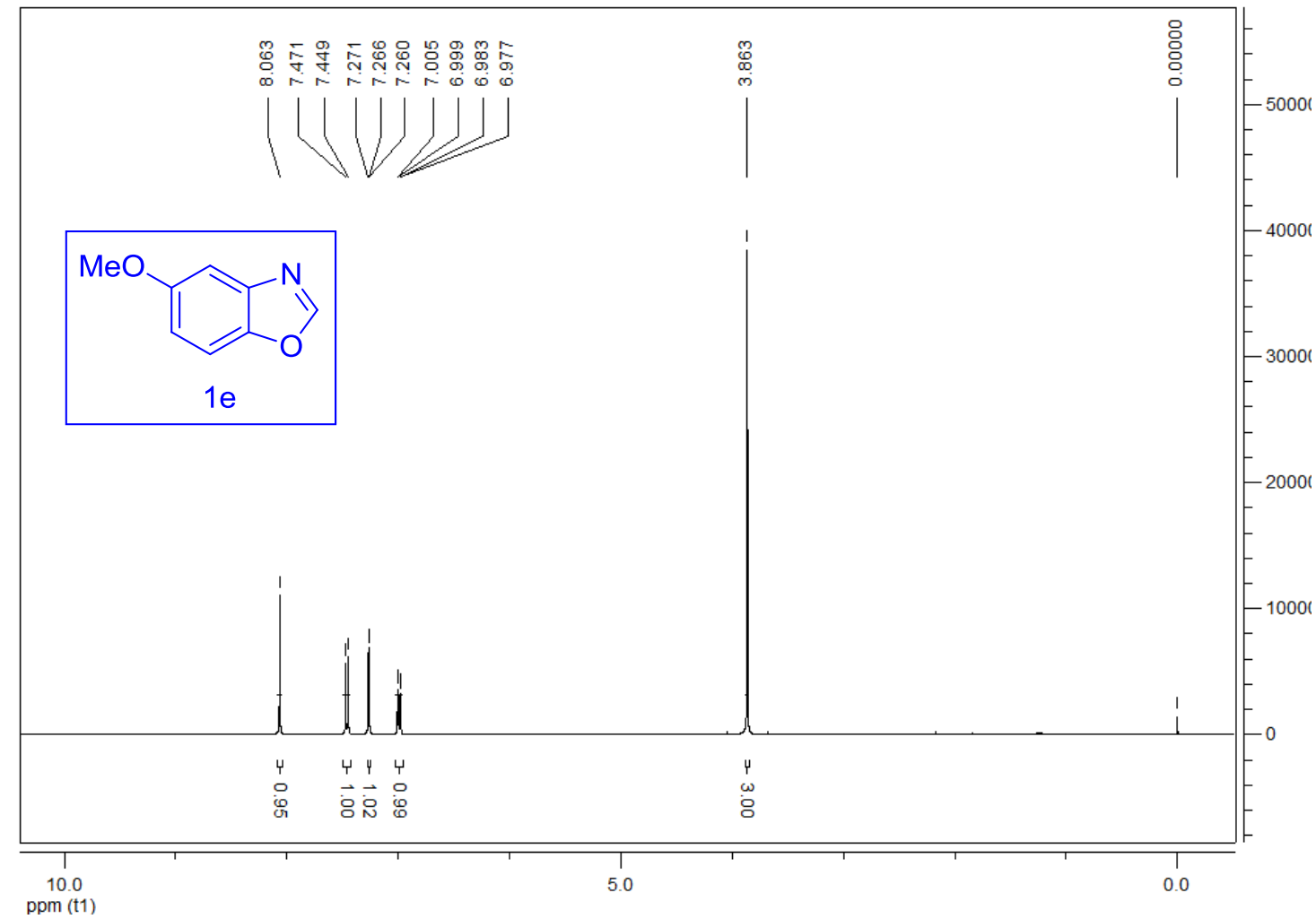

Figure S8. ${ }^{13} \mathrm{C}$ NMR of 5-methoxybenzo[d]oxazole (1e)

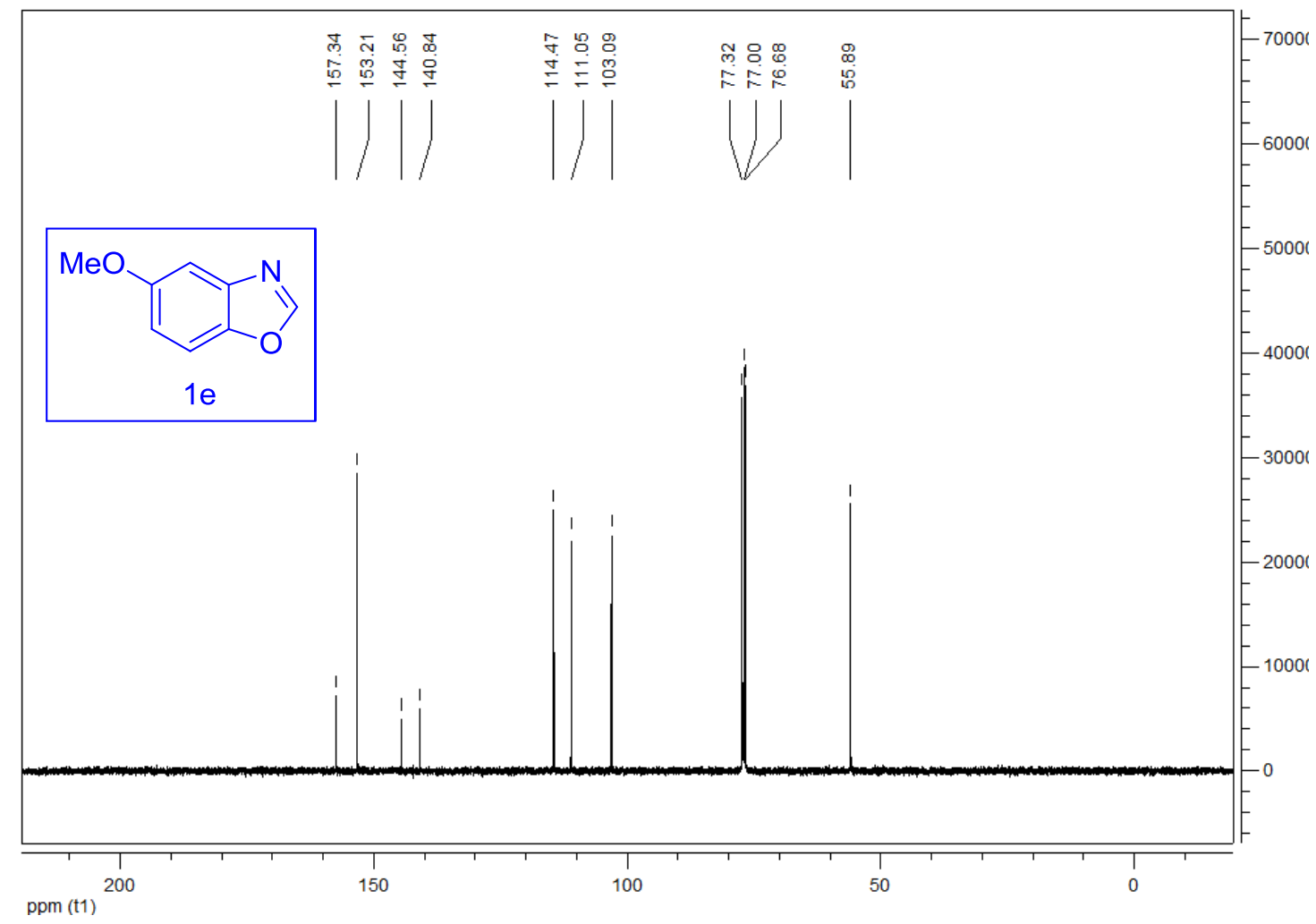


Figure S9. ${ }^{1} \mathrm{H}$ NMR of 5 -fluorobenzo[d]oxazole (1f)

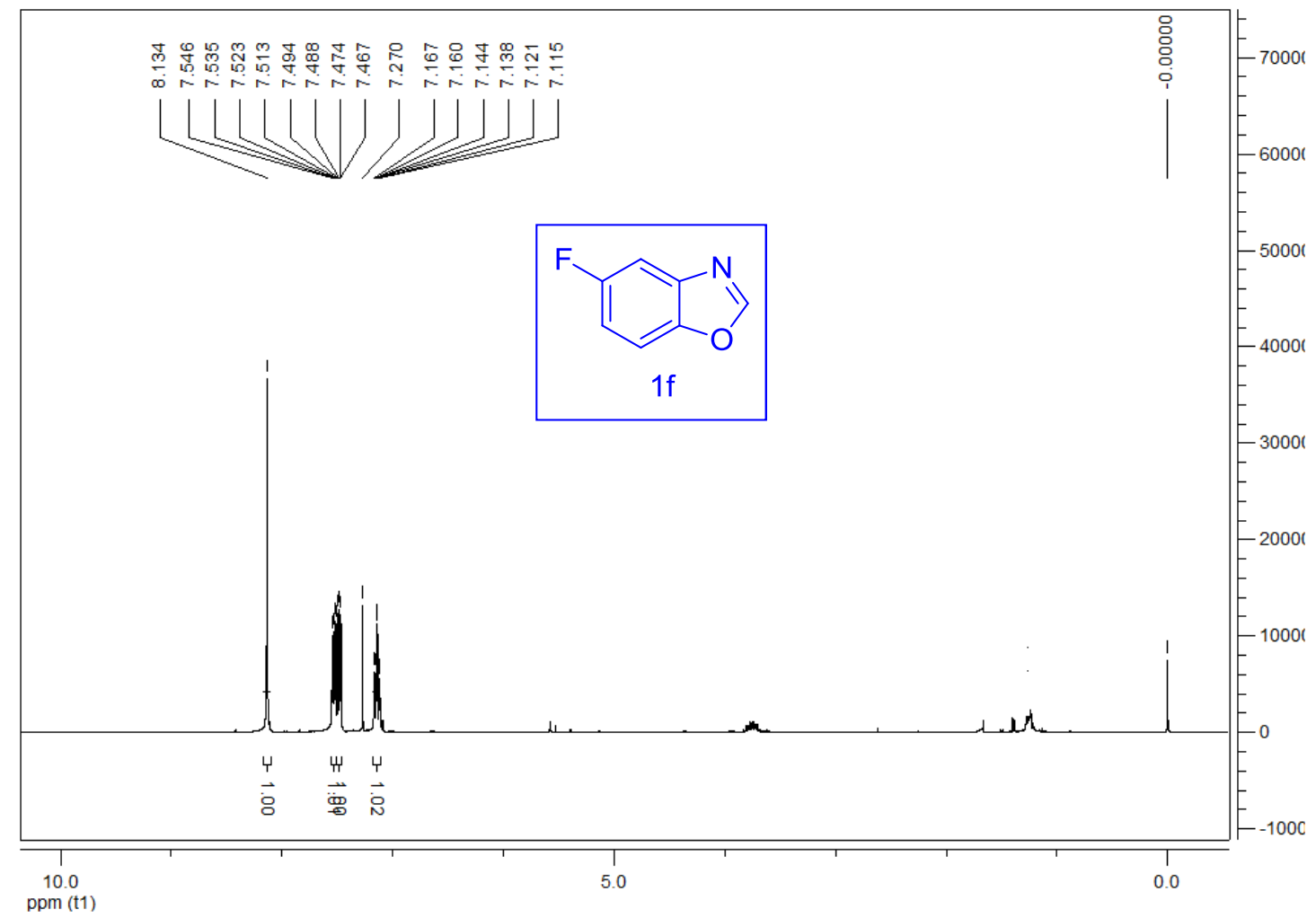

Figure S10. ${ }^{13} \mathrm{C}$ NMR of 5-fluorobenzo[d]oxazole (1f)

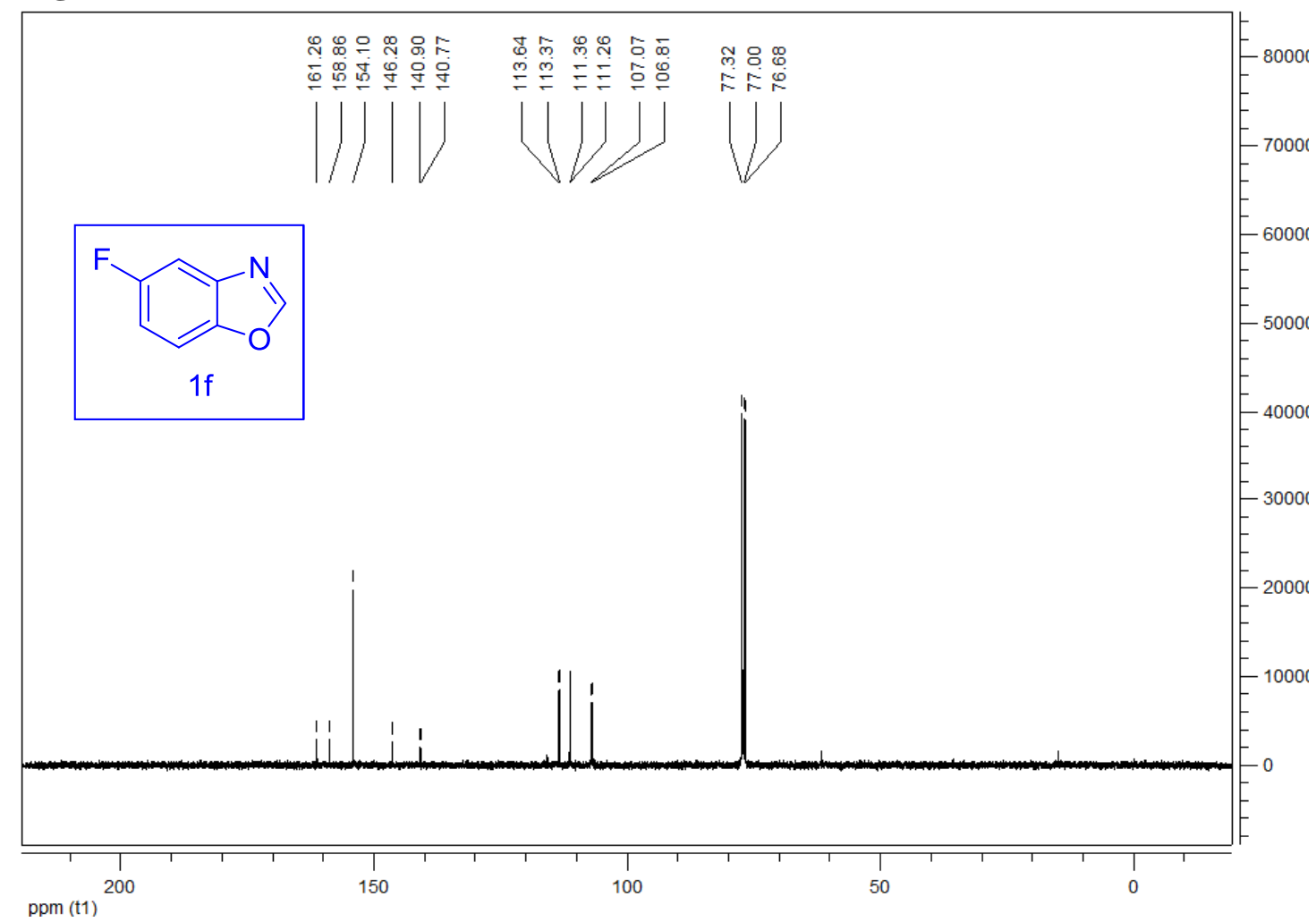


Figure S11. ${ }^{1} \mathrm{H}$ NMR of 6-methylbenzo[d]oxazole (1h)

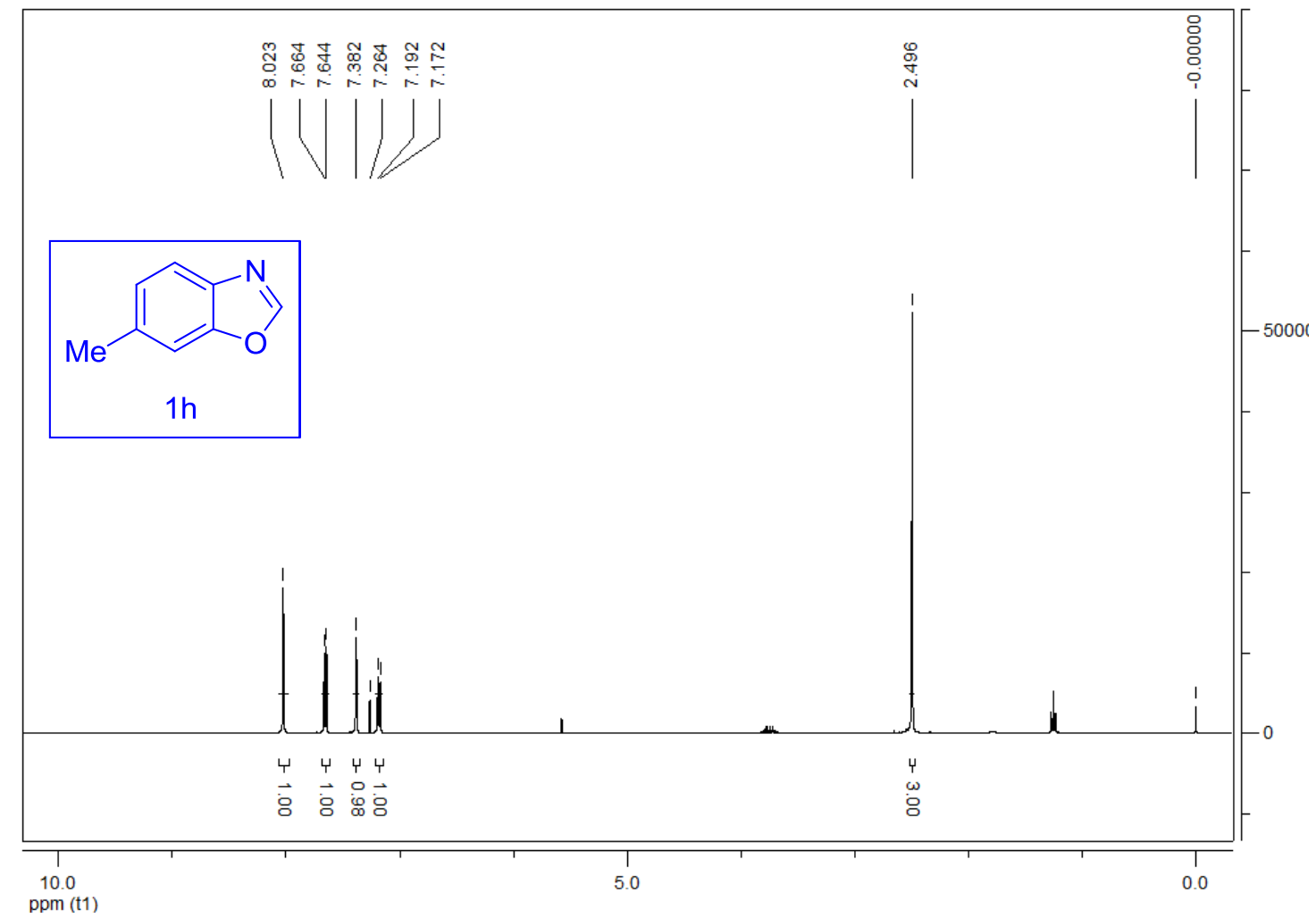

Figure S12. ${ }^{13} \mathrm{C}$ NMR of 6-methylbenzo[d]oxazole (1h)

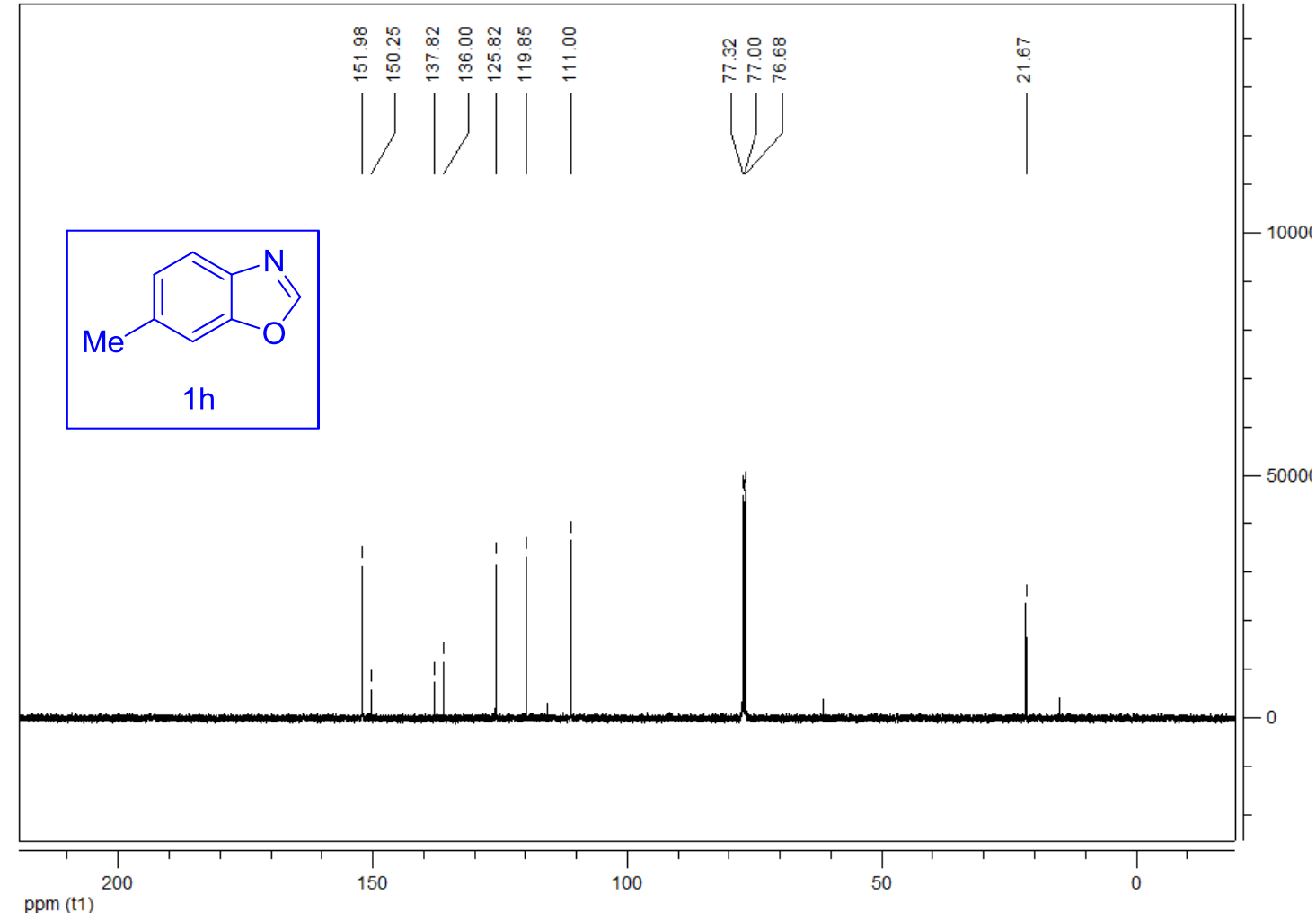


Figure S13. ${ }^{1} \mathrm{H}$ NMR of 6-chlorobenzo[d]oxazole (1i)

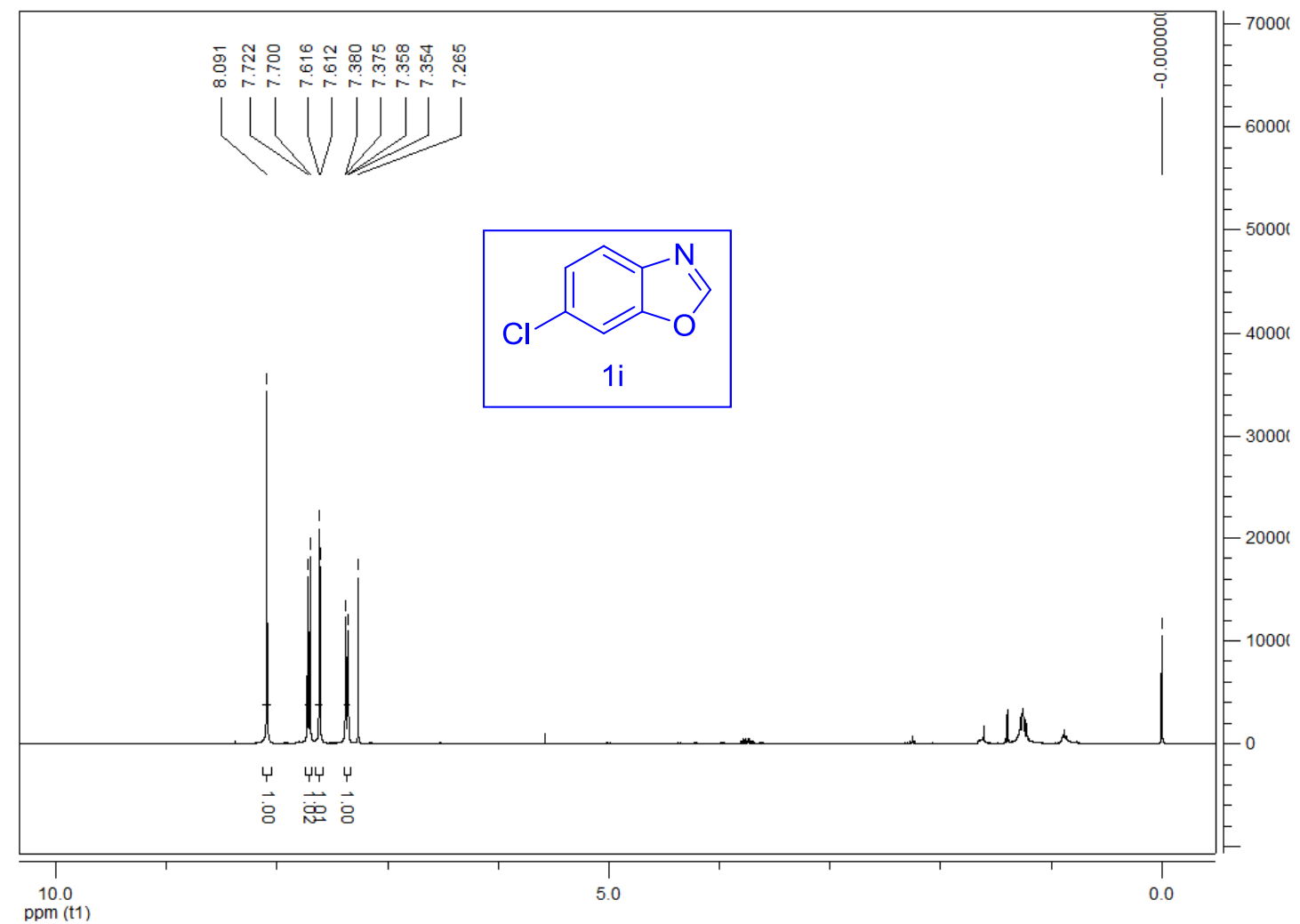

Figure S14. ${ }^{13} \mathrm{C}$ NMR of 6-chlorobenzo[d]oxazole (1i)

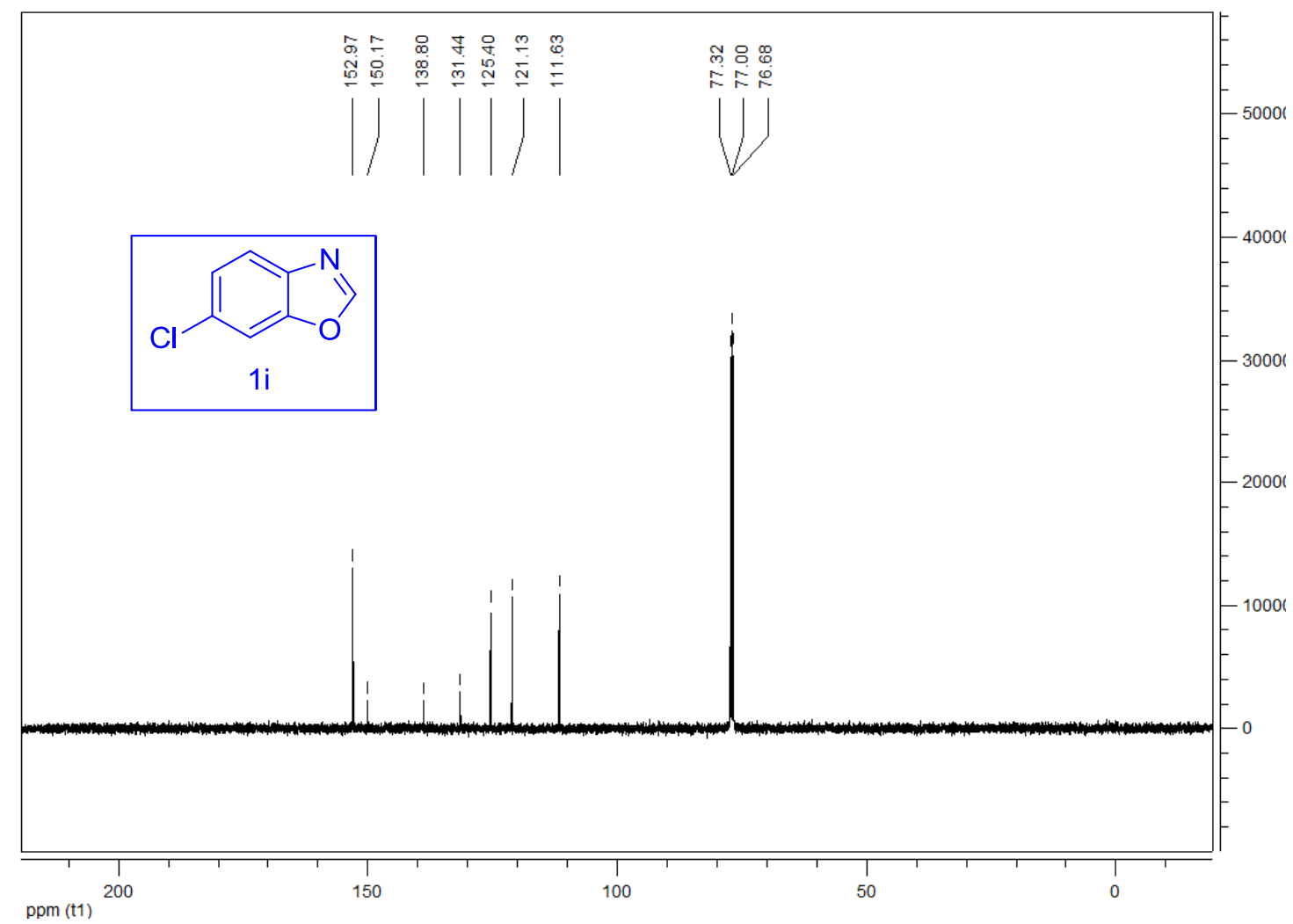


Figure S15. ${ }^{1} \mathrm{H}$ NMR of 7-methylbenzo[d]oxazole (1j)

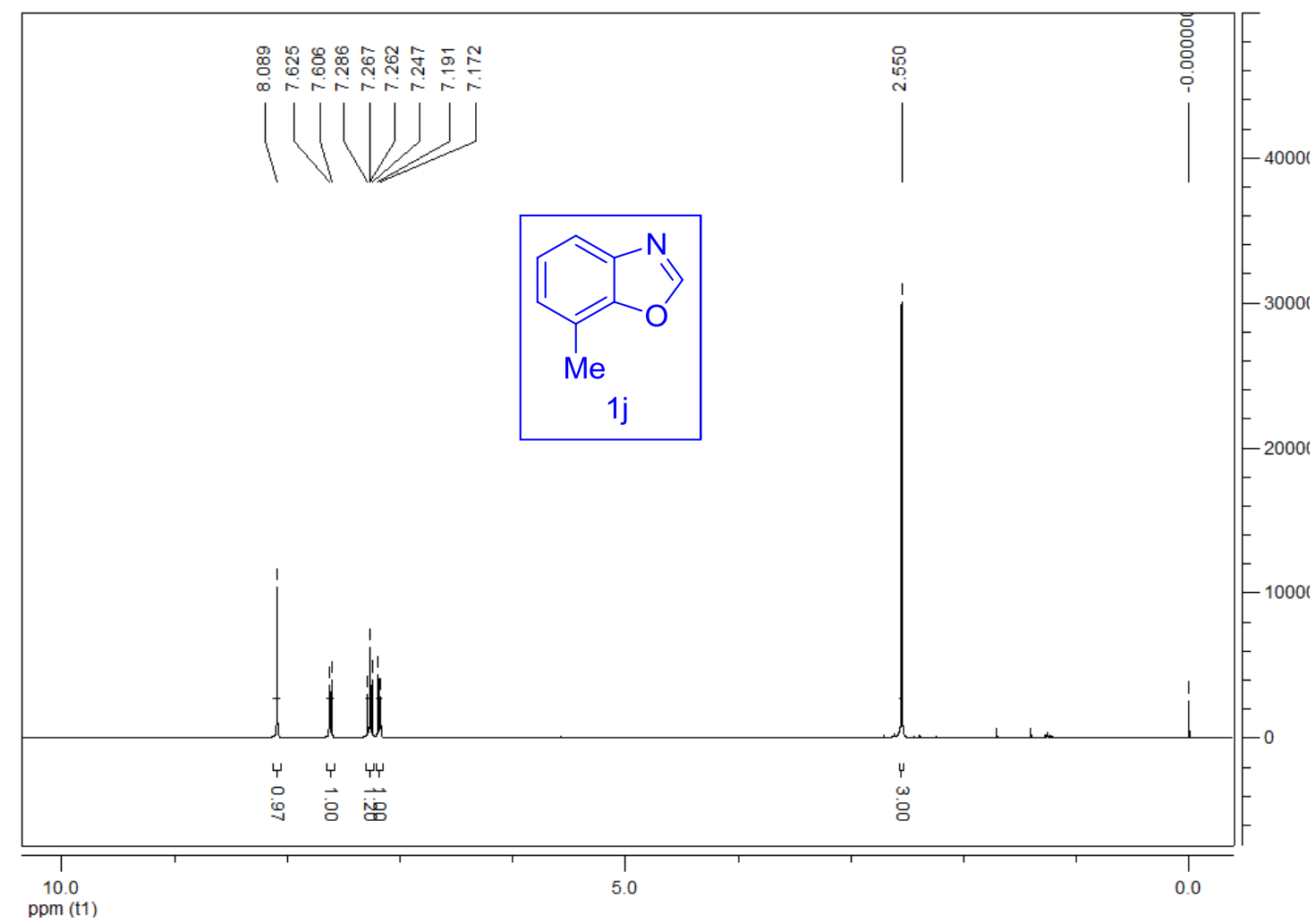

Figure S16. ${ }^{13} \mathrm{C}$ NMR of 7-methylbenzo[d]oxazole (1j)

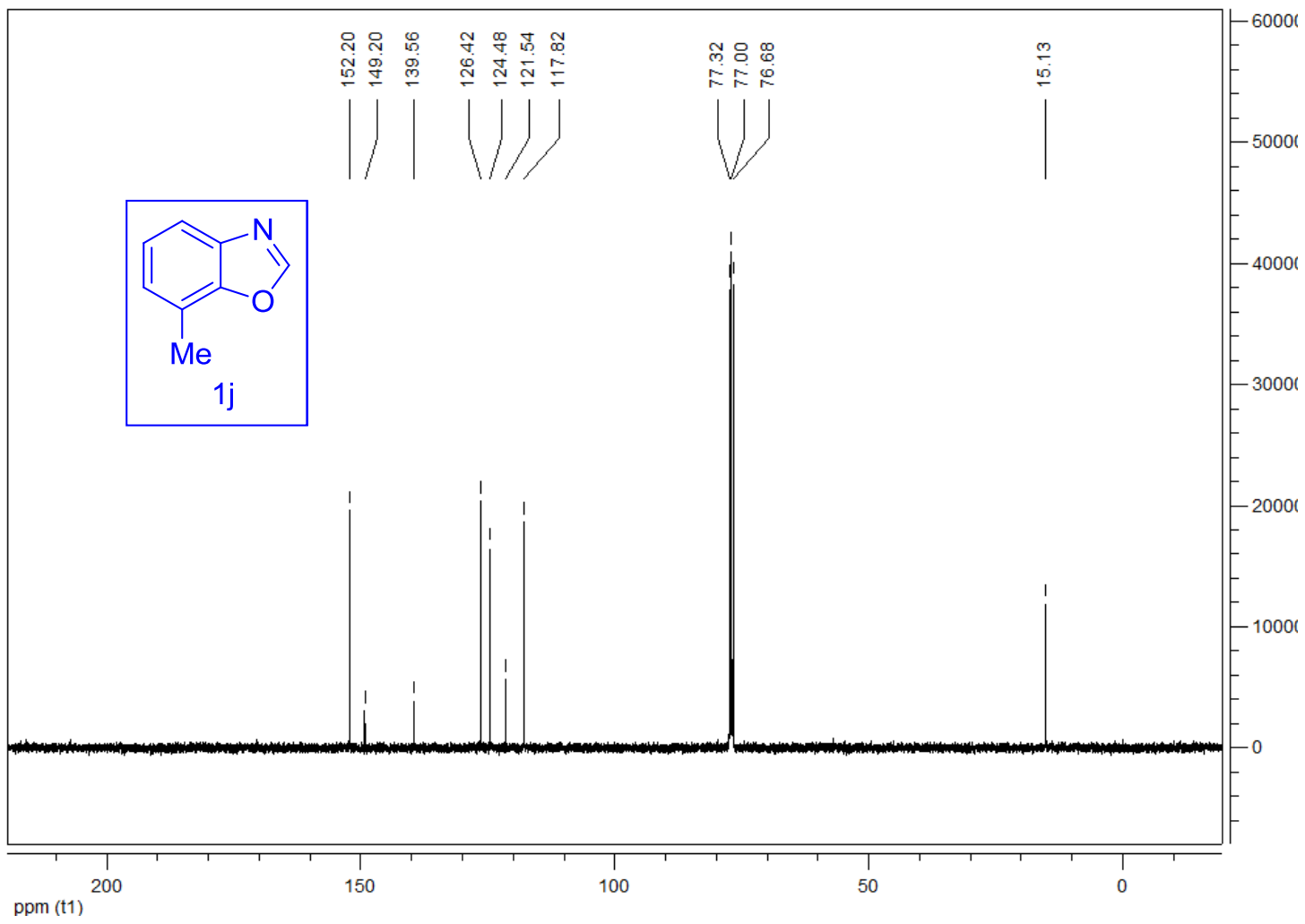


Figure S17. ${ }^{1} \mathrm{H}$ NMR of 7-bromobenzo[d]oxazole (1k)

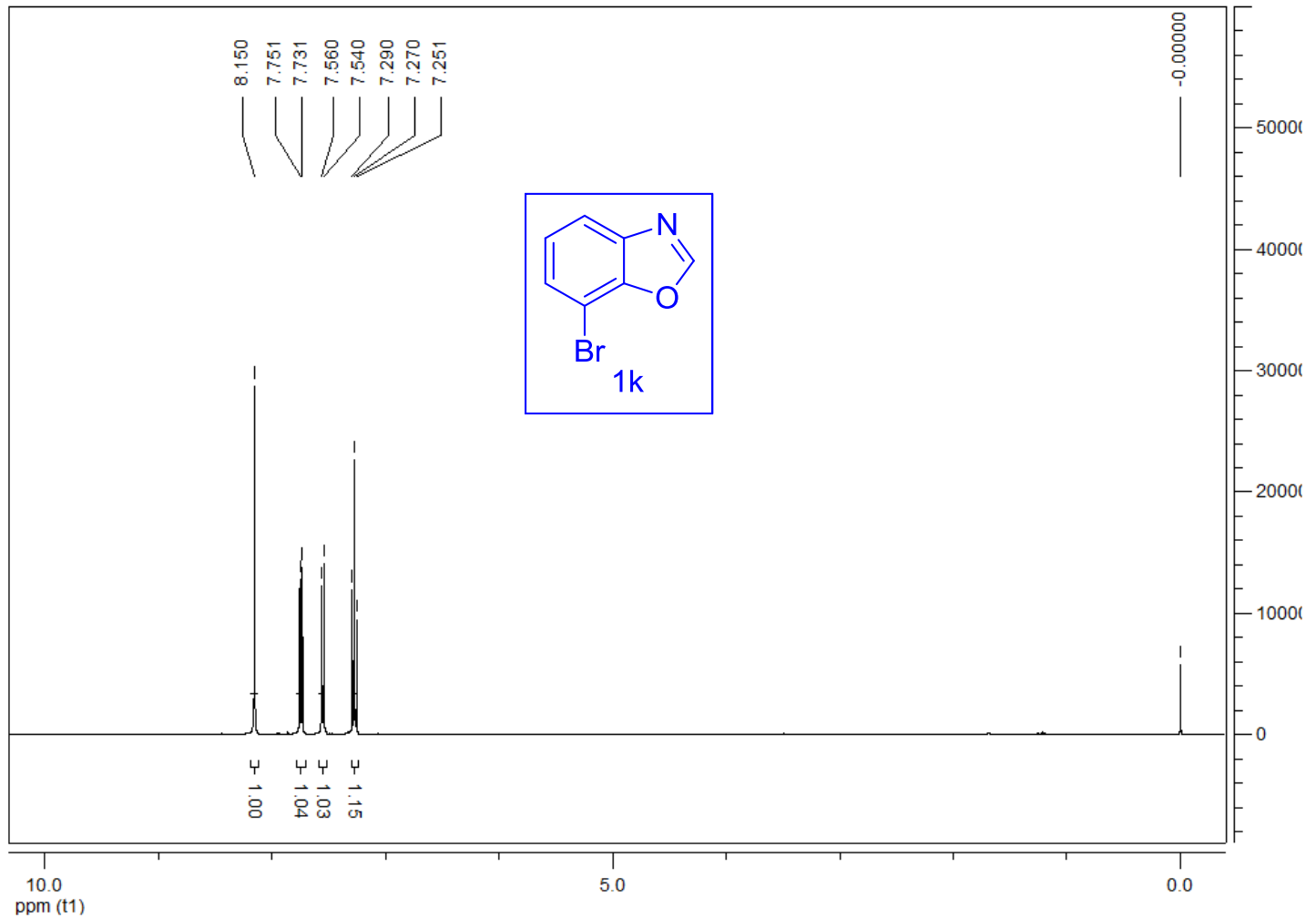

Figure S18. ${ }^{13} \mathrm{C}$ NMR of 7-bromobenzo[d]oxazole (1k)

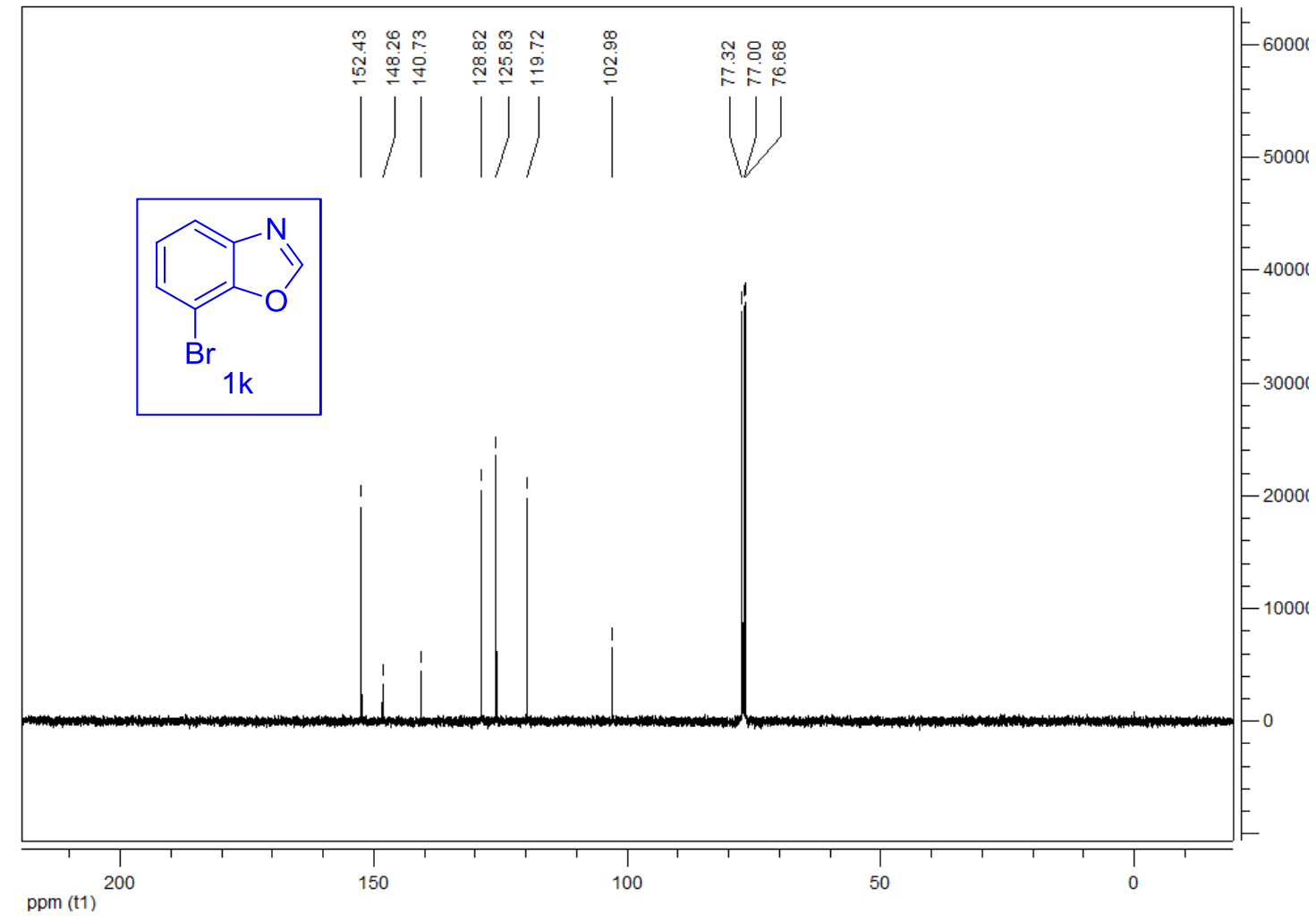


Figure S19. ${ }^{1} \mathrm{H}$ NMR of pentacarbonyl[(methoxy)(phenyl)carbene $] \operatorname{chromium}(0)(2 a)$

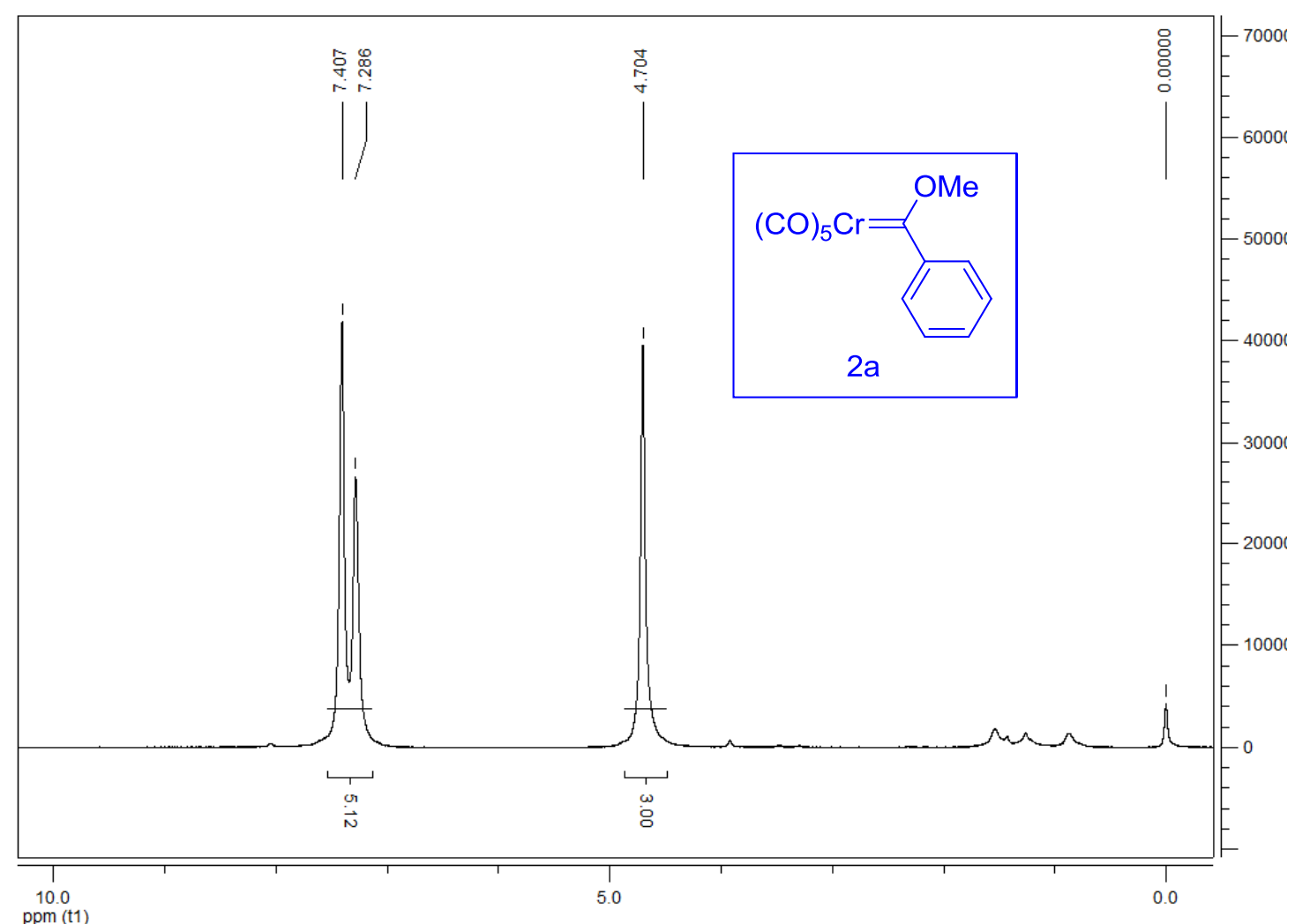

Figure S20. ${ }^{13} \mathrm{C}$ NMR of pentacarbonyl[(methoxy)(phenyl)carbene $] \operatorname{chromium}(0)(2 a)$

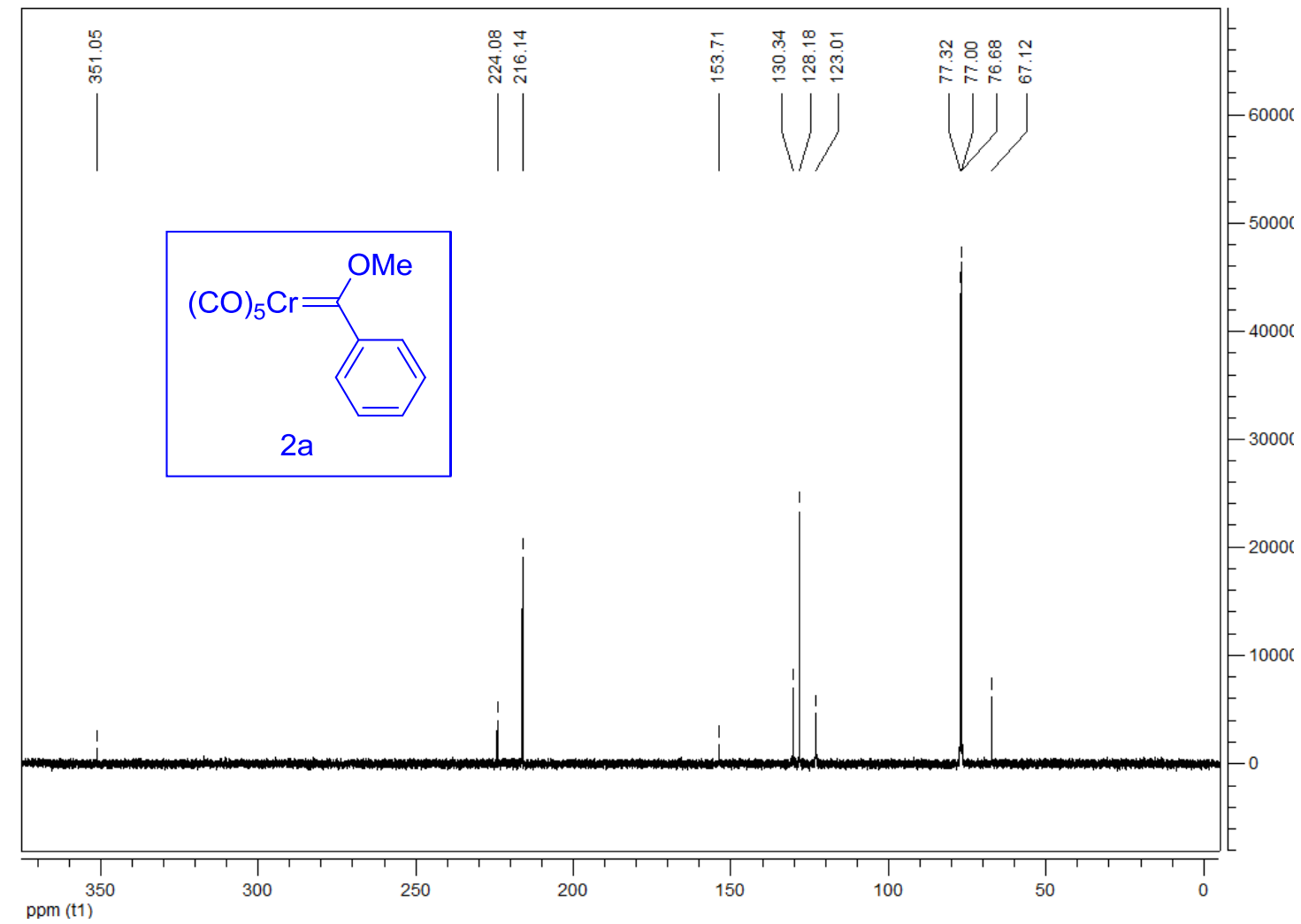


Figure S21. ${ }^{1} \mathrm{H}$ NMR of pentacarbonyl[(2-methylphenyl)(methoxy)carbene $]$ chromium(0) (2b)

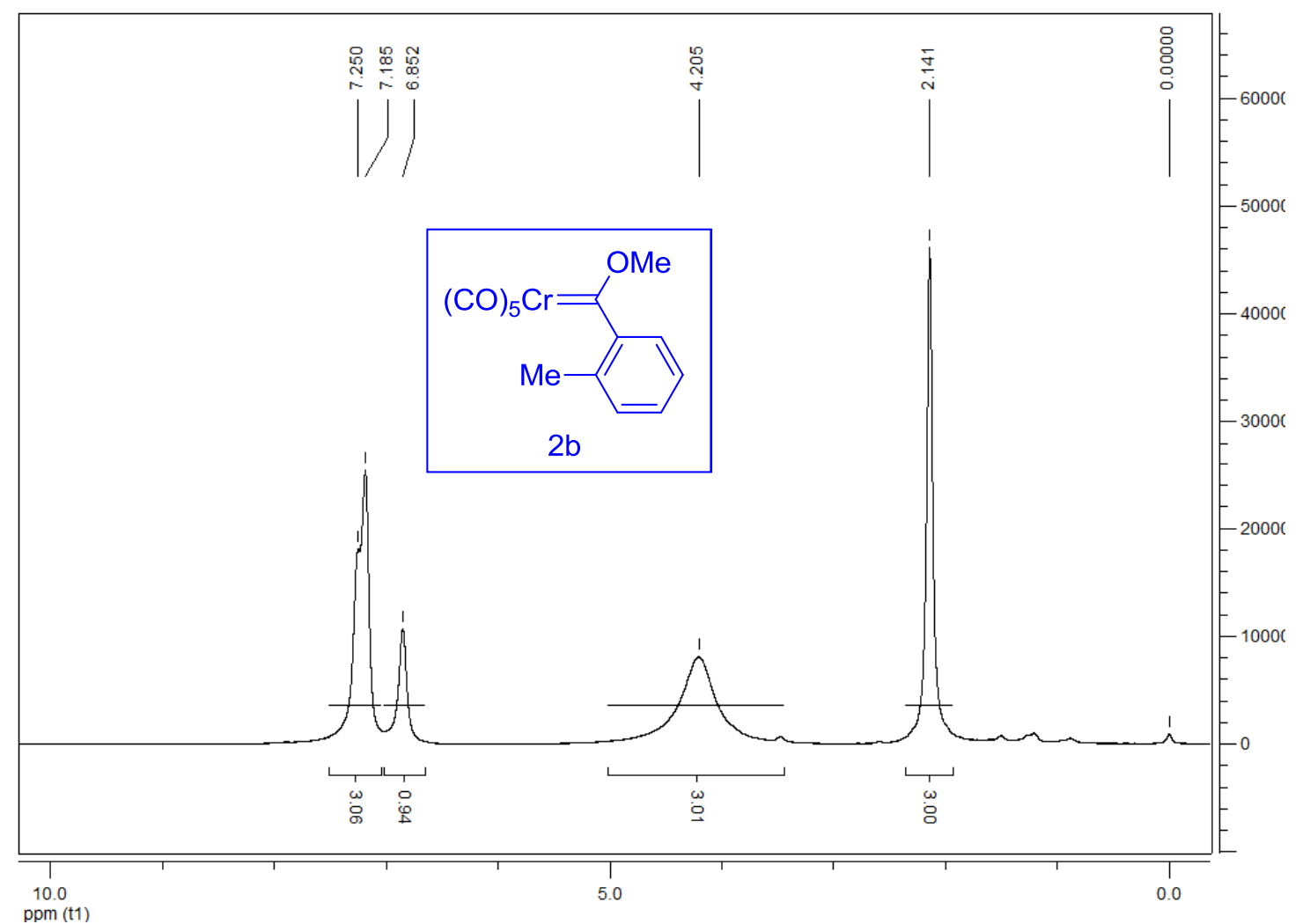

Figure S22. ${ }^{13} \mathrm{C}$ NMR of pentacarbonyl[(2-methylphenyl)(methoxy)carbene]chromium(0) (2b)

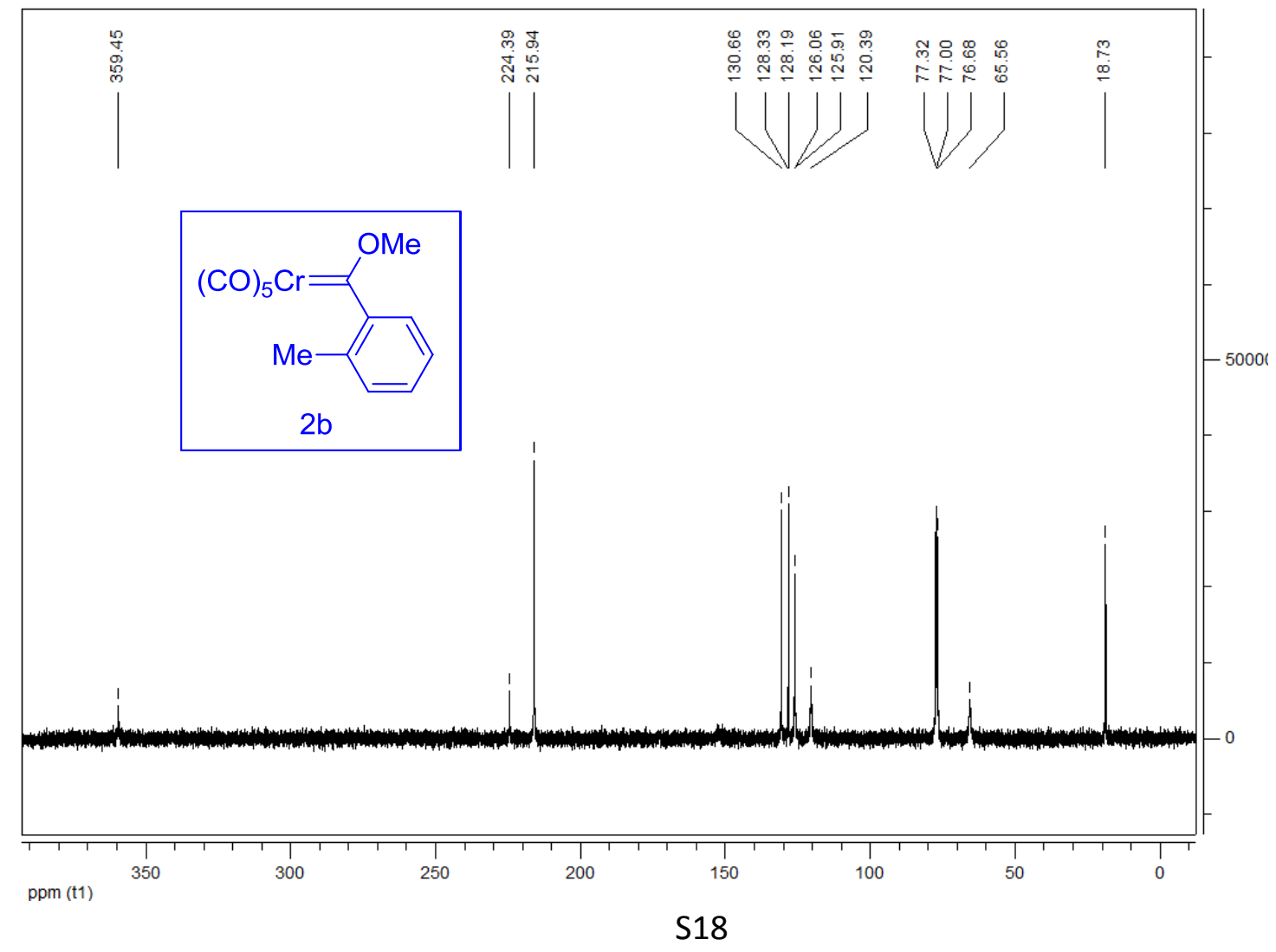


Figure S23. ${ }^{1}$ H NMR of pentacarbonyl[(methoxy)(2-methoxyphenyl)carbene]chromium (2c)

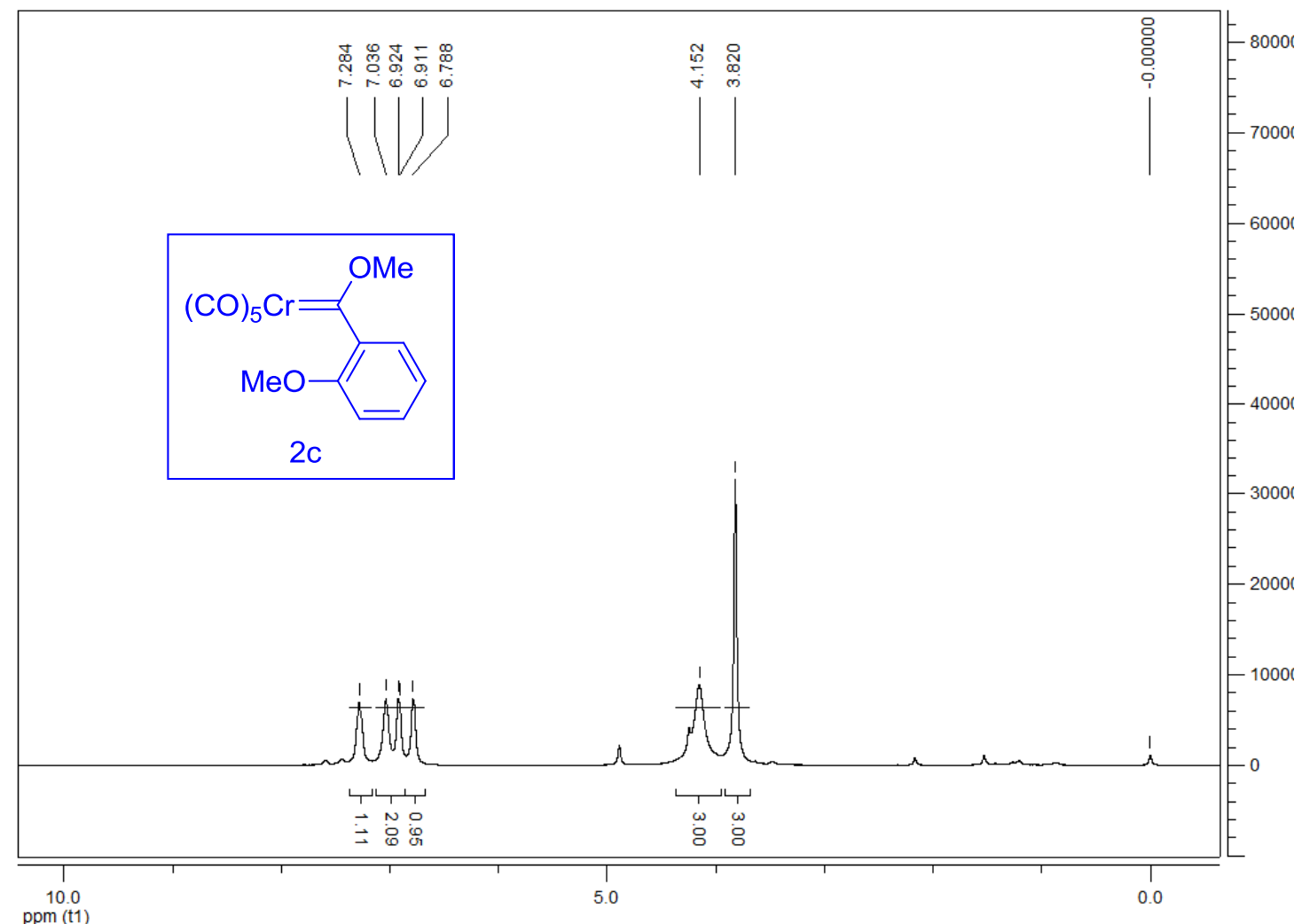

Figure S24. ${ }^{13} \mathrm{C}$ NMR of pentacarbonyl[(methoxy)(2-methoxyphenyl)carbene]chromium (2c)

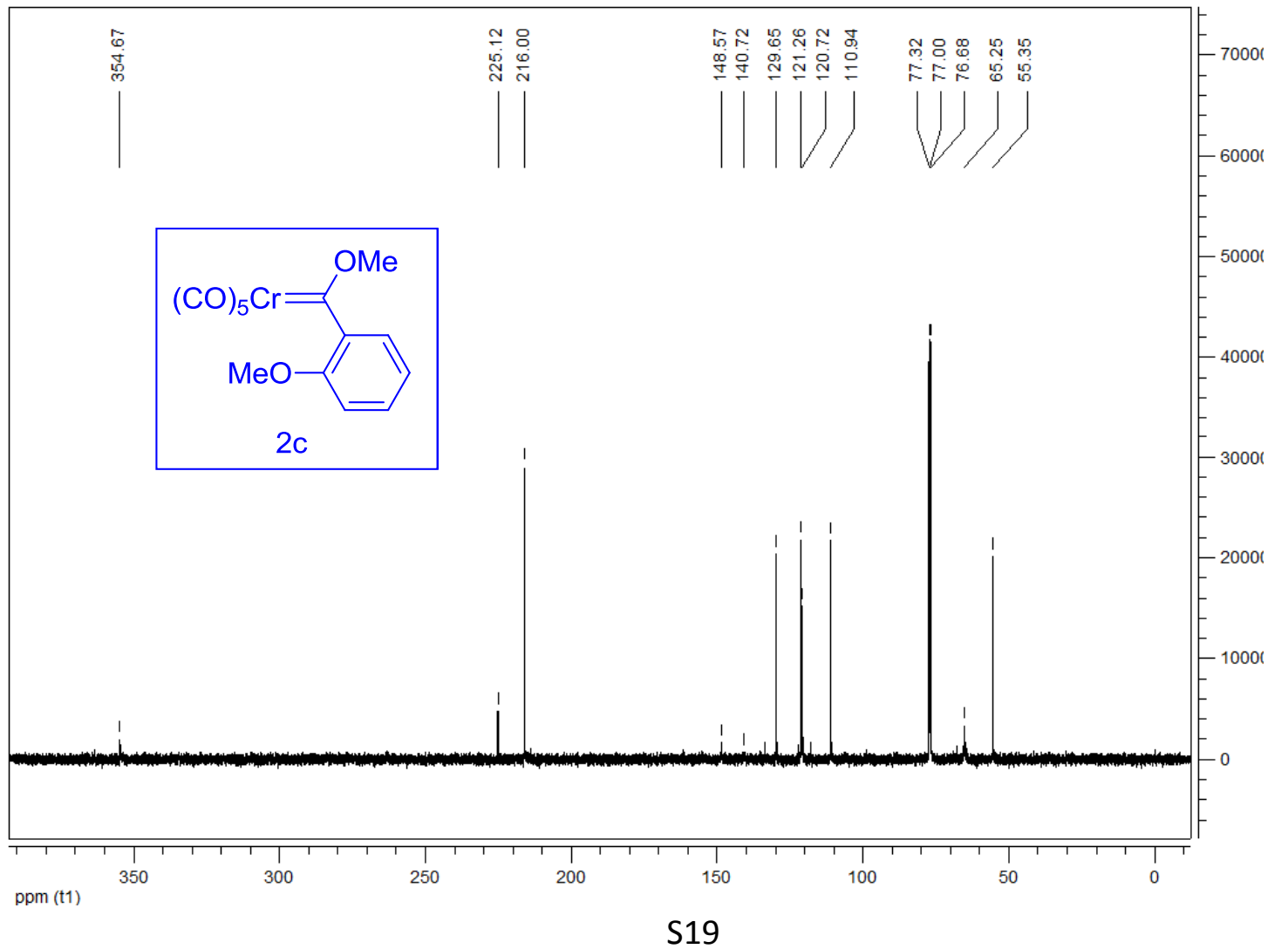


Figure S25. ${ }^{1} \mathrm{H}$ NMR of pentacarbonyl[(3-chlorophenyl)(methoxy)carbene $]$ chromium(0) (2d)

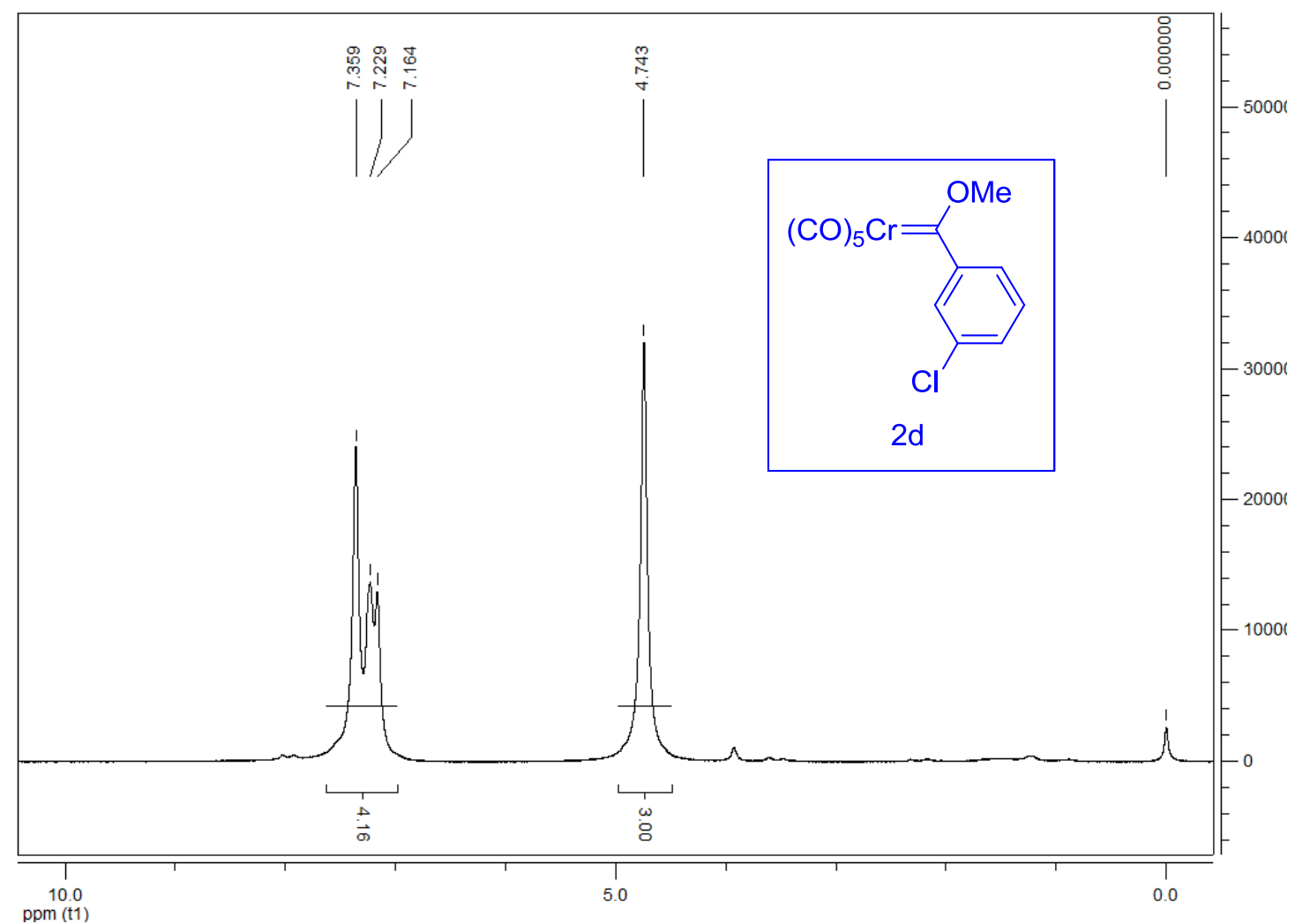

Figure S26. ${ }^{13} \mathrm{C}$ NMR of pentacarbonyl[(3-chlorophenyl)(methoxy)carbene $]$ chromium(0) (2d)

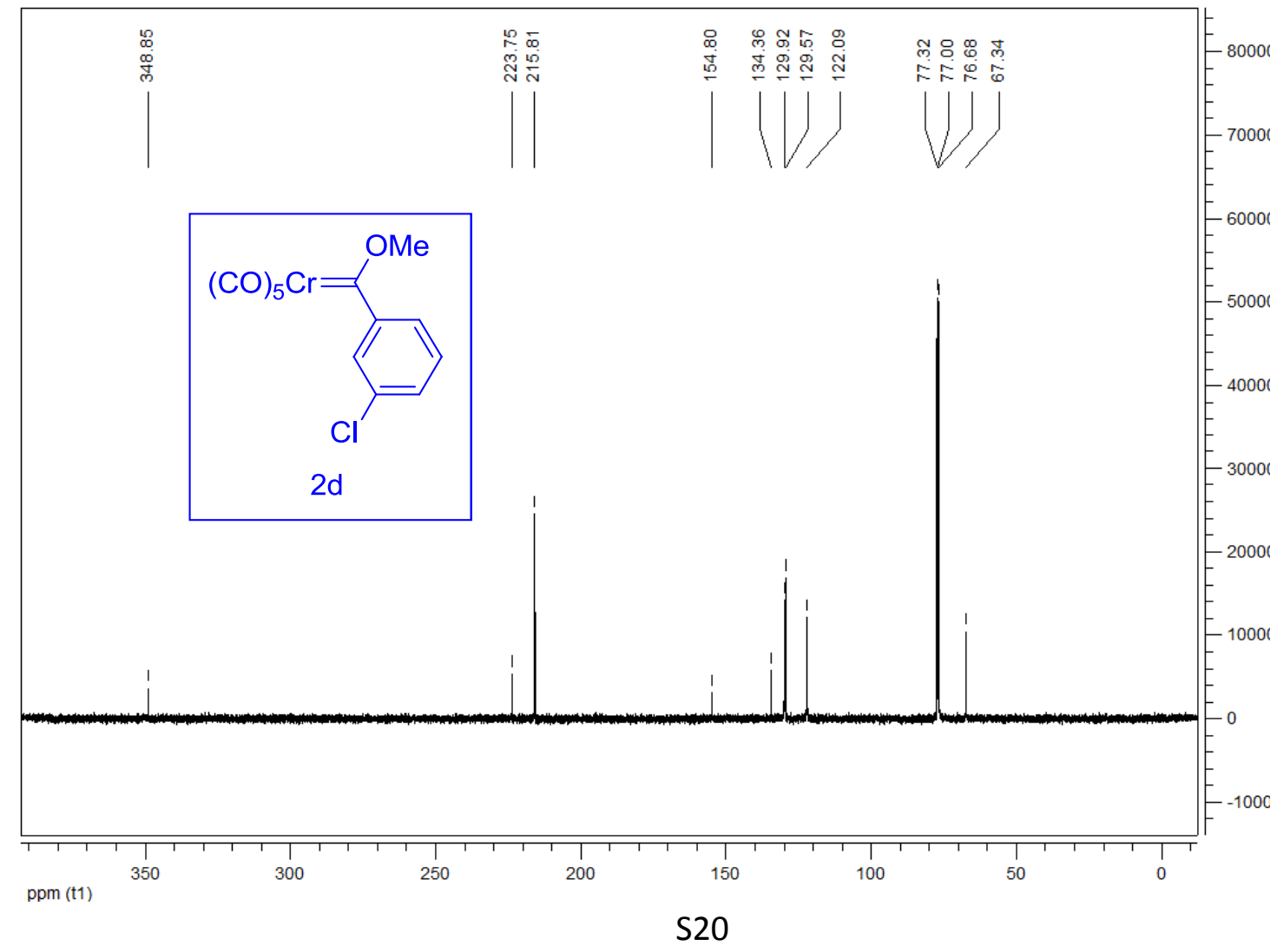


Figure S27. ${ }^{1}$ H NMR of pentacarbonyl[(methoxy)(3-methoxyphenyl)carbene] $\operatorname{chromium}(\mathbf{0})(2 \mathrm{e})$

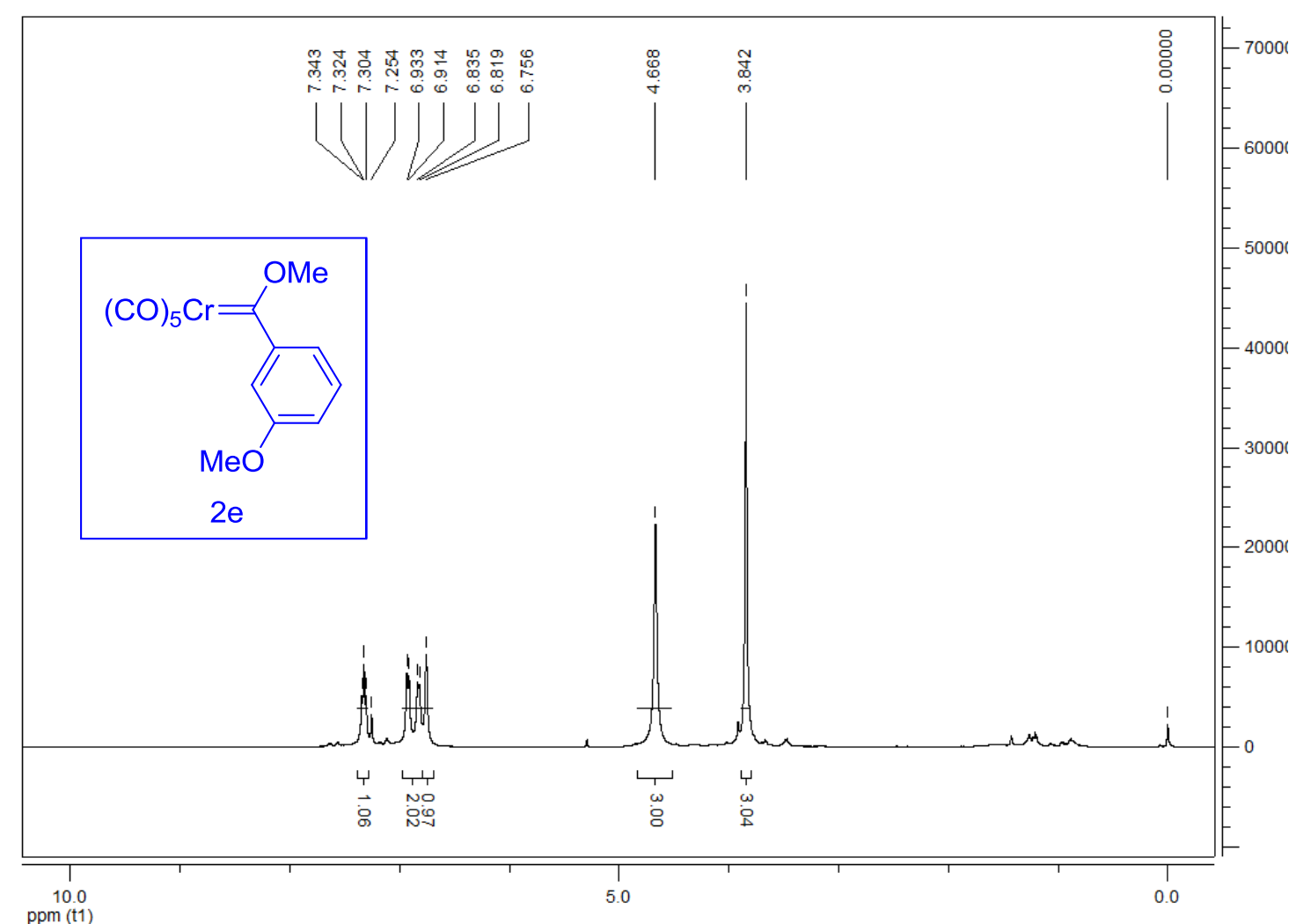

Figure S28. ${ }^{13} \mathrm{C}$ NMR of pentacarbonyl[(methoxy)(3-methoxyphenyl)carbene] chromium(0) (2e)

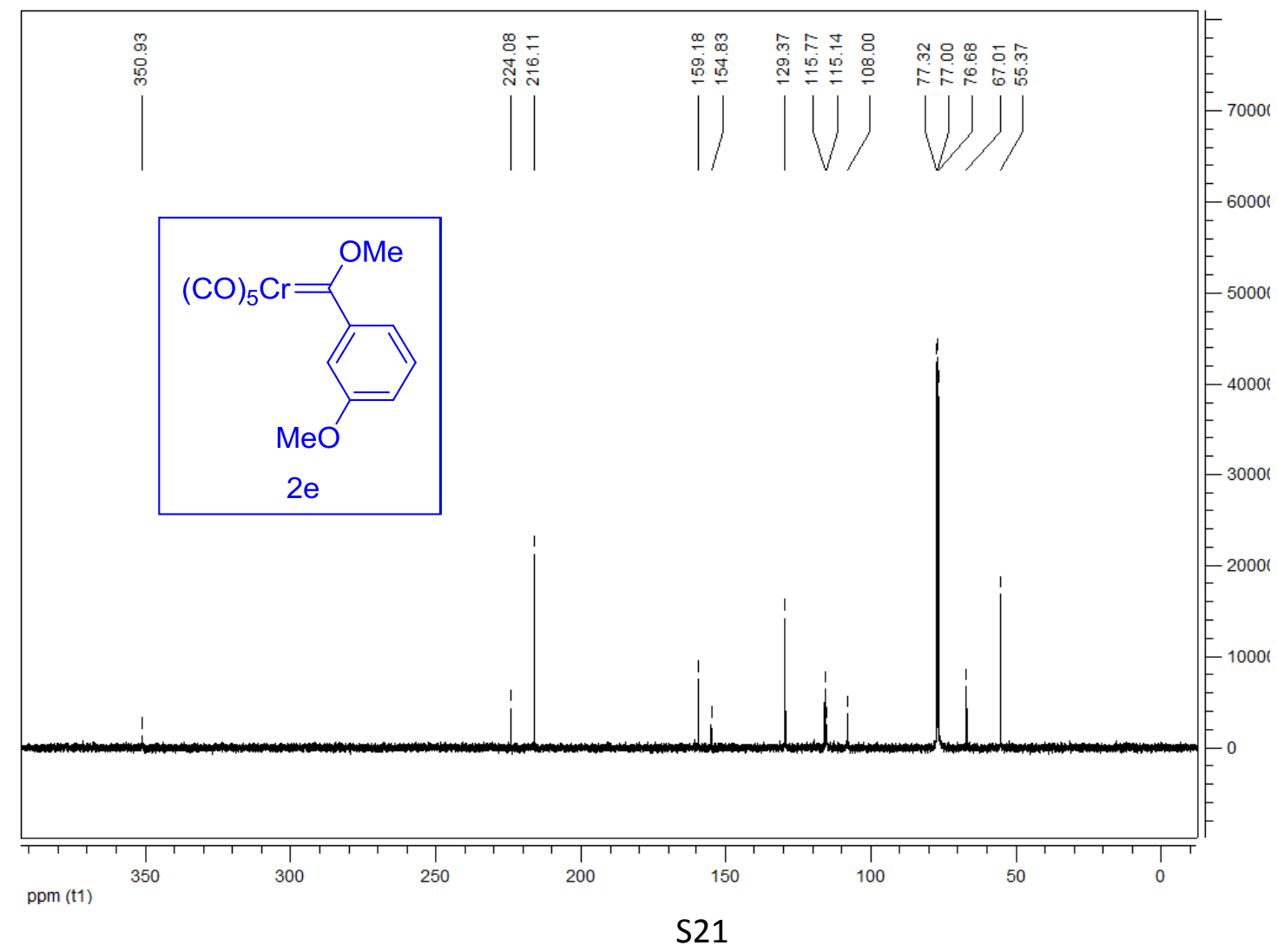


Figure S29. ${ }^{1}$ H NMR of pentacarbonyl[(methoxy)(4-methylphenyl)carbene]chromium(0) (2f)

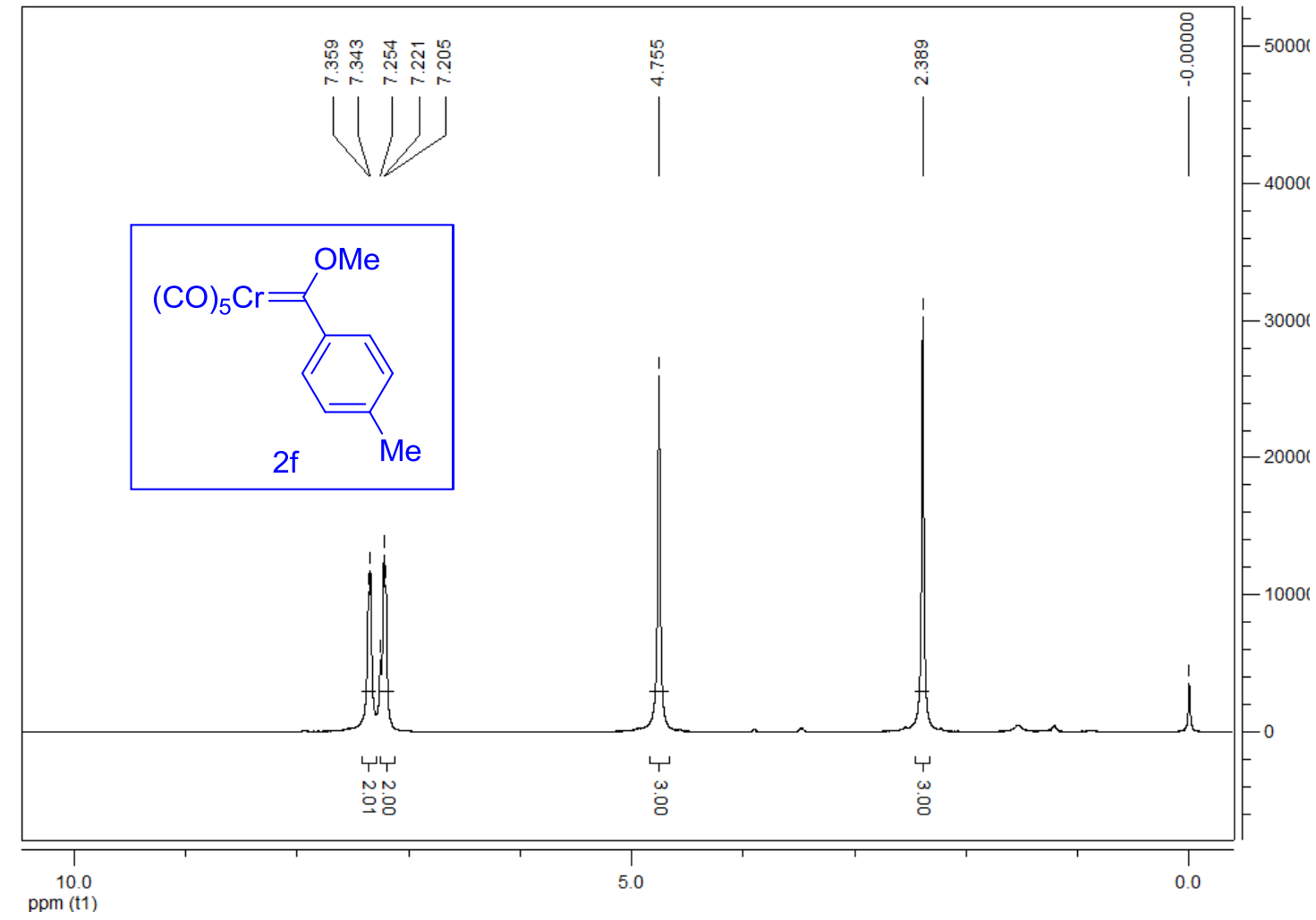

Figure S30. ${ }^{13} \mathrm{C}$ NMR of pentacarbonyl[(methoxy)(4-methylphenyl)carbene $]$ chromium(0) (2f)

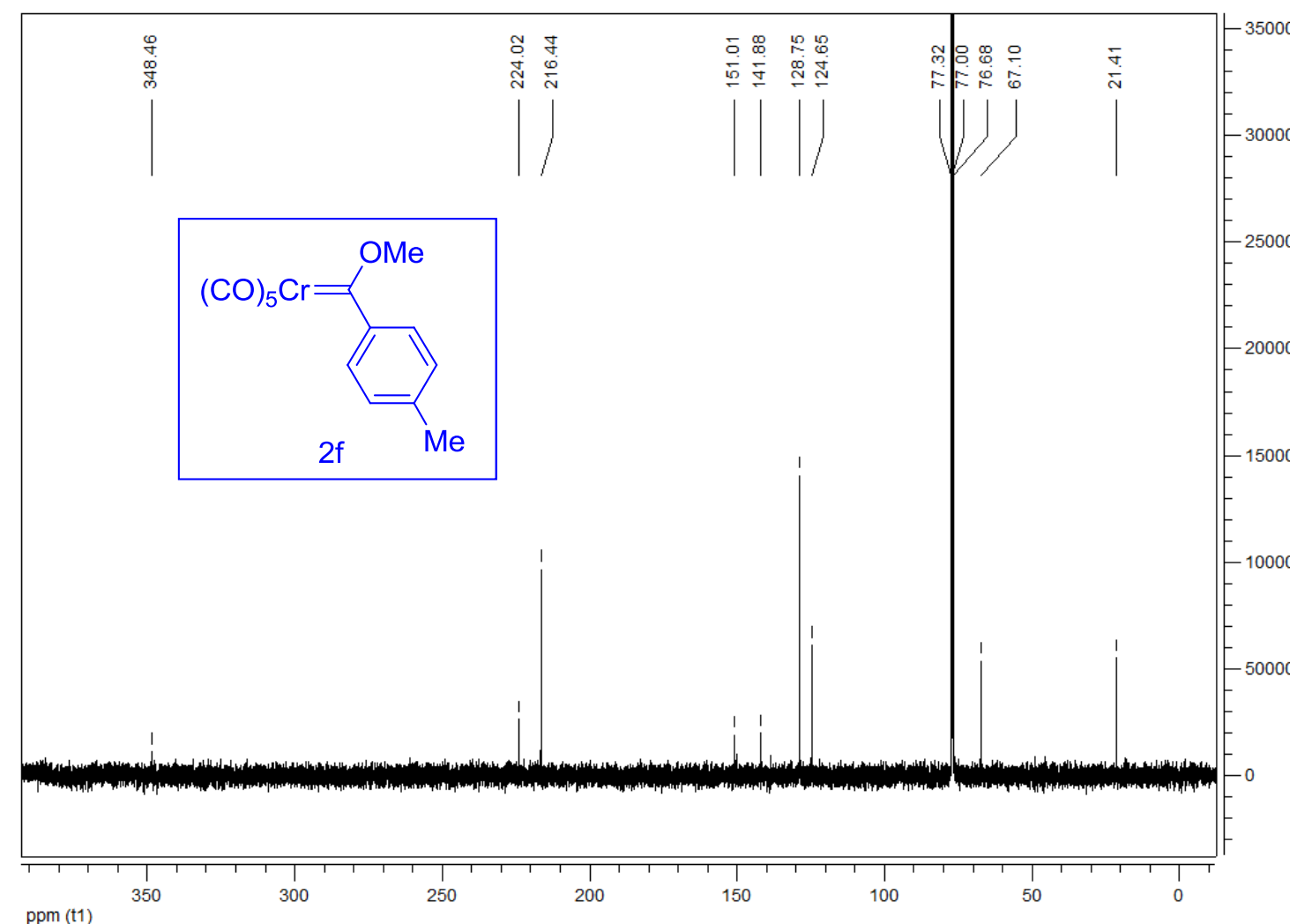


Figure S31. ${ }^{1} \mathrm{H}$ NMR of

pentacarbonyl[(methoxy)(4-methoxyphenyl)carbene]chromium(0) (2g)

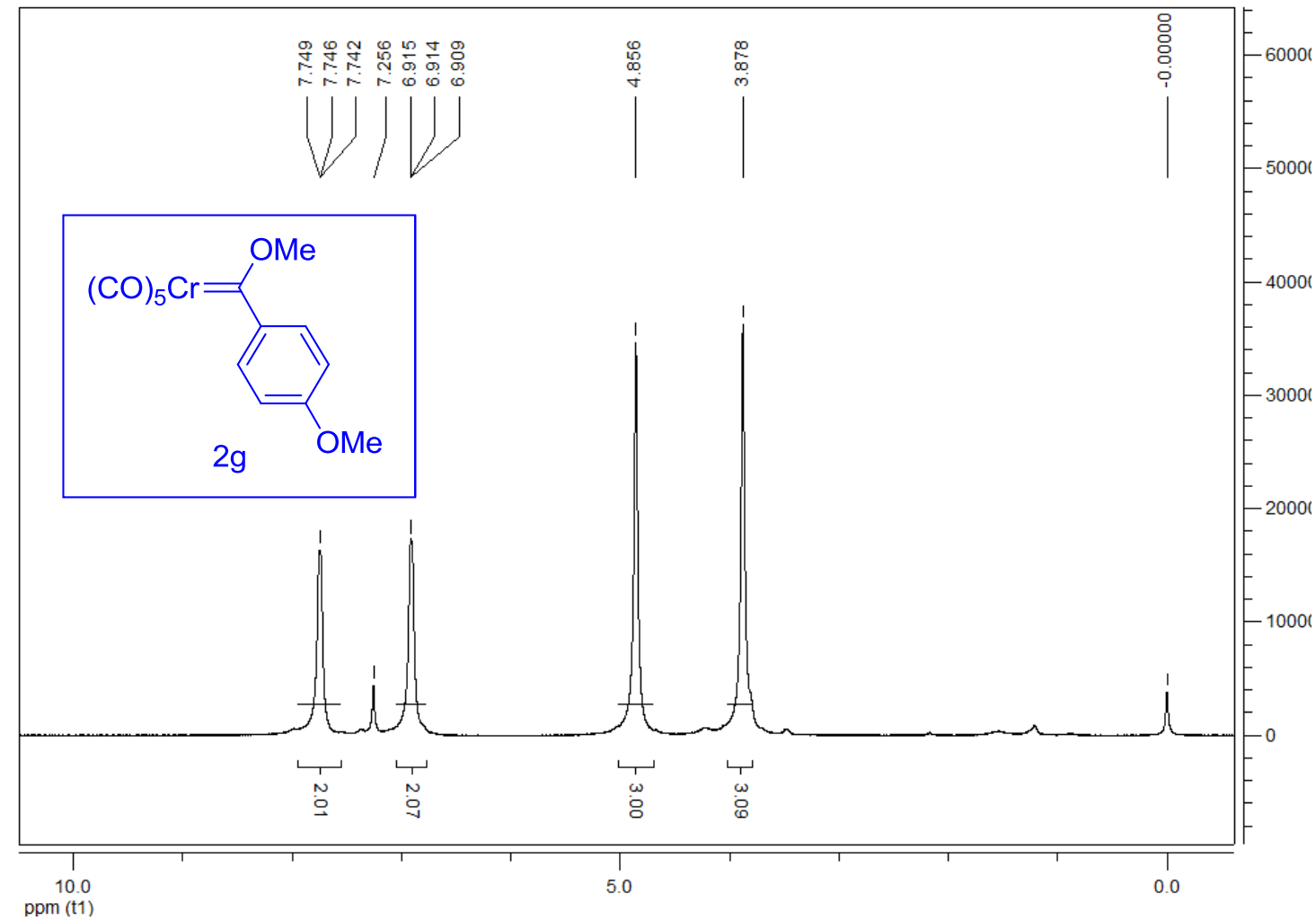

Figure S32. ${ }^{13} \mathrm{C}$ NMR of

pentacarbonyl[(methoxy)(4-methoxyphenyl)carbene $] \operatorname{chromium}(0)(2 \mathrm{~g})$

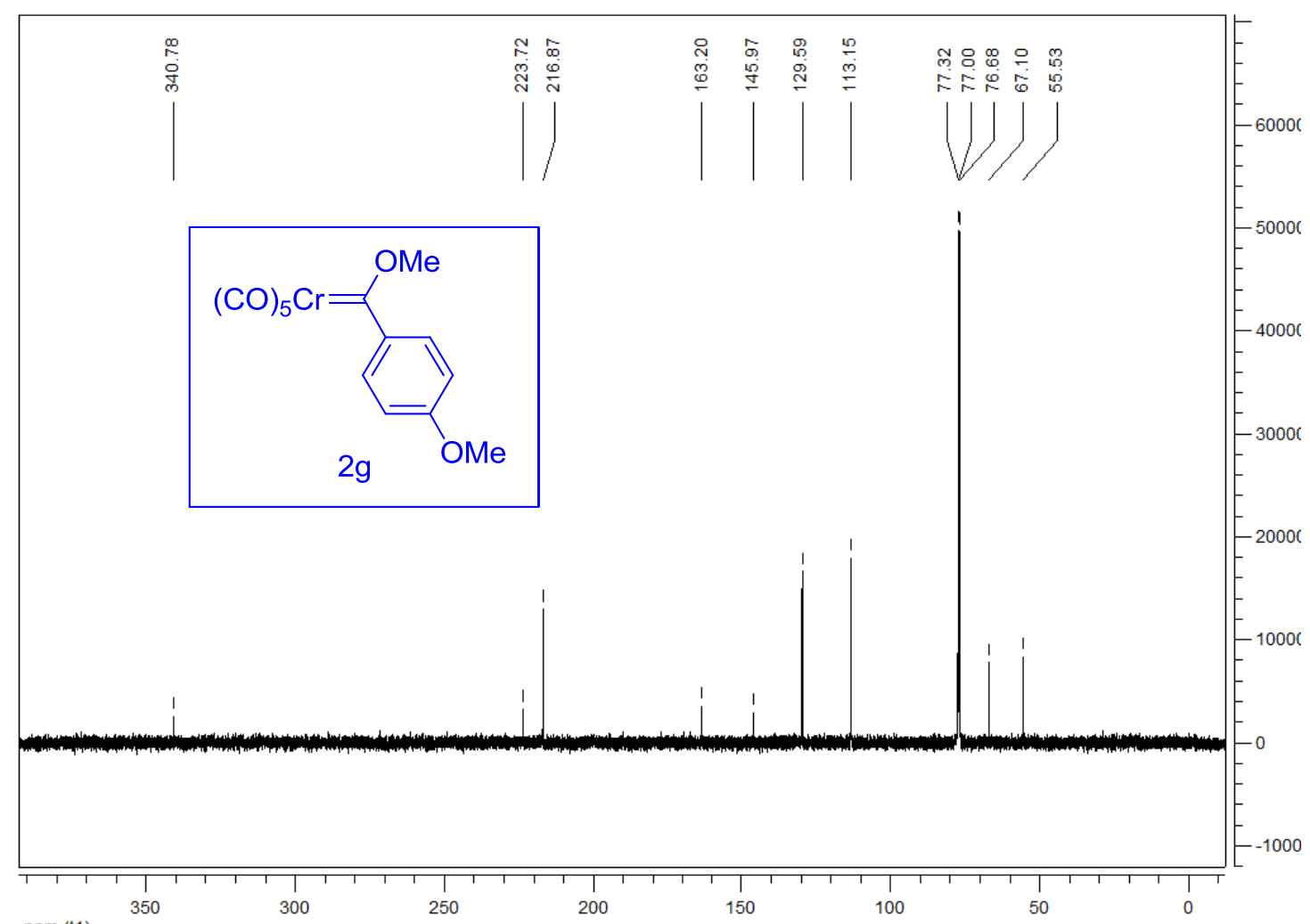

ppm (t1) 
Figure S33. ${ }^{1}$ H NMR of pentacarbonyl[(4-biphenyl)(methoxy)carbene $]$ chromium(0) (2h)

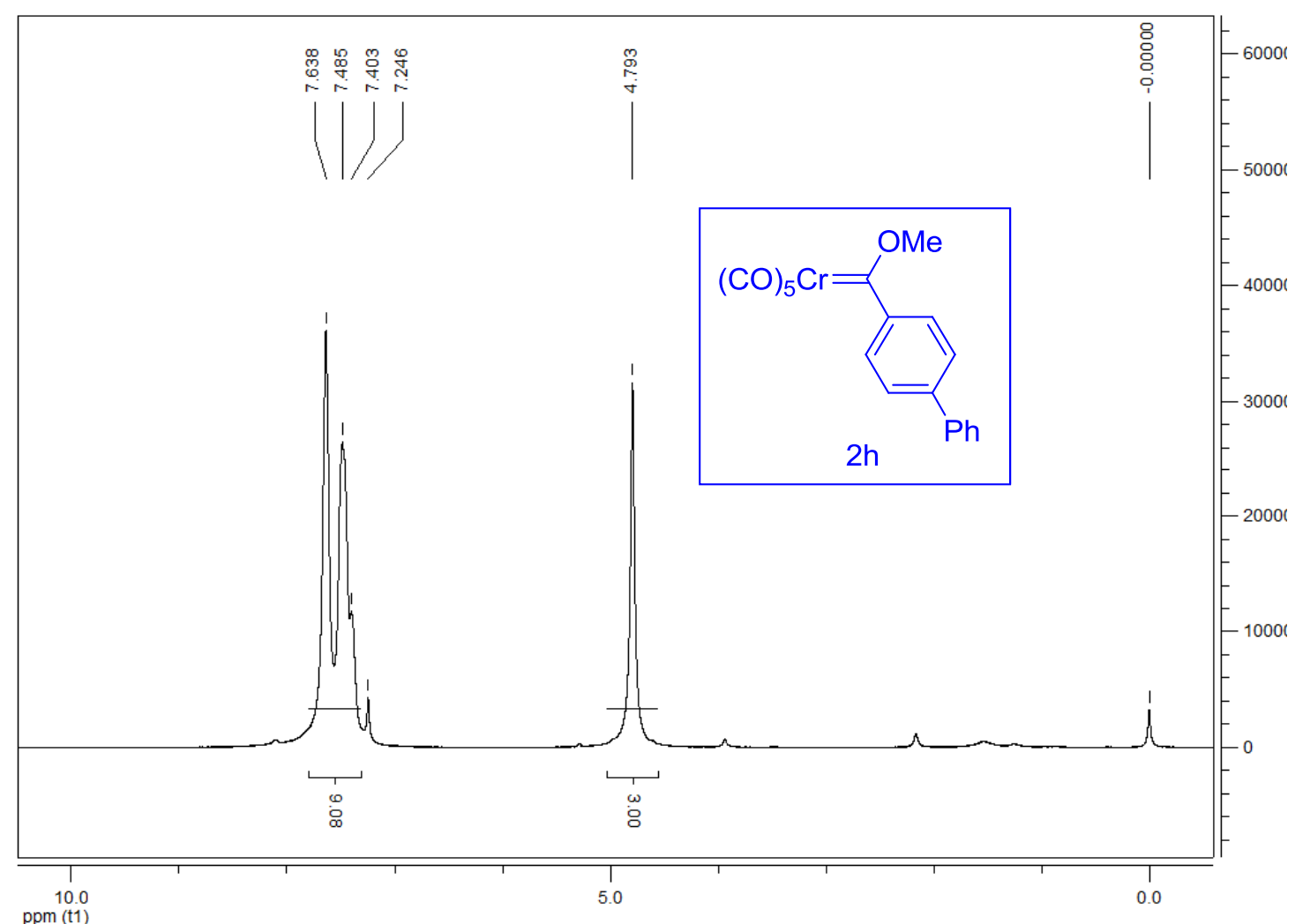

Figure S34. ${ }^{13} \mathrm{C}$ NMR of pentacarbonyl[(4-biphenyl)(methoxy)carbene]chromium(0) (2h)

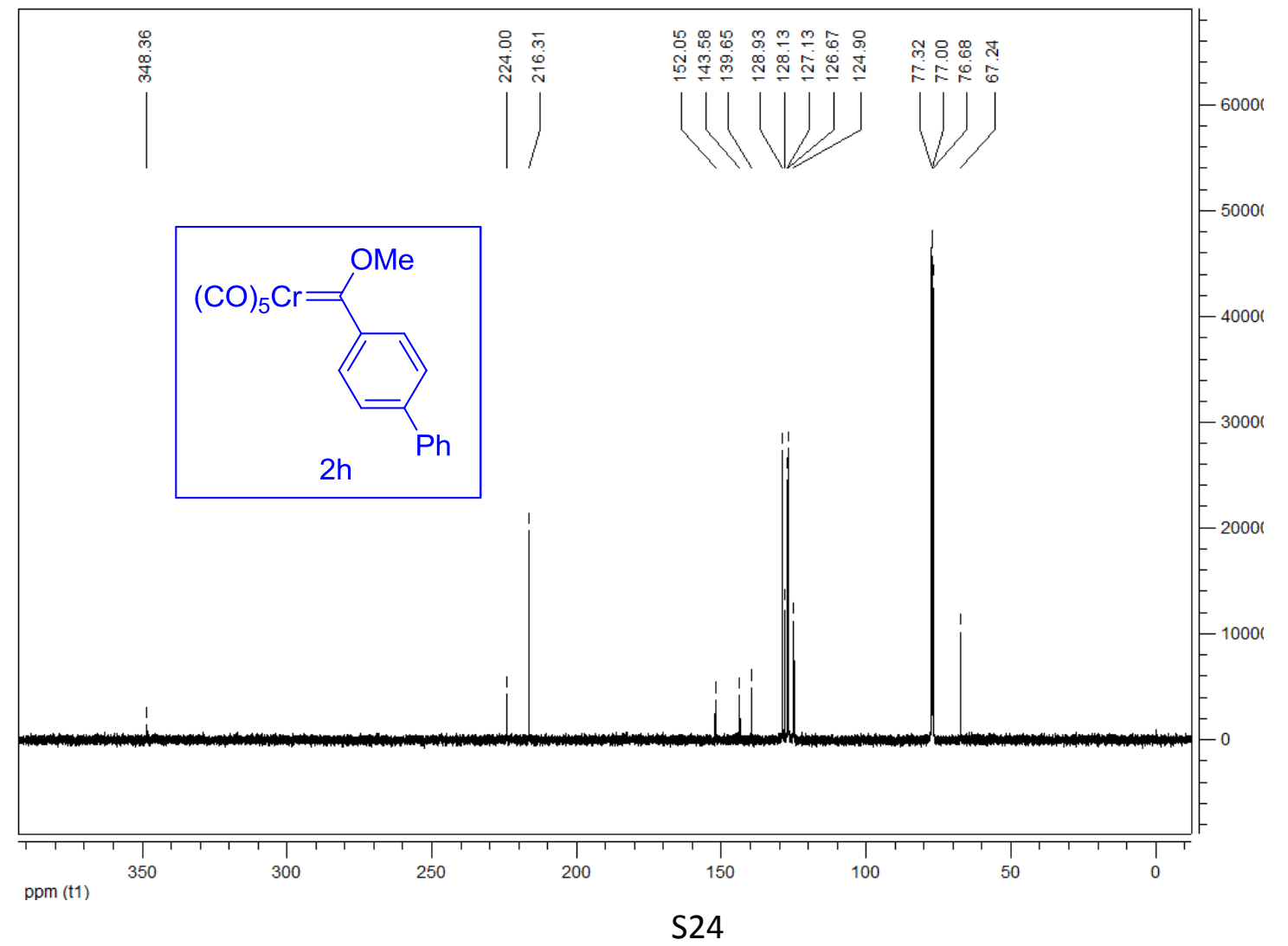


Figure S35. ${ }^{1} \mathrm{H}$ NMR of pentacarbonyl[(4-fluorophenyl)(methoxy)carbene]chromium(0) (2i)

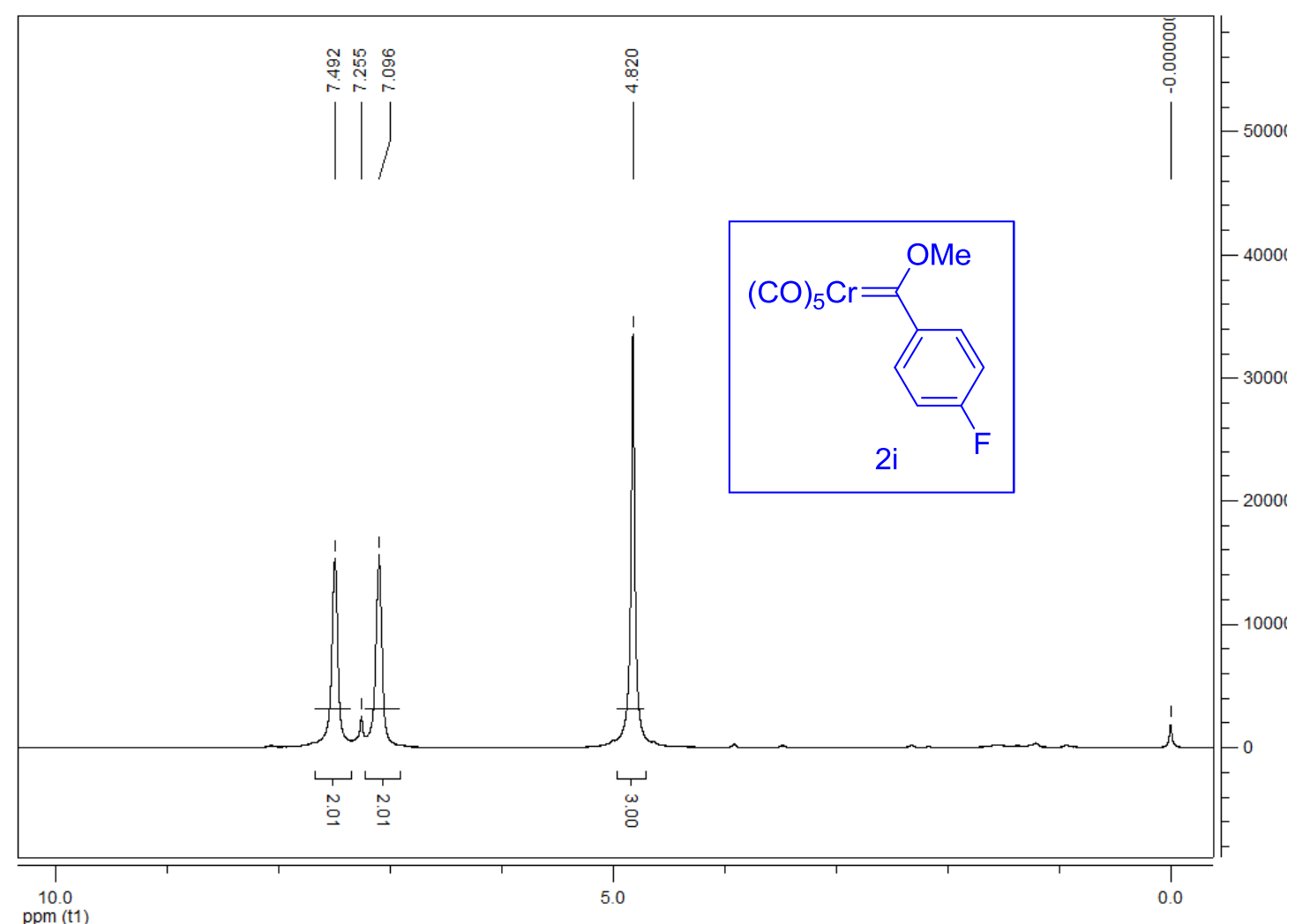

Figure S36. ${ }^{13} \mathrm{C}$ NMR of pentacarbonyl[(4-fluorophenyl)(methoxy)carbene $]$ chromium(0) (2i)

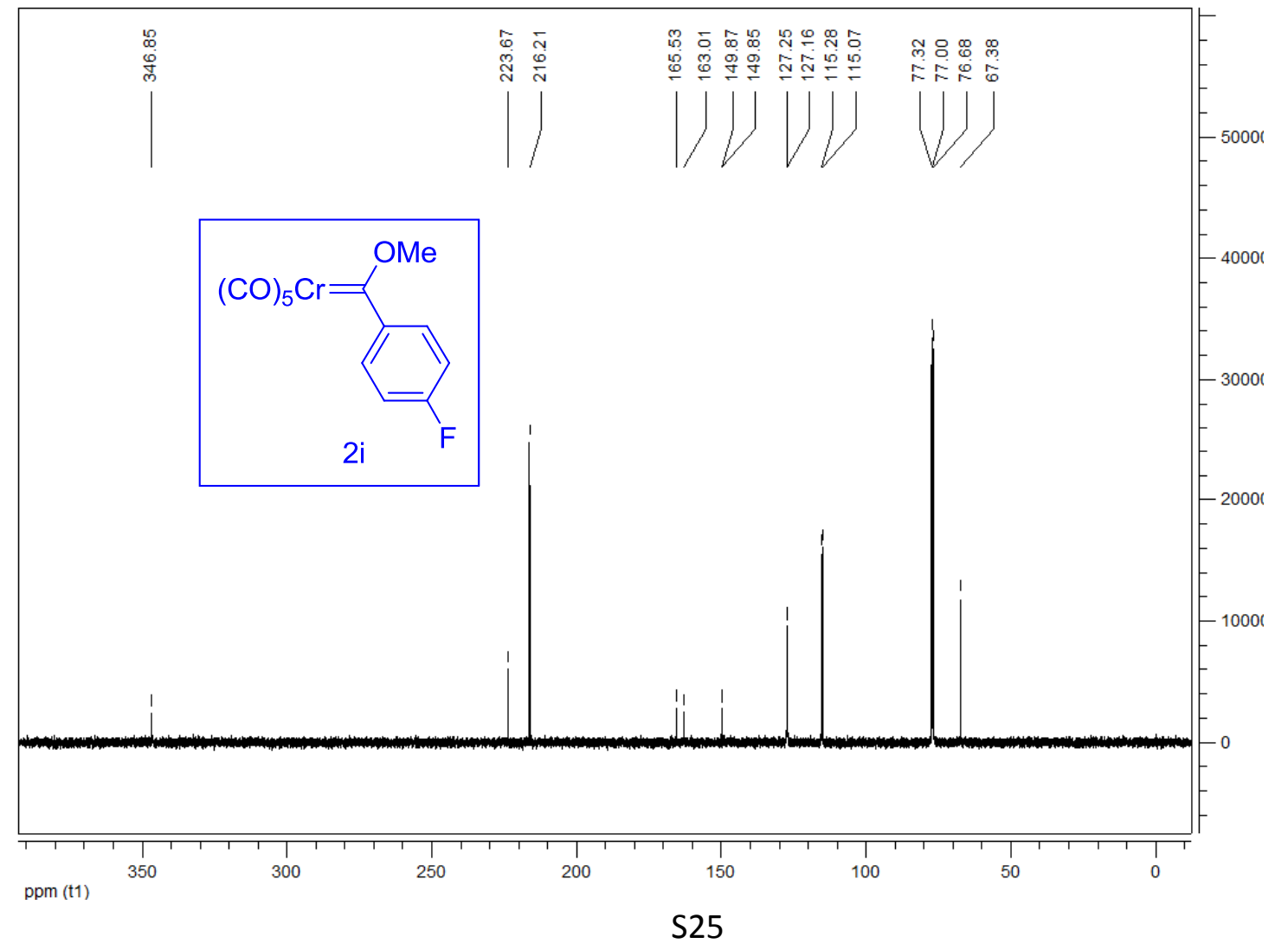


Figure S37. ${ }^{1} \mathrm{H}$ NMR of pentacarbonyl[(4-chlorophenyl)(methoxy)carbene]chromium(0) (2j)

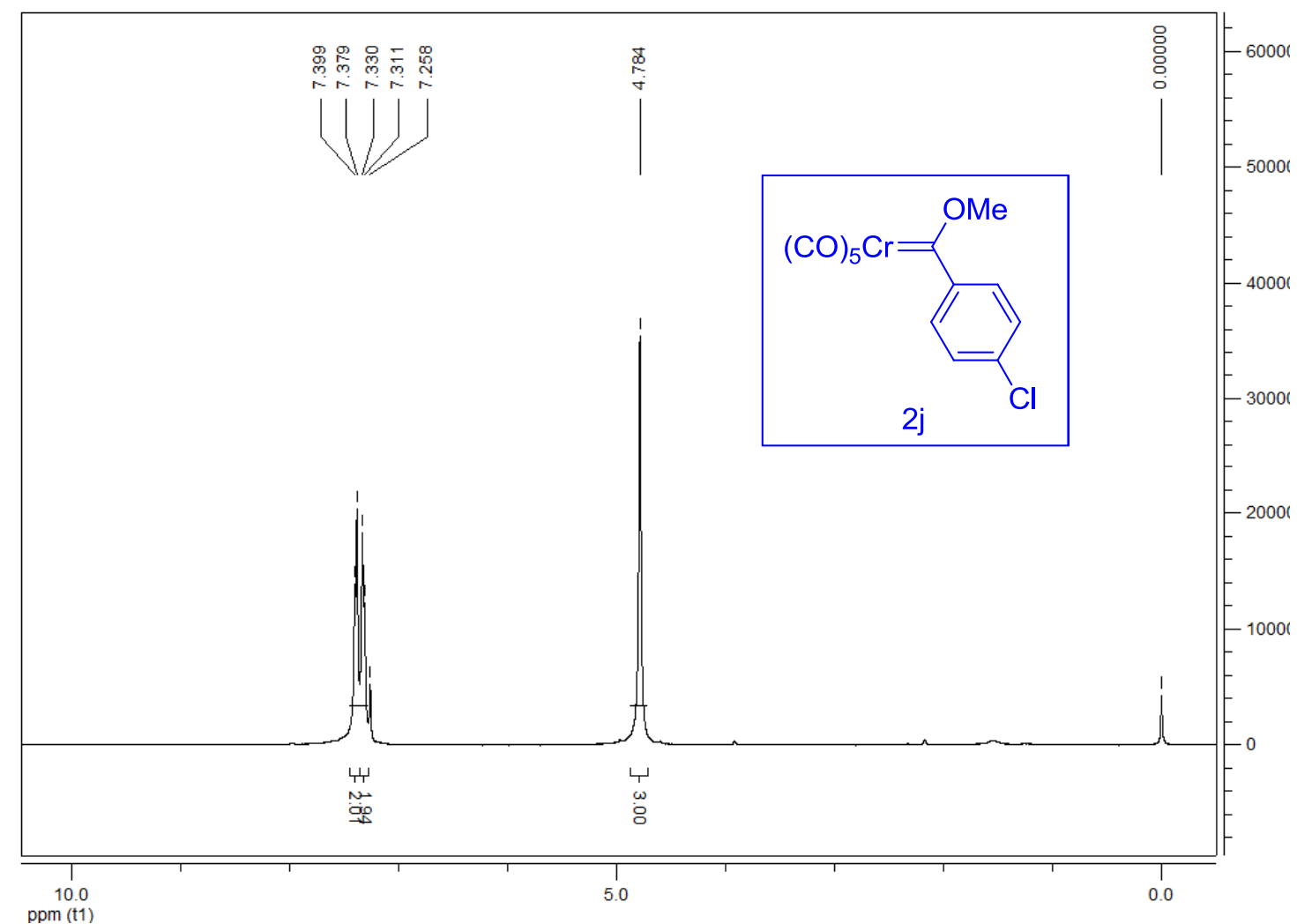

Figure S38. ${ }^{13} \mathrm{C}$ NMR of pentacarbonyl[(4-chlorophenyl)(methoxy)carbene $] \operatorname{chromium}(0)$ (2j)

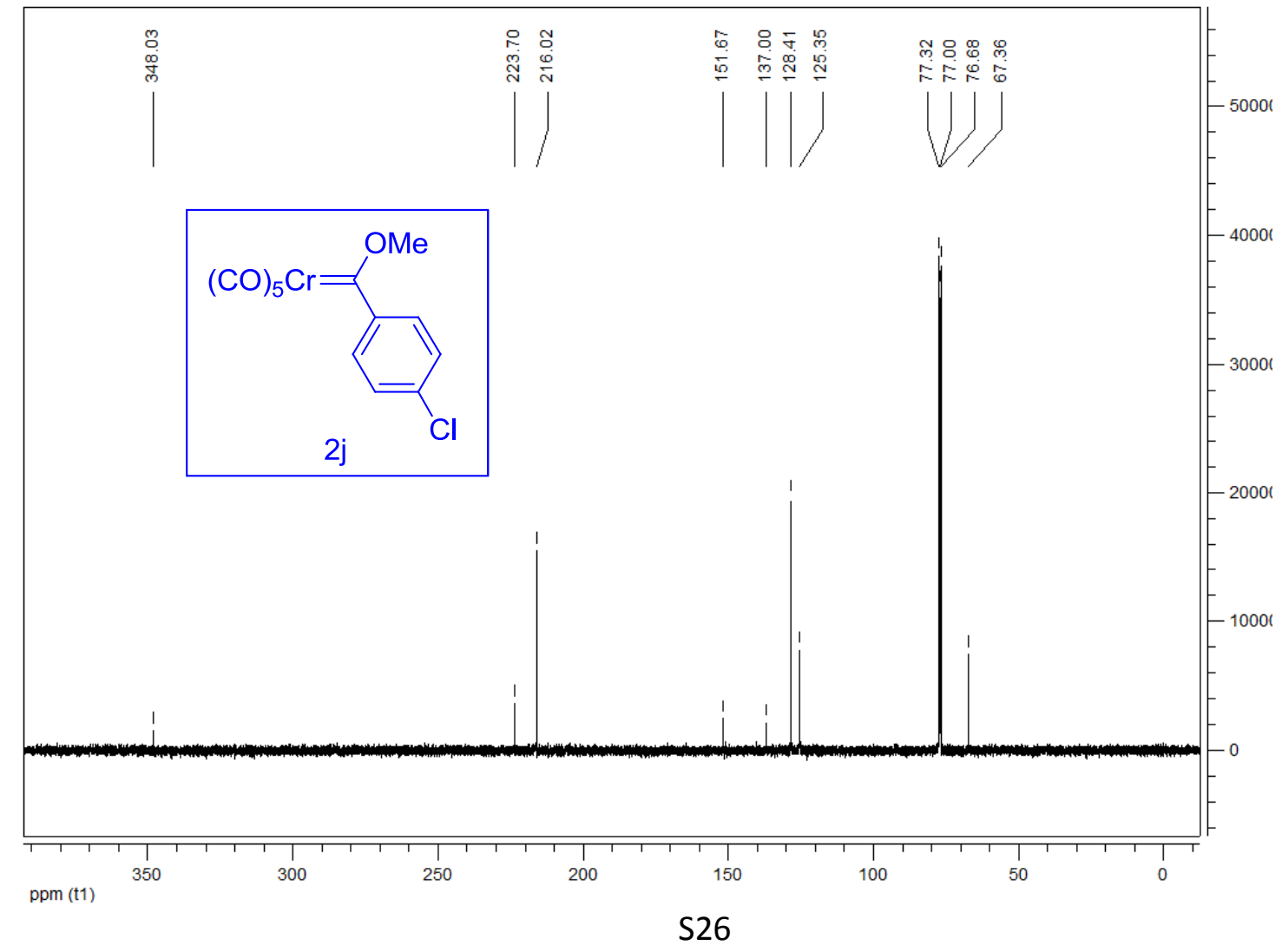


Figure S39. ${ }^{1} \mathrm{H}$ NMR of

pentacarbonyl[(methoxy)(4-trifluoromethylphenyl)carbene]chromium(0) (2k)

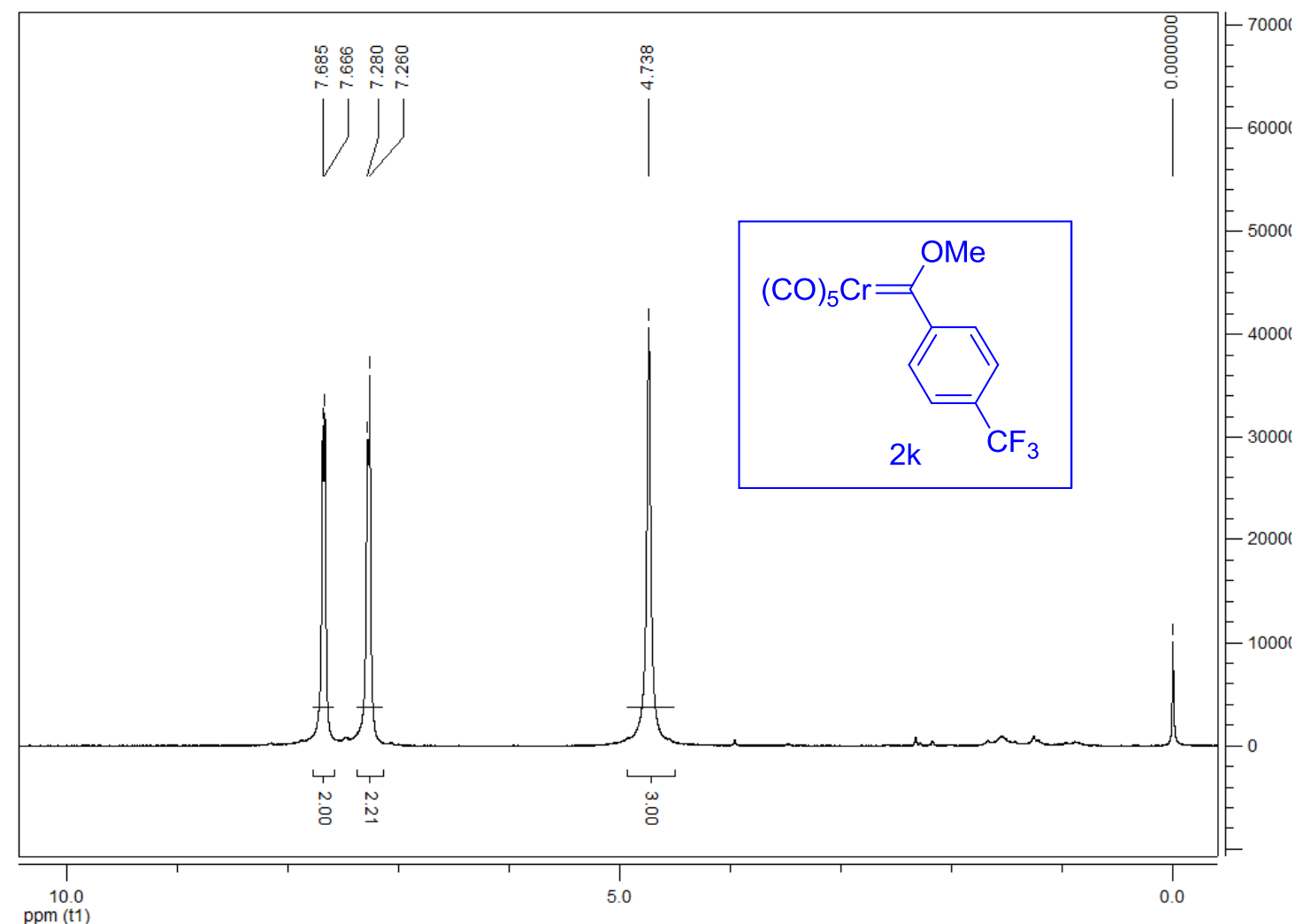

Figure S40. ${ }^{13} \mathrm{C}$ NMR of

pentacarbonyl[(methoxy)(4-trifluoromethylphenyl)carbene]chromium(0) (2k)

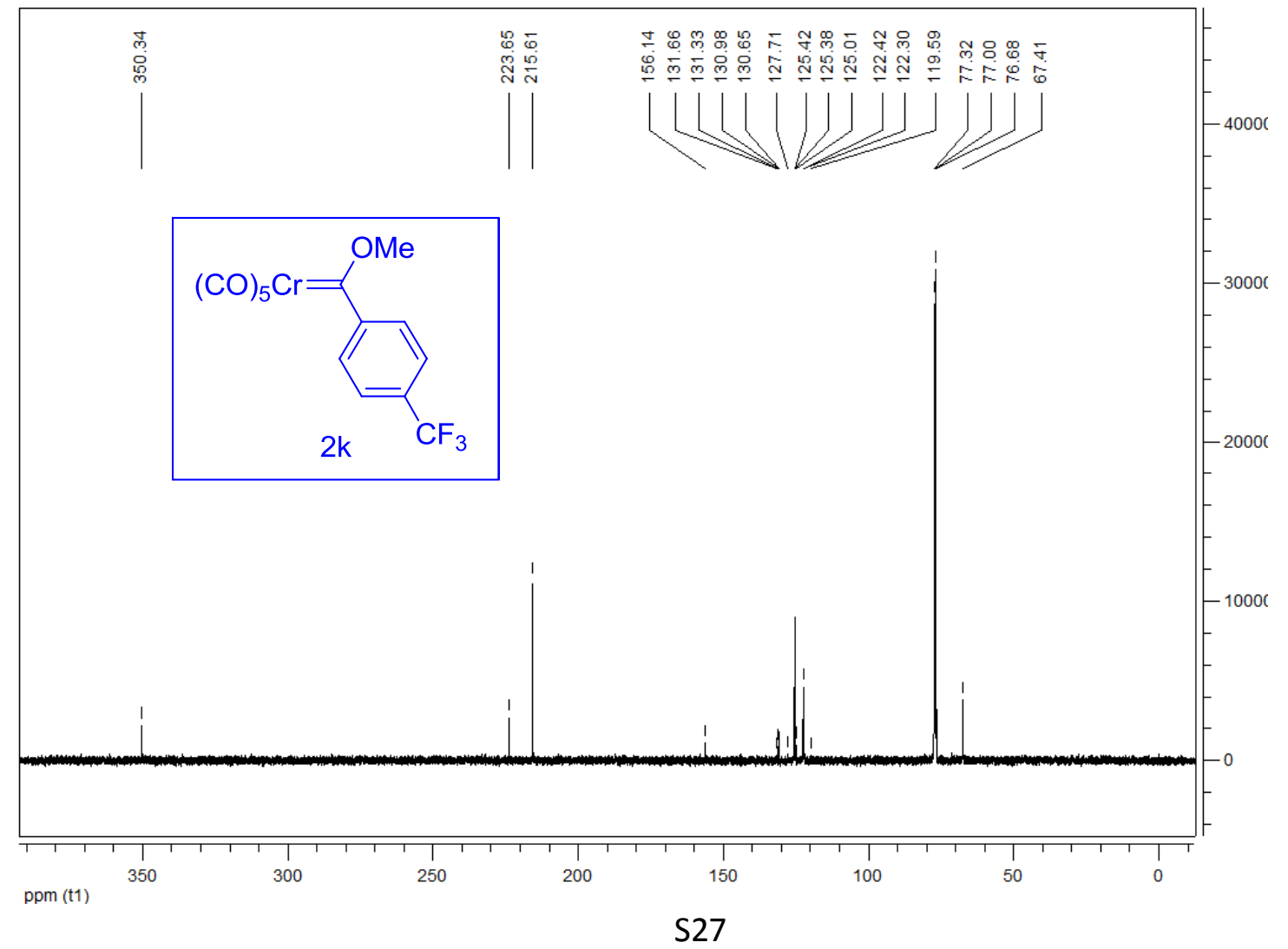


Figure S41. ${ }^{1}$ H NMR of pentacarbonyl[(3-furyl)(methoxy)carbene]chromium(0) (2l)

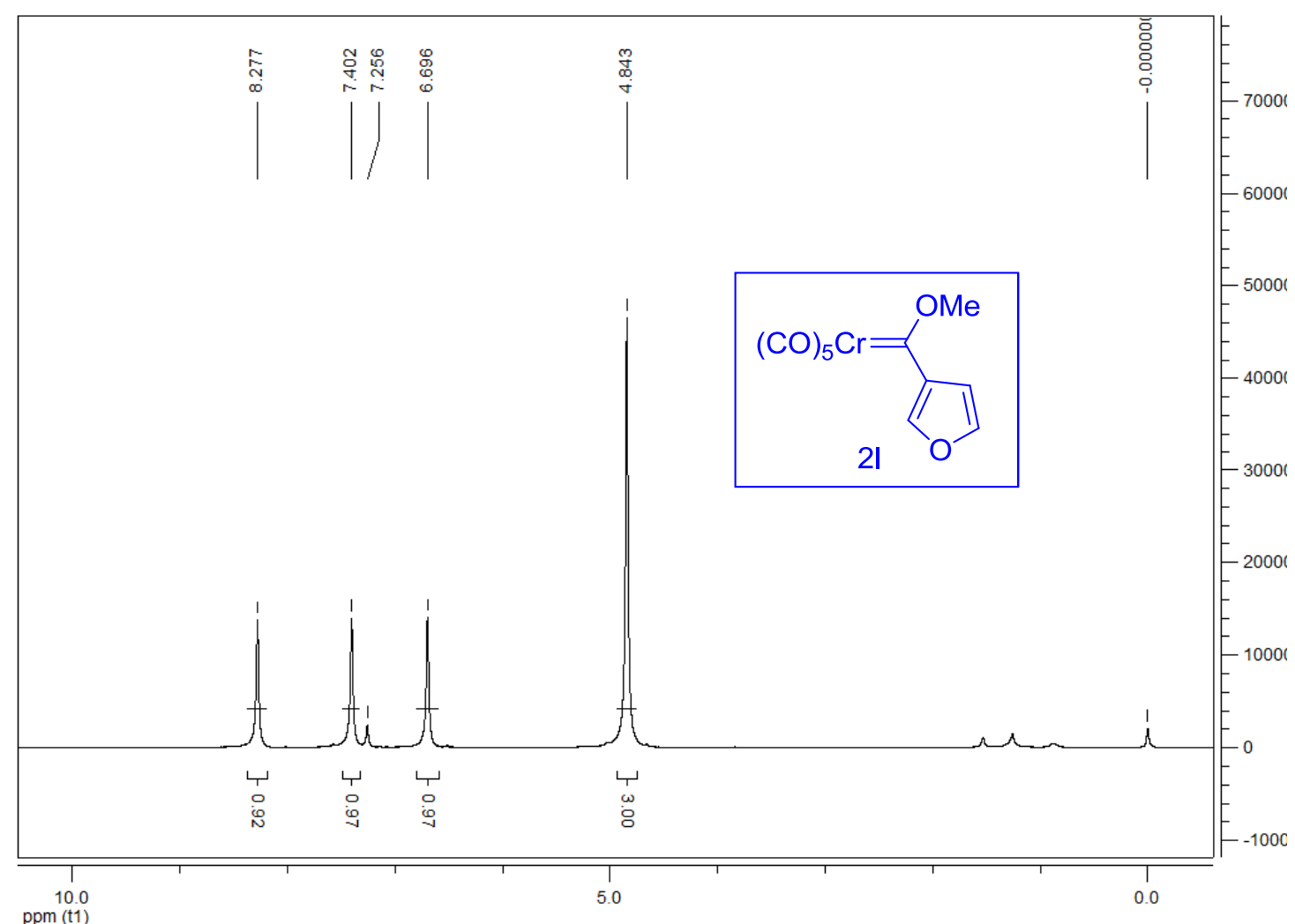

Figure S42. ${ }^{13} \mathrm{C}$ NMR of pentacarbonyl[(3-furyl)(methoxy)carbene $]$ chromium(0) (2I)

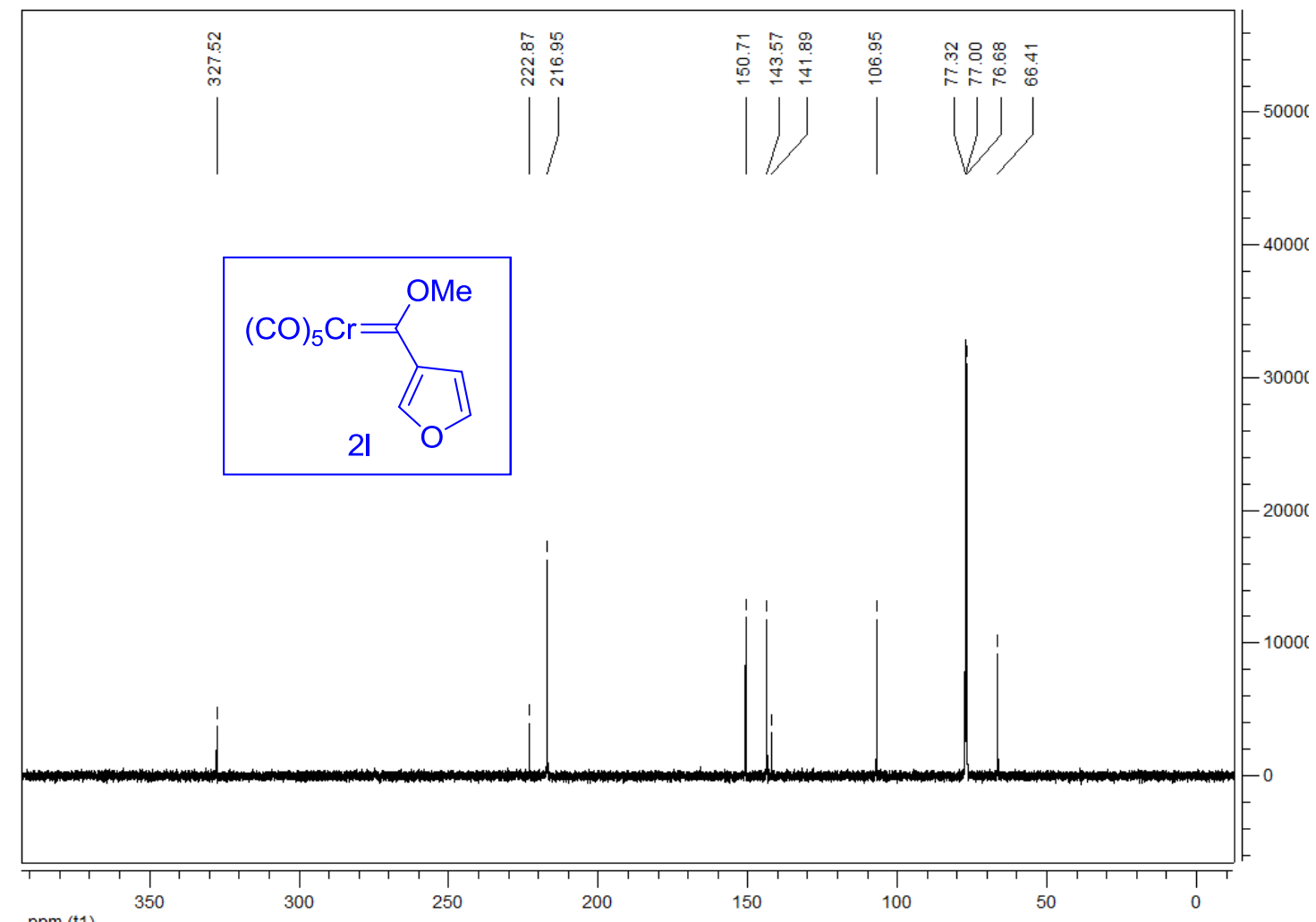


Figure S43. ${ }^{1} \mathrm{H}$ NMR of pentacarbonyl[(2-thiofuryl)(methoxy)carbene]chromium(0) (2m)

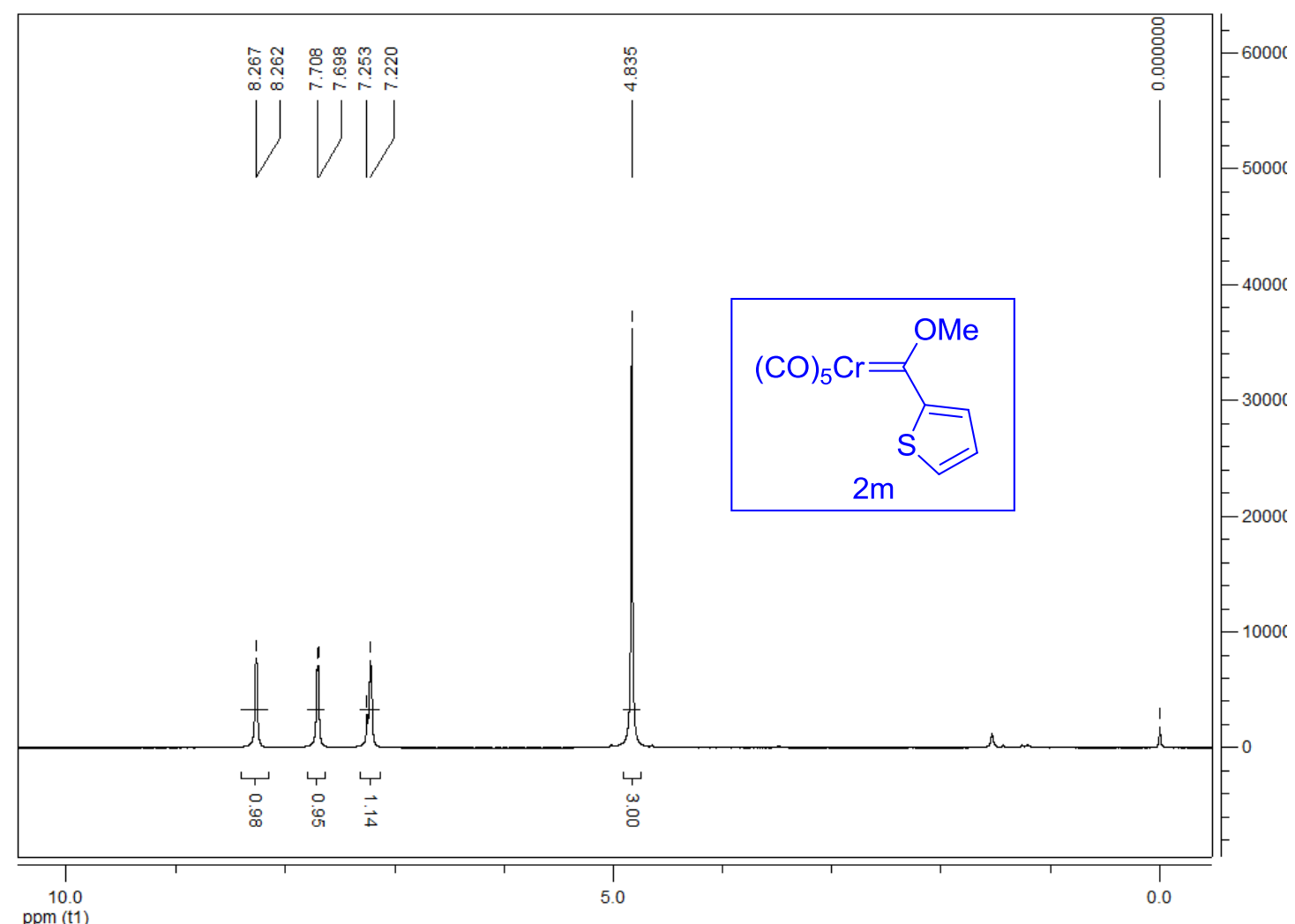

Figure S44. ${ }^{13} \mathrm{C}$ NMR of pentacarbonyl[(2-thiofuryl)(methoxy)carbene]chromium(0) (2m)

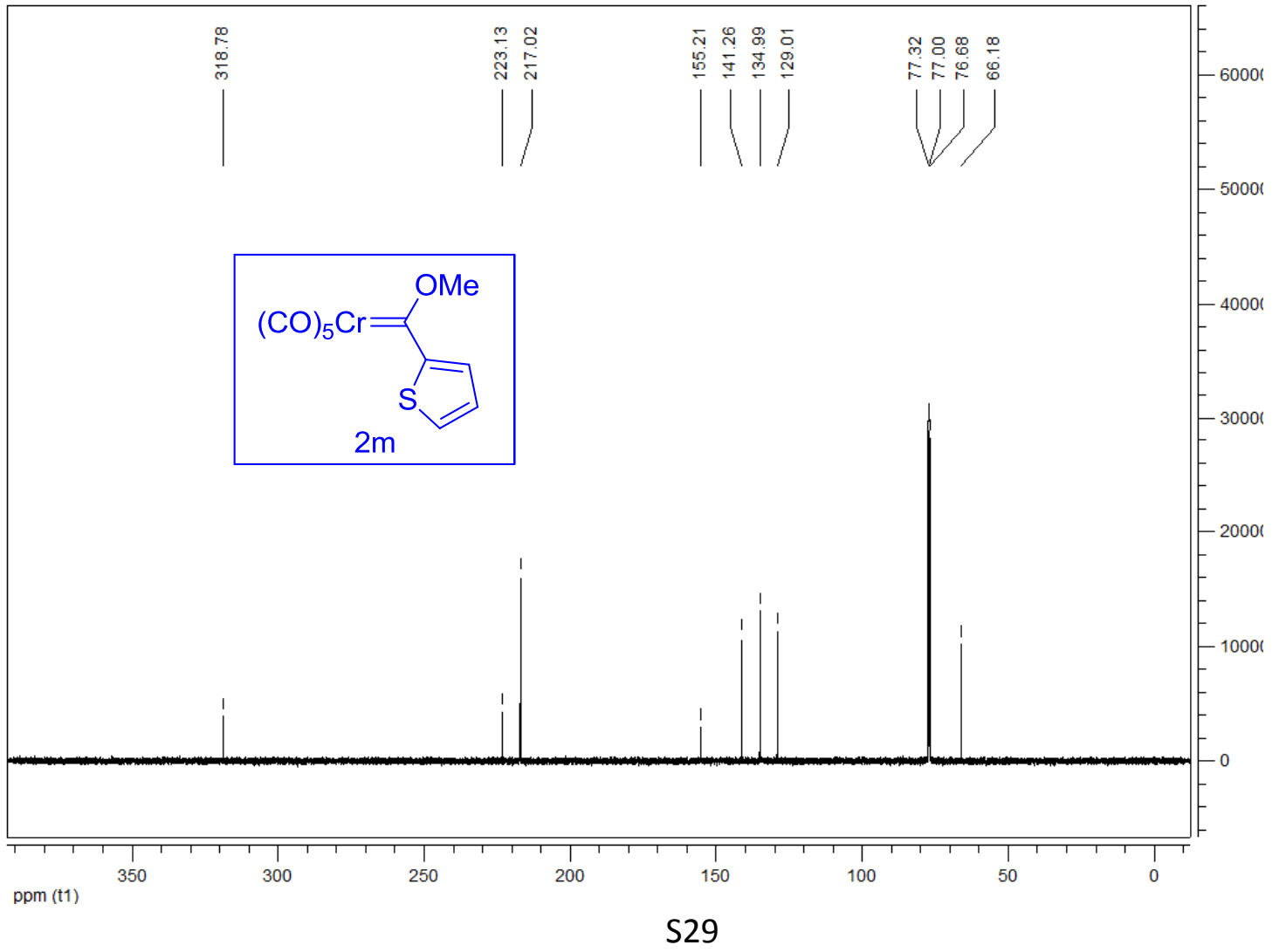


Figure S45. ${ }^{1} \mathrm{H}$ NMR of pentacarbonyl[methoxy(2-naphthalenyl)carbene]chromium(0) (2n)

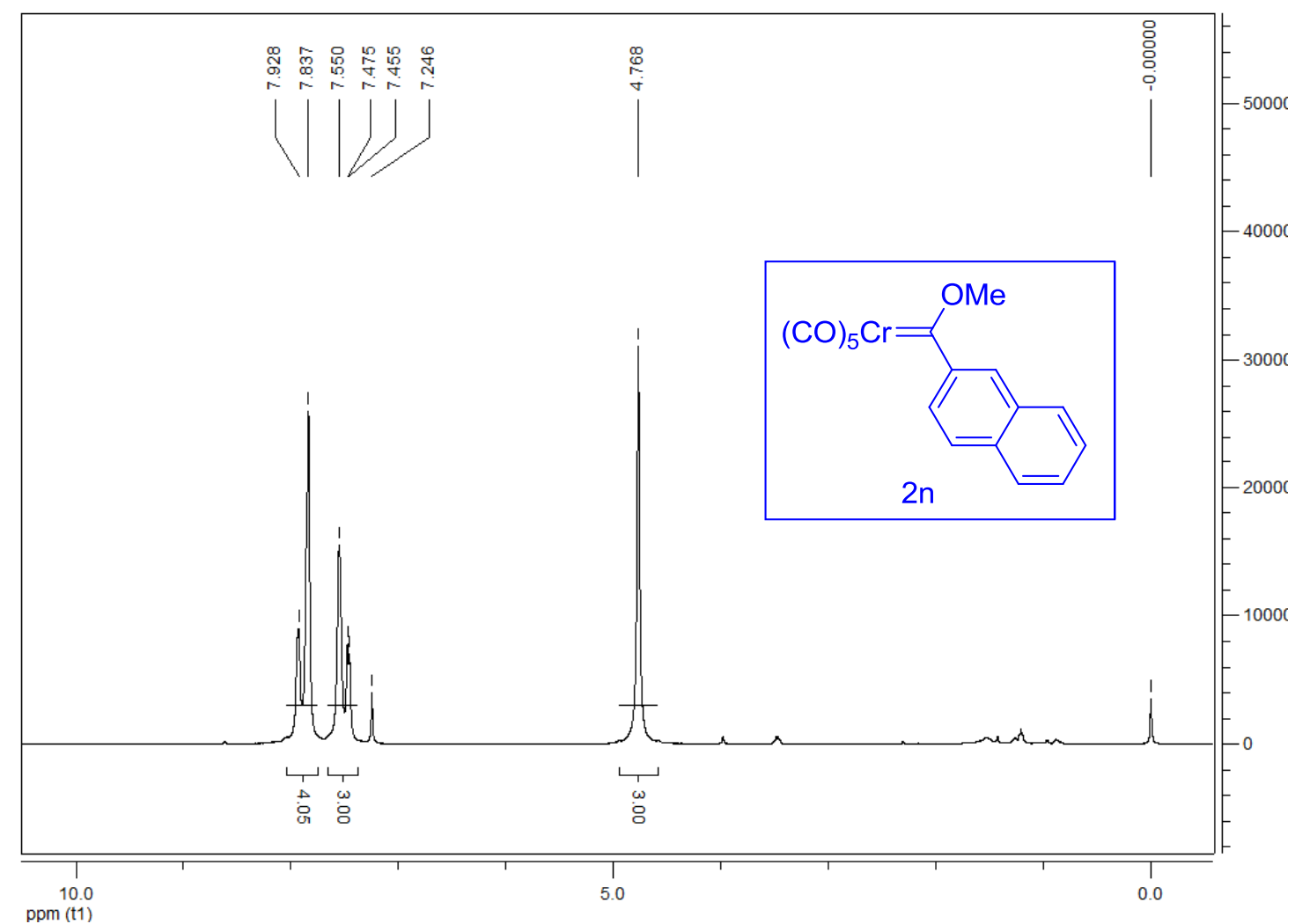

Figure S46. ${ }^{13} \mathrm{C}$ NMR of pentacarbonyl[methoxy(2-naphthalenyl)carbene]chromium(0) (2n)

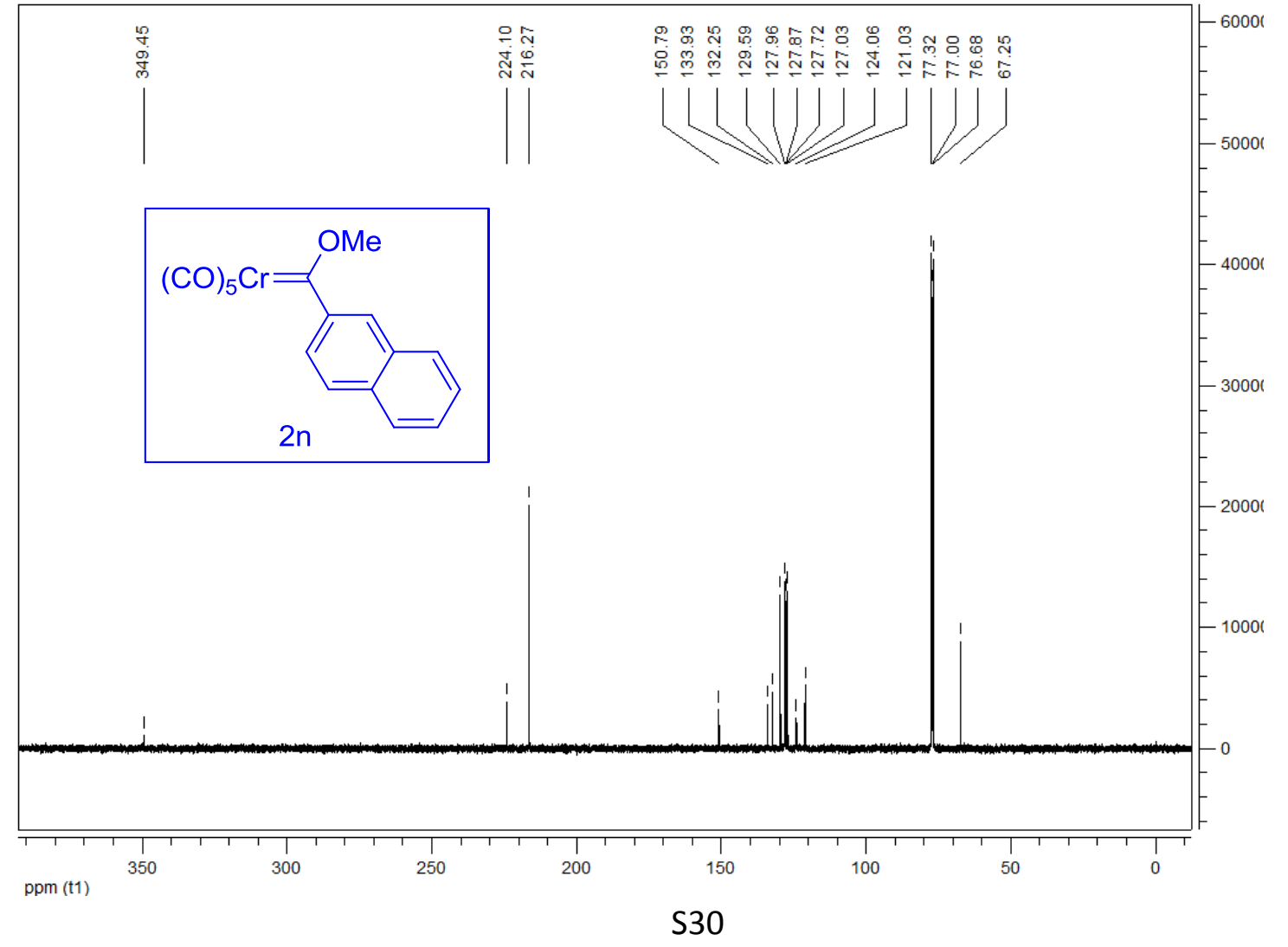


Figure S47. ${ }^{1} \mathrm{H}$ NMR of pentacarbonyl[(ethoxy)(phenyl)carbene $]$ chromium(0) (2o)

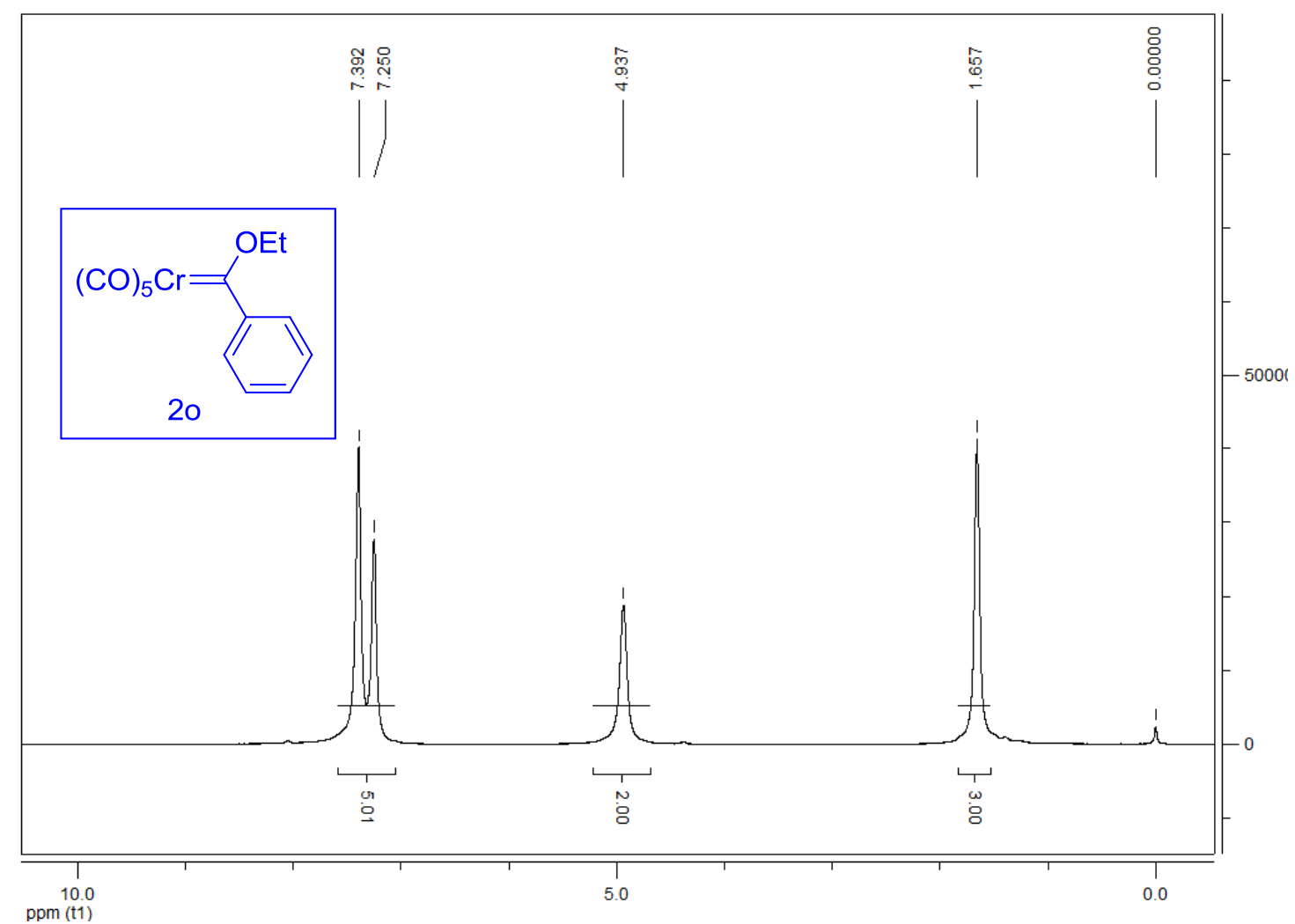

Figure S48. ${ }^{13} \mathrm{C}$ NMR of pentacarbonyl[(ethoxy)(phenyl)carbene $]$ chromium(0) (2o)

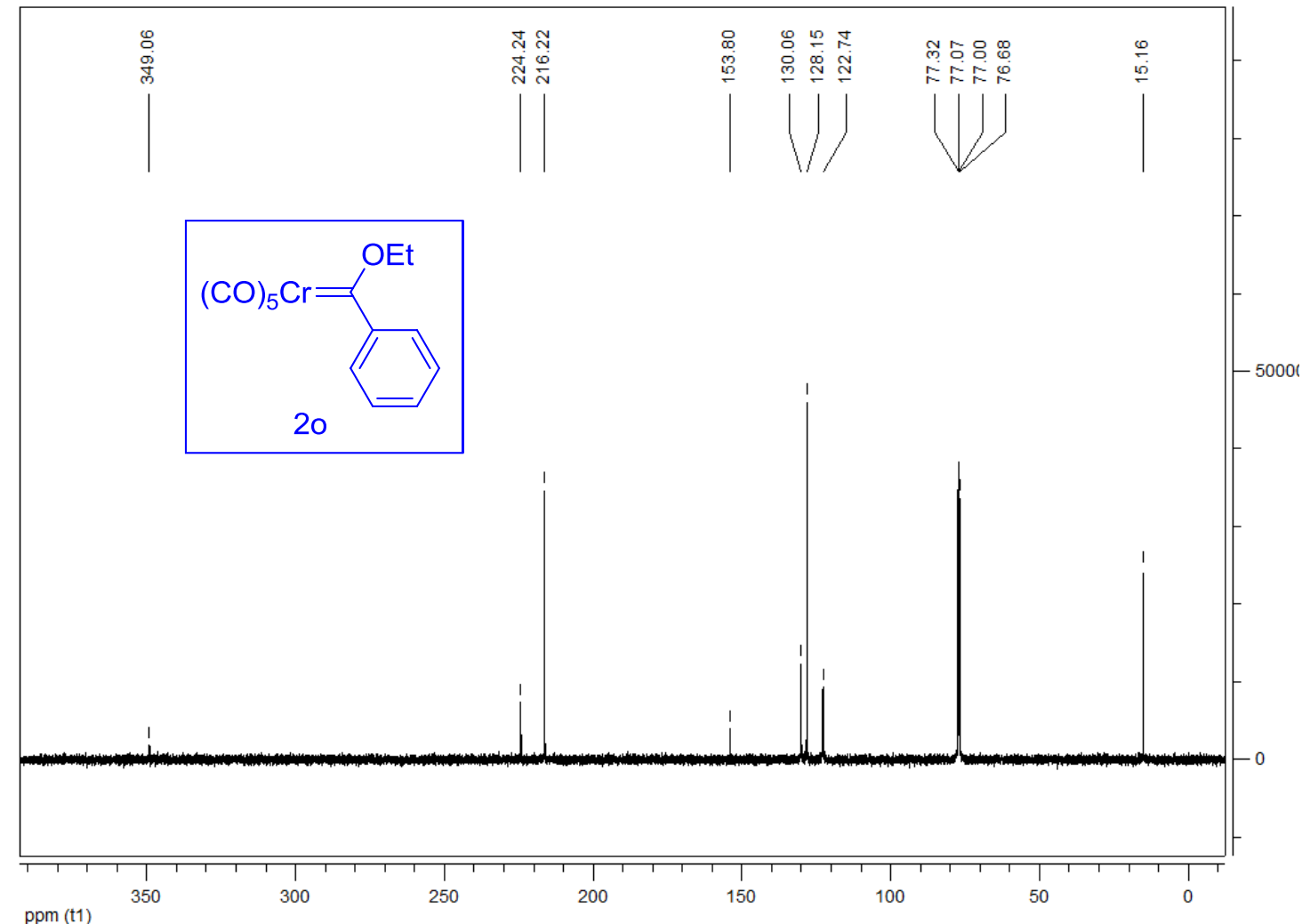


Figure S49. ${ }^{1} \mathrm{H}$ NMR of 2-(methoxy(phenyl)methyl)benzo[d]oxazole (3a)

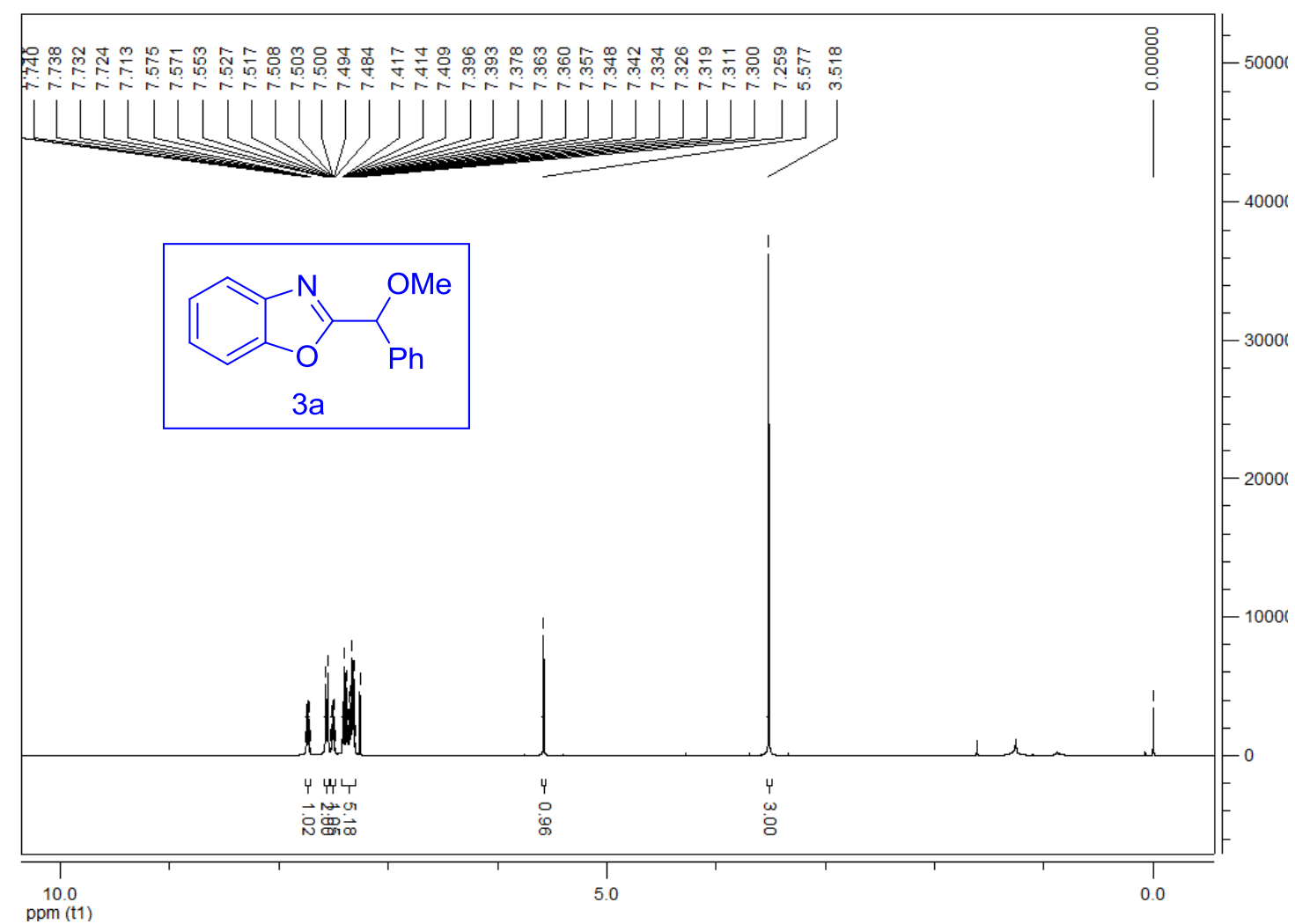

Figure S50. ${ }^{13} \mathrm{C}$ NMR of 2-(methoxy(phenyl)methyl)benzo[d]oxazole (3a)

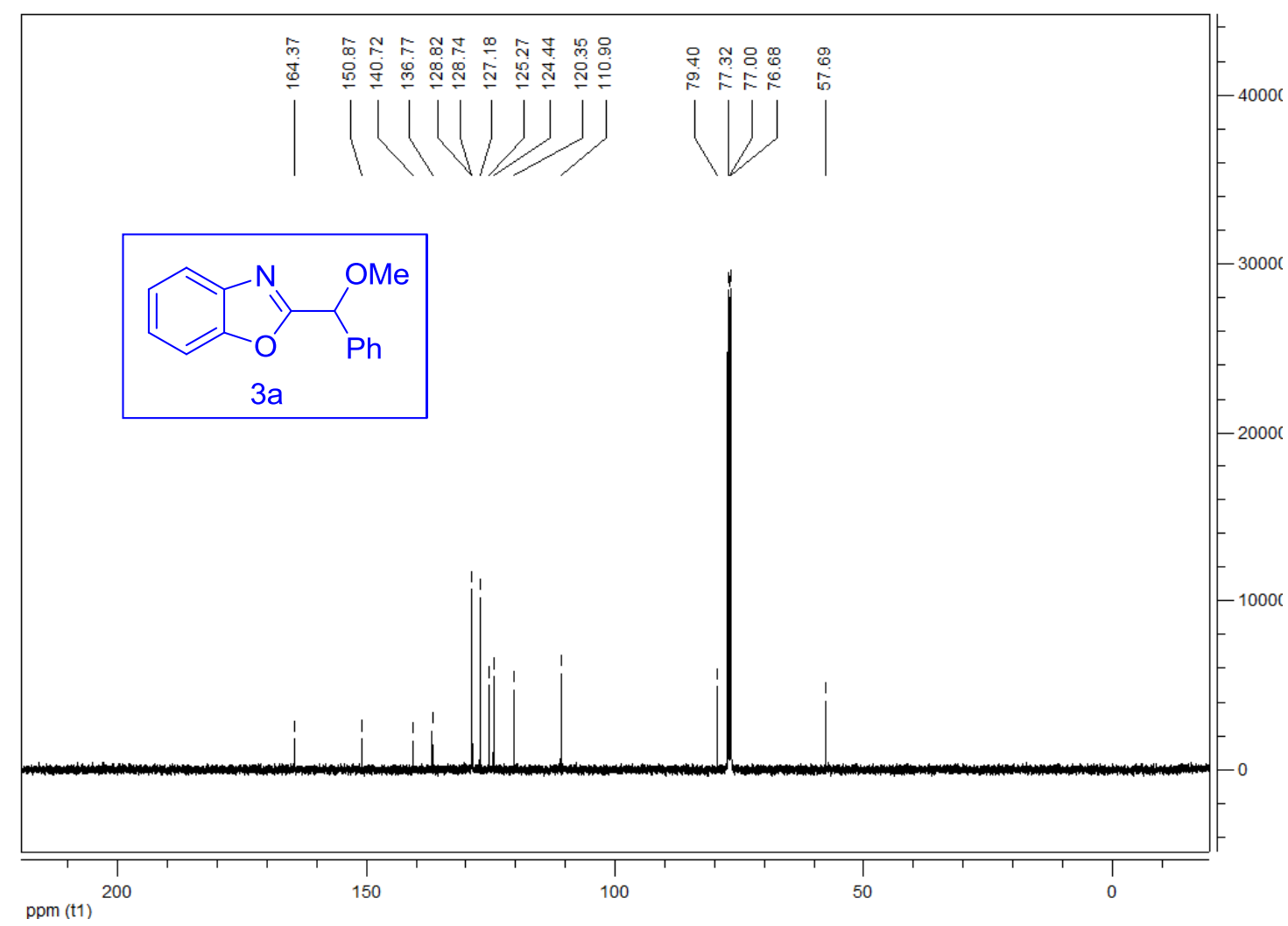


Figure S51. ${ }^{1} \mathrm{H}$ NMR of 2-(methoxy(phenyl)methyl)-4-methylbenzo[d]oxazole (3b)

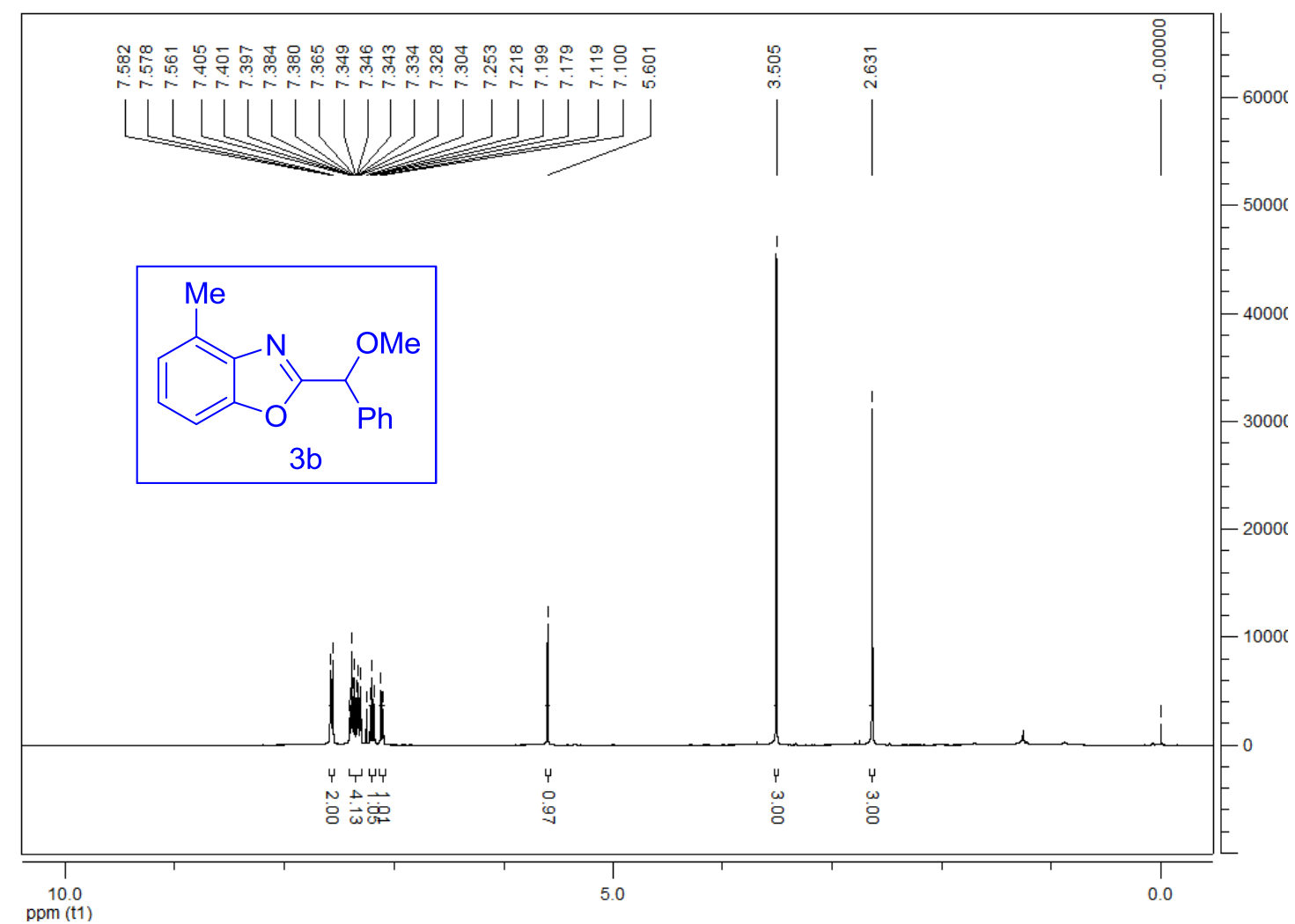

Figure S52. ${ }^{13} \mathrm{C}$ NMR of 2-(methoxy(phenyl)methyl)-4-methylbenzo[d]oxazole (3b)

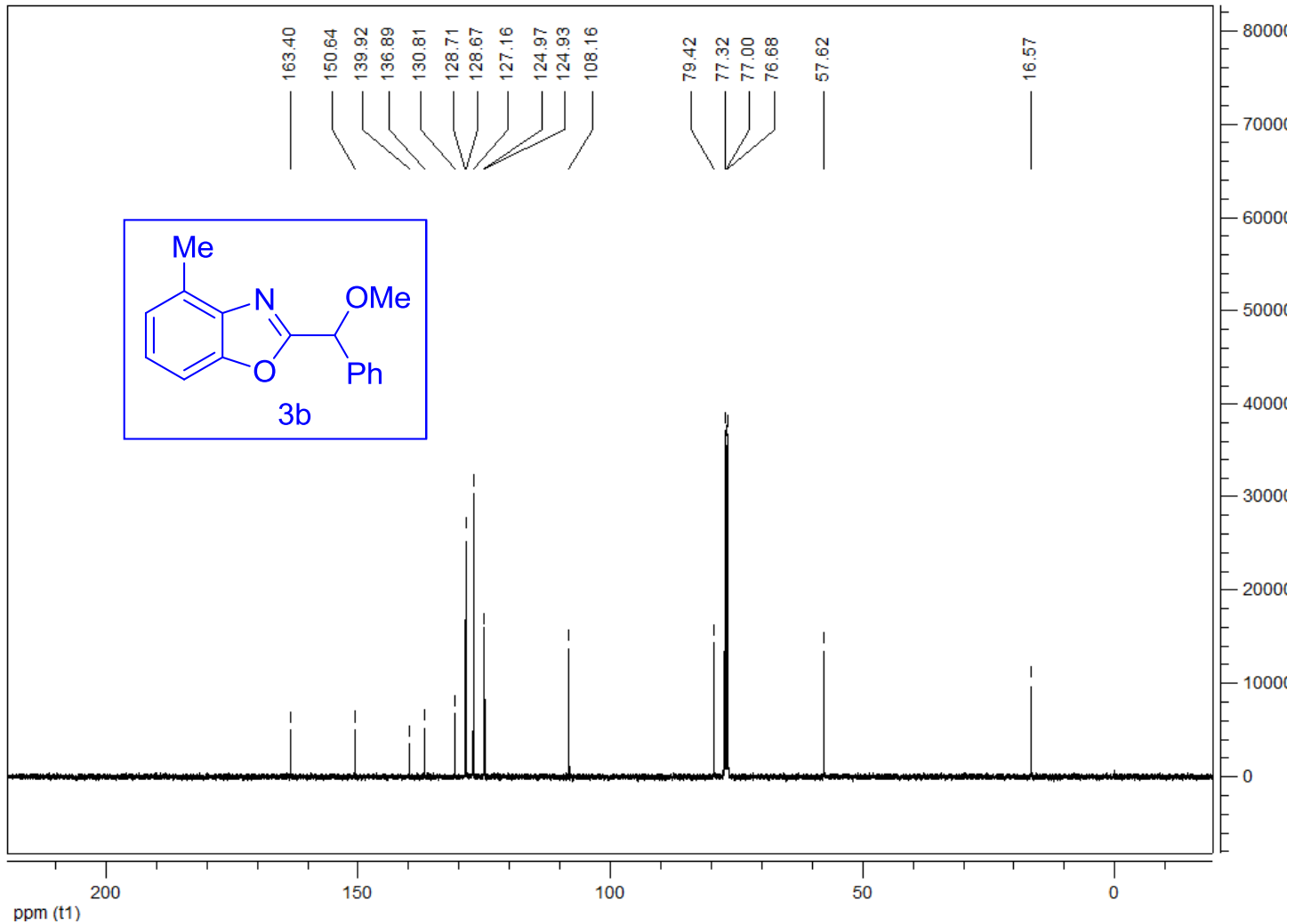


Figure S53. ${ }^{1}$ H NMR of 2-(methoxy(phenyl)methyl)-5-methylbenzo[d]oxazole (3c)

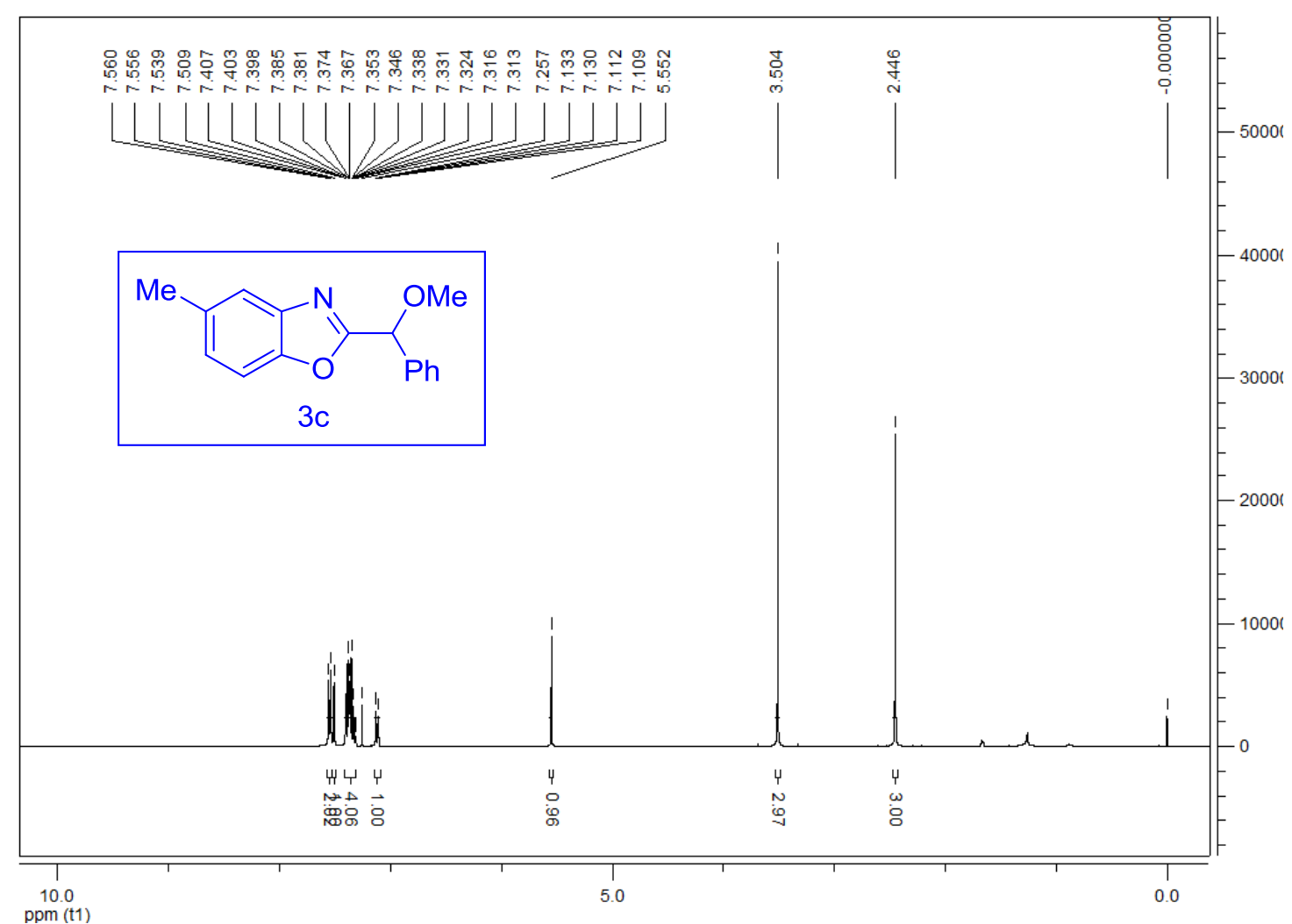

Figure S54. ${ }^{13} \mathrm{C}$ NMR of 2-(methoxy(phenyl)methyl)-5-methylbenzo[d]oxazole (3c)

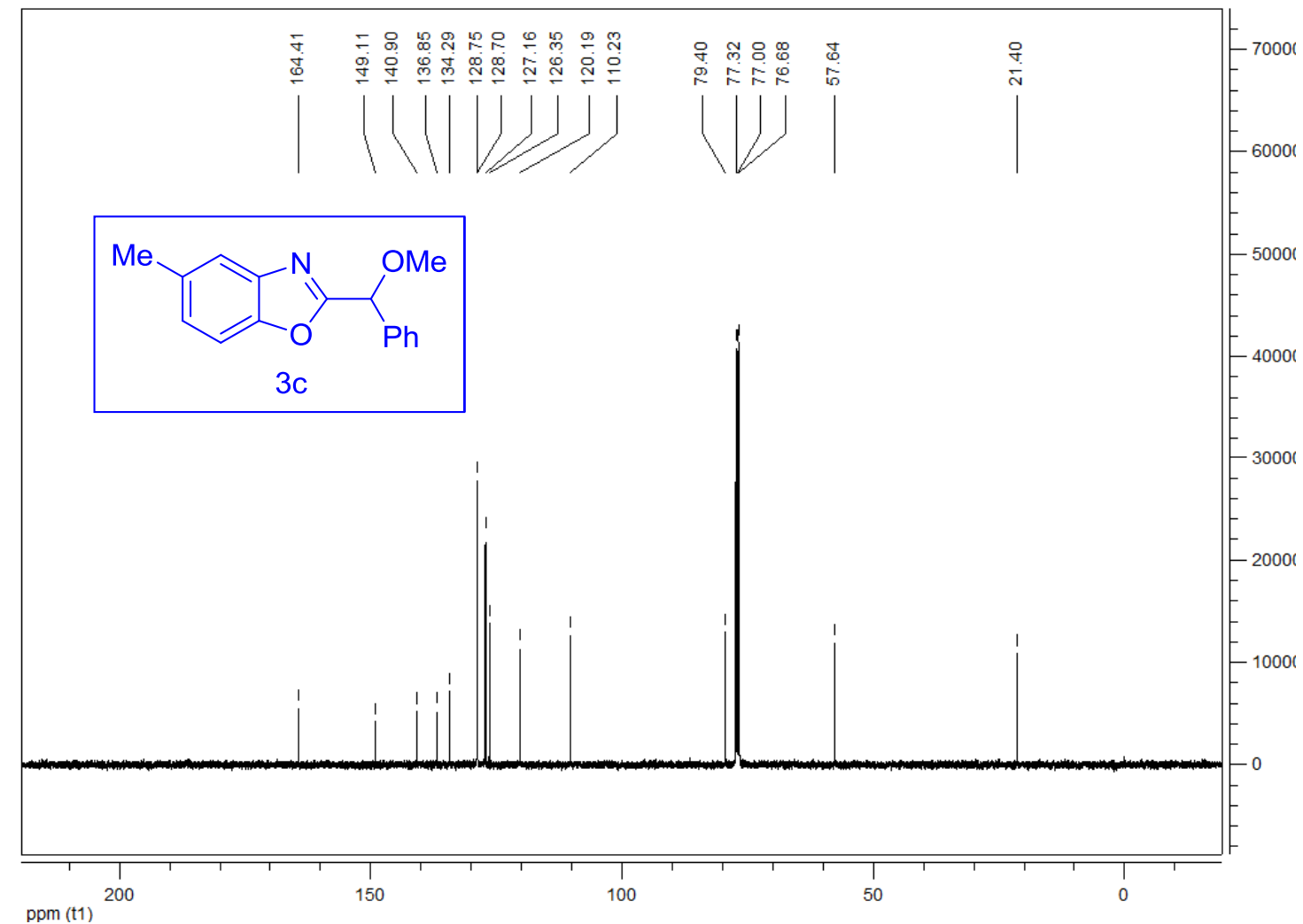


Figure S55. ${ }^{1}$ H NMR of 5-tert-butyl-2-(methoxy(phenyl)methyl)benzo[d]oxazole (3d)

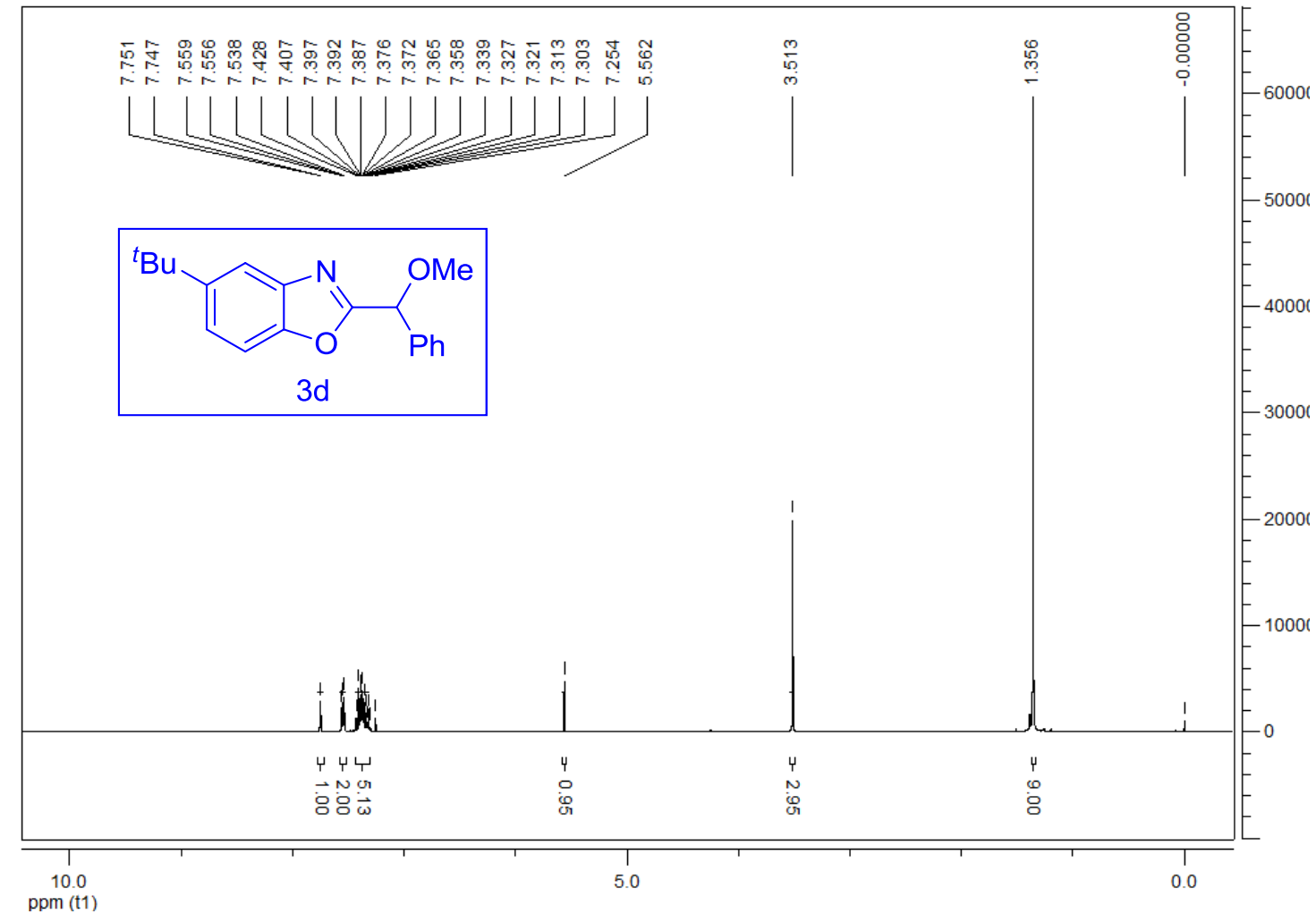

Figure S56. ${ }^{13} \mathrm{C}$ NMR of 5-tert-butyl-2-(methoxy(phenyl)methyl)benzo[d]oxazole (3d)

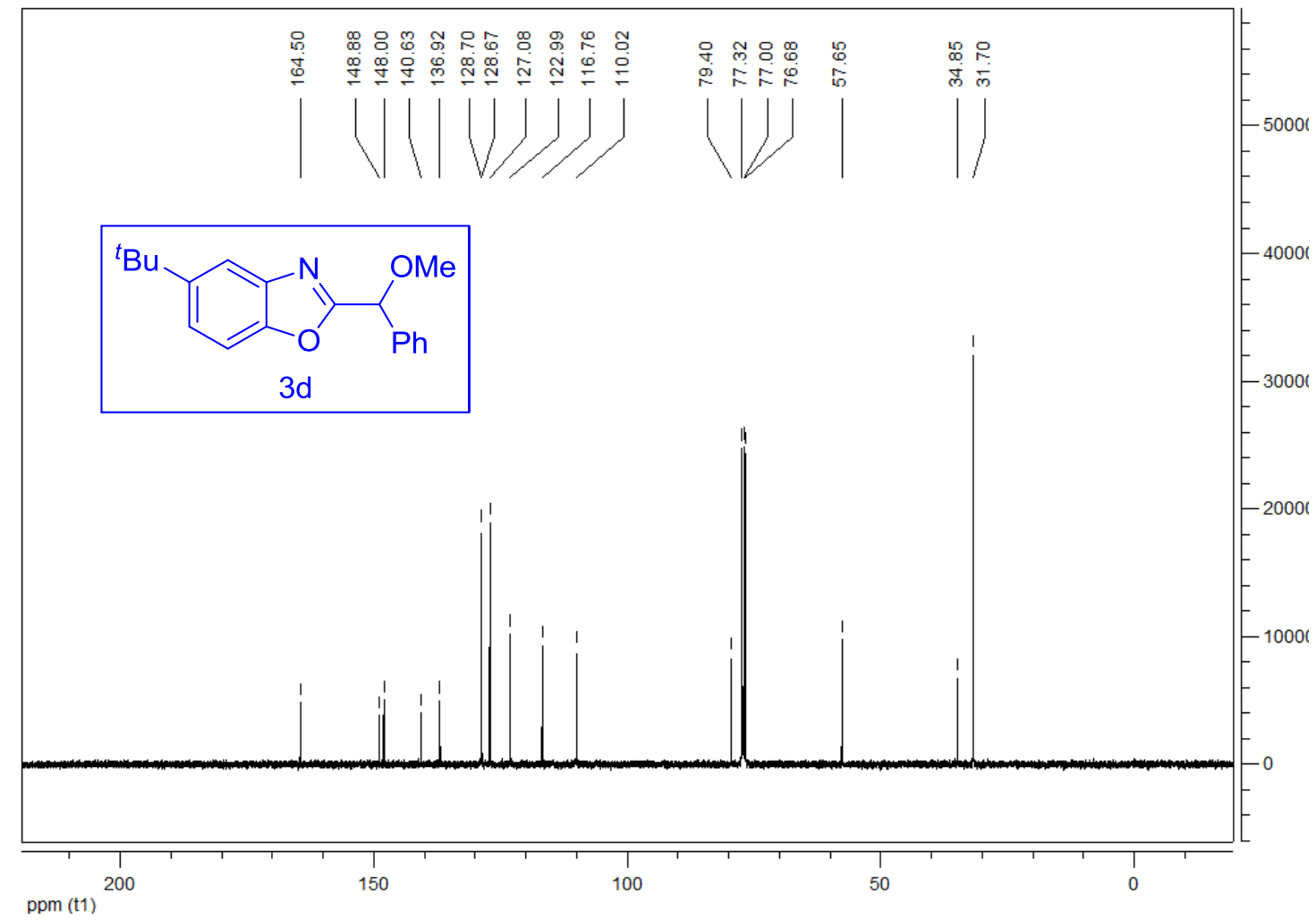


Figure S57. ${ }^{1}$ H NMR of 5-methoxy-2-(methoxy(phenyl)methyl)benzo[d]oxazole (3e)

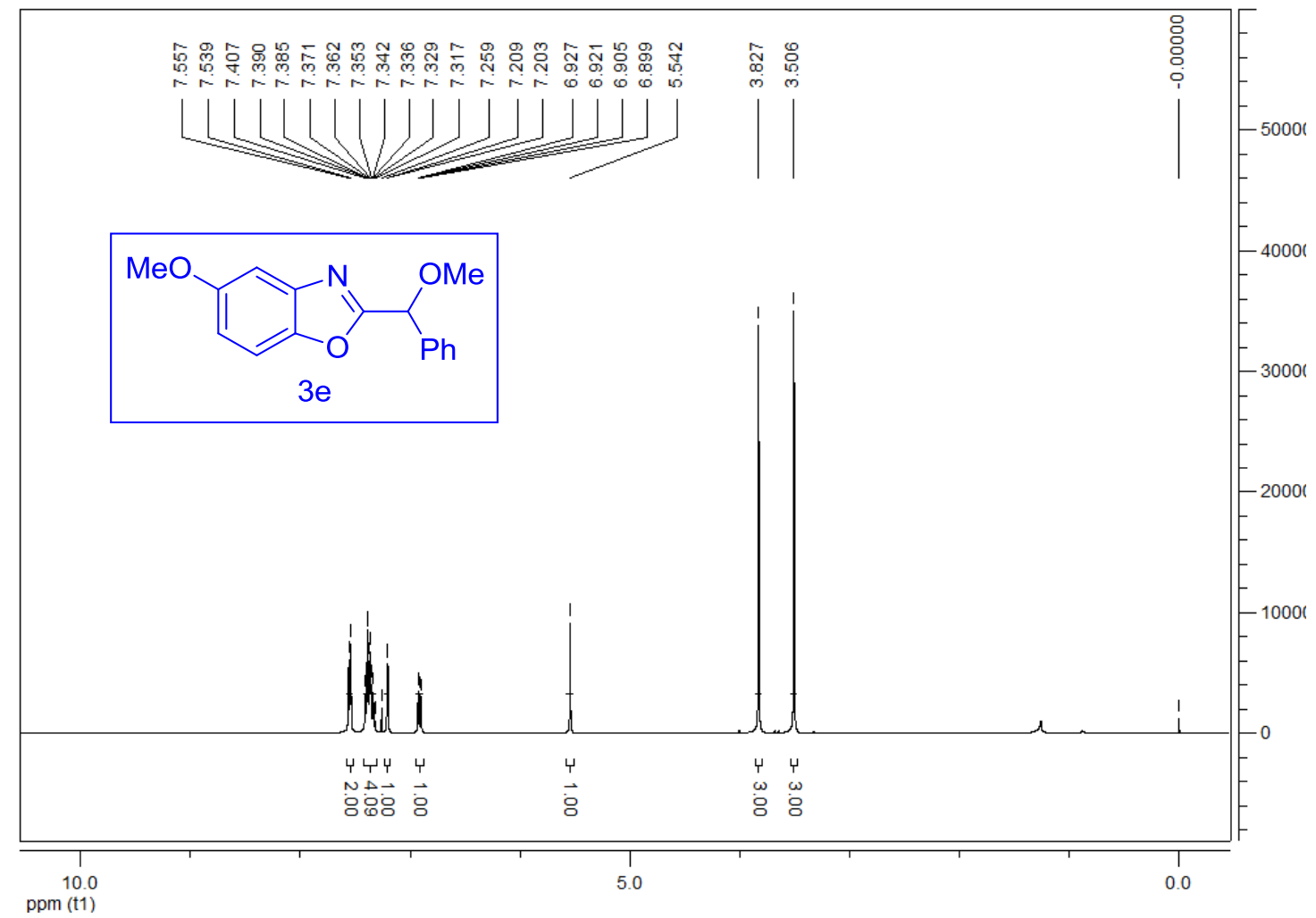

Figure S58. ${ }^{13} \mathrm{C}$ NMR of 5-methoxy-2-(methoxy(phenyl)methyl)benzo[d]oxazole (3e)

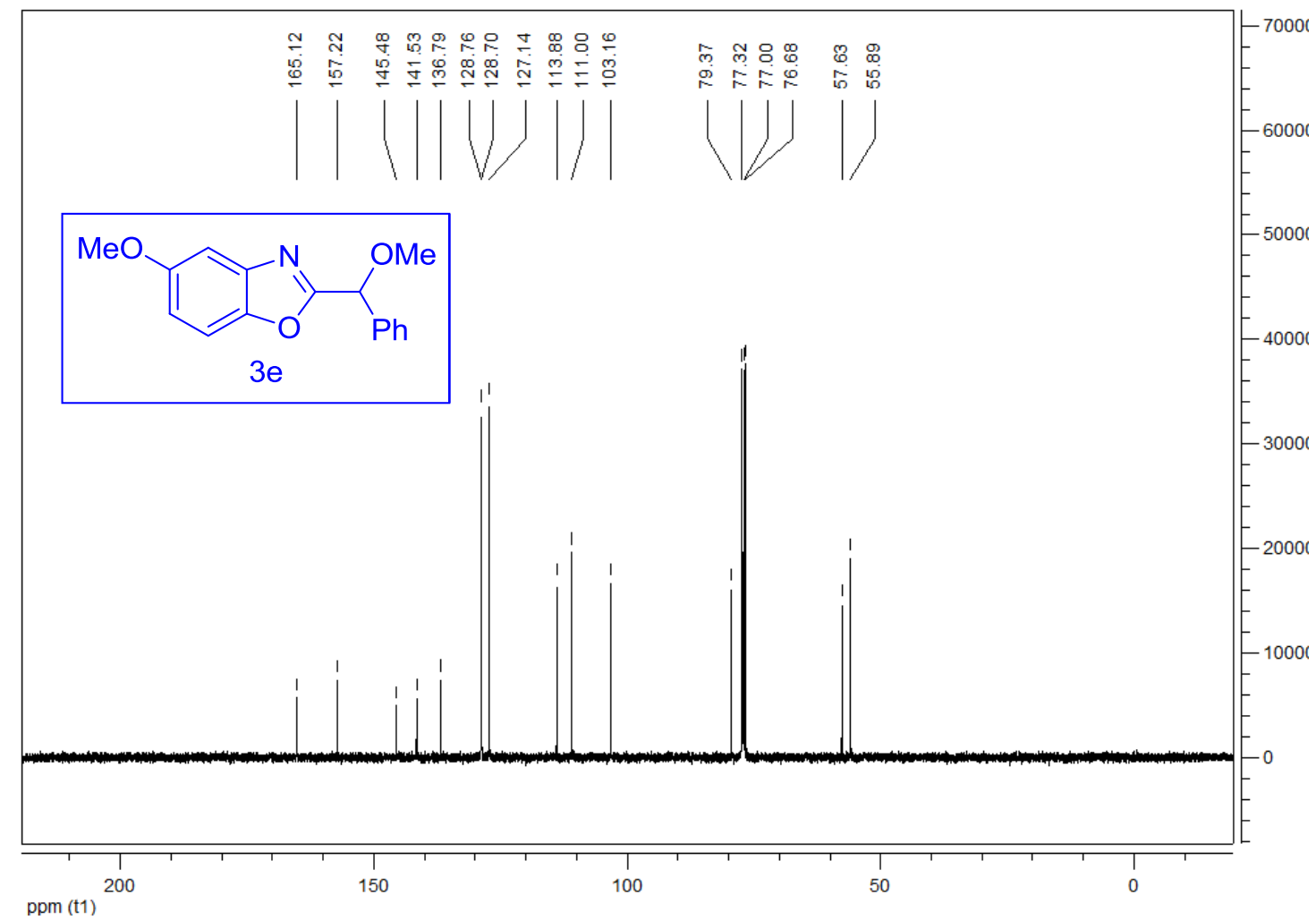


Figure S59. ${ }^{1}$ H NMR of 5-fluoro-2-(methoxy(phenyl)methyl)benzo[d]oxazole (3f)

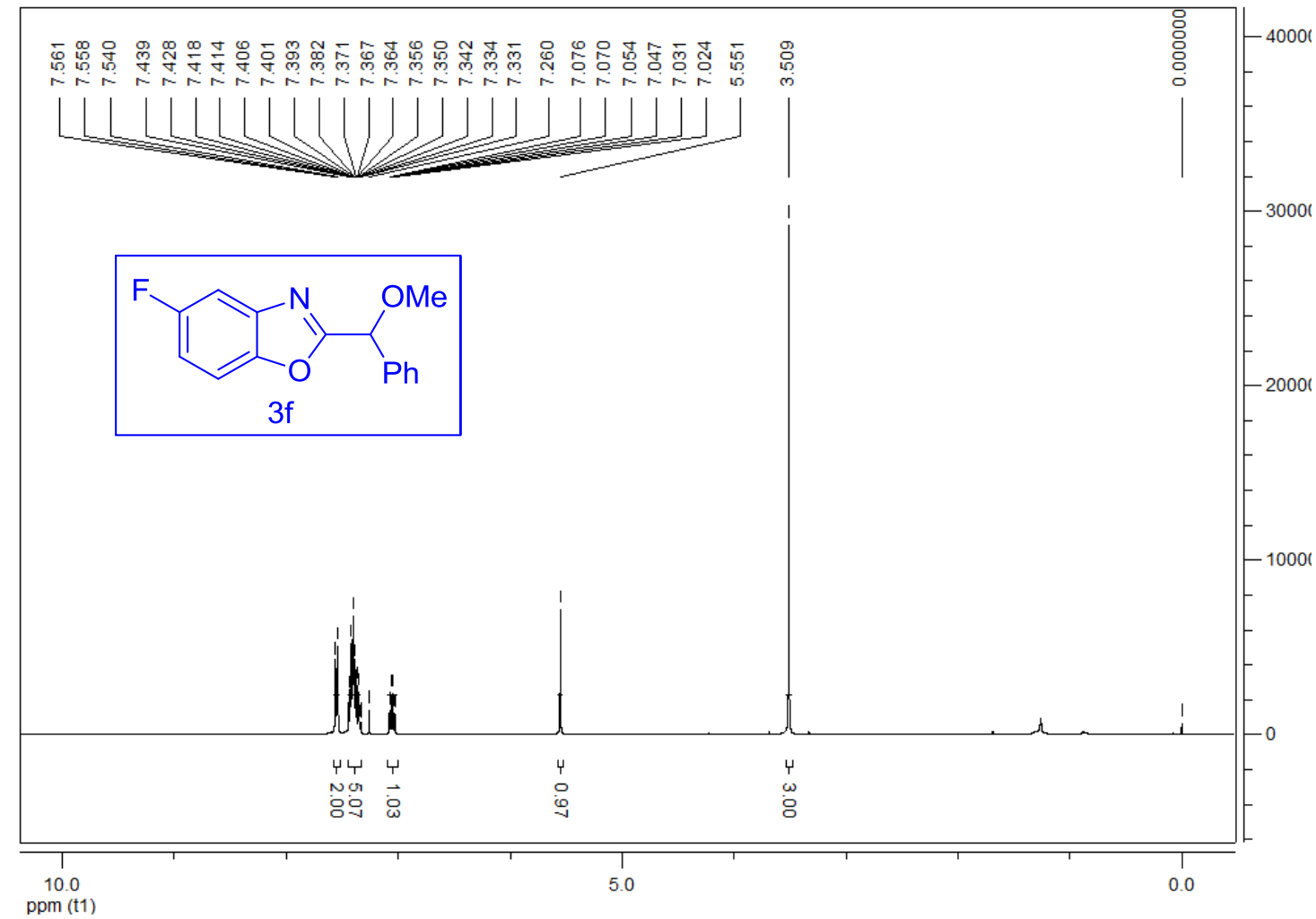

Figure S60. ${ }^{13} \mathrm{C}$ NMR of 5-fluoro-2-(methoxy(phenyl)methyl)benzo[d]oxazole (3f)

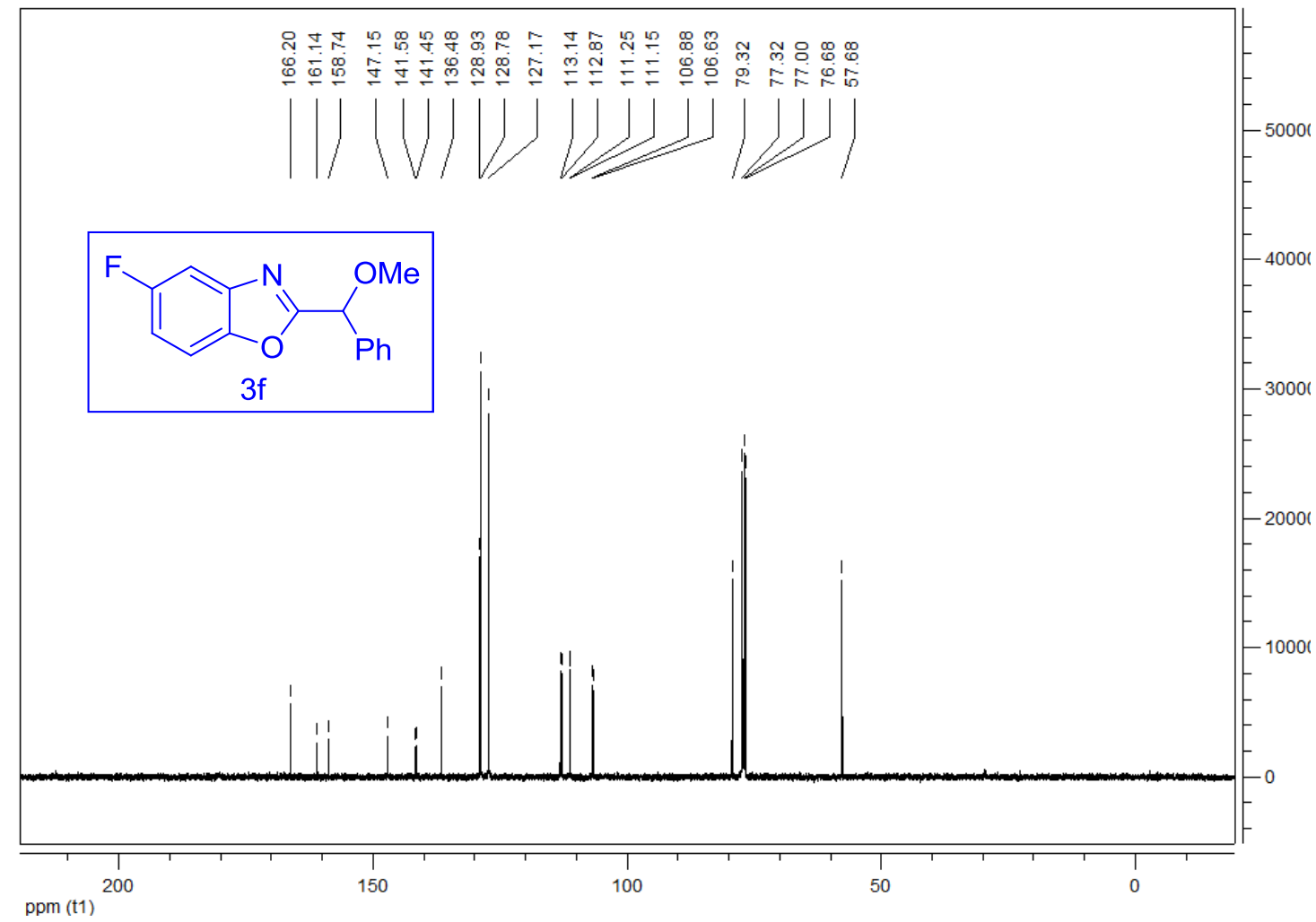


Figure S61. ${ }^{1}$ H NMR of 5-chloro-2-(methoxy(phenyl)methyl)benzo[d]oxazole (3g)

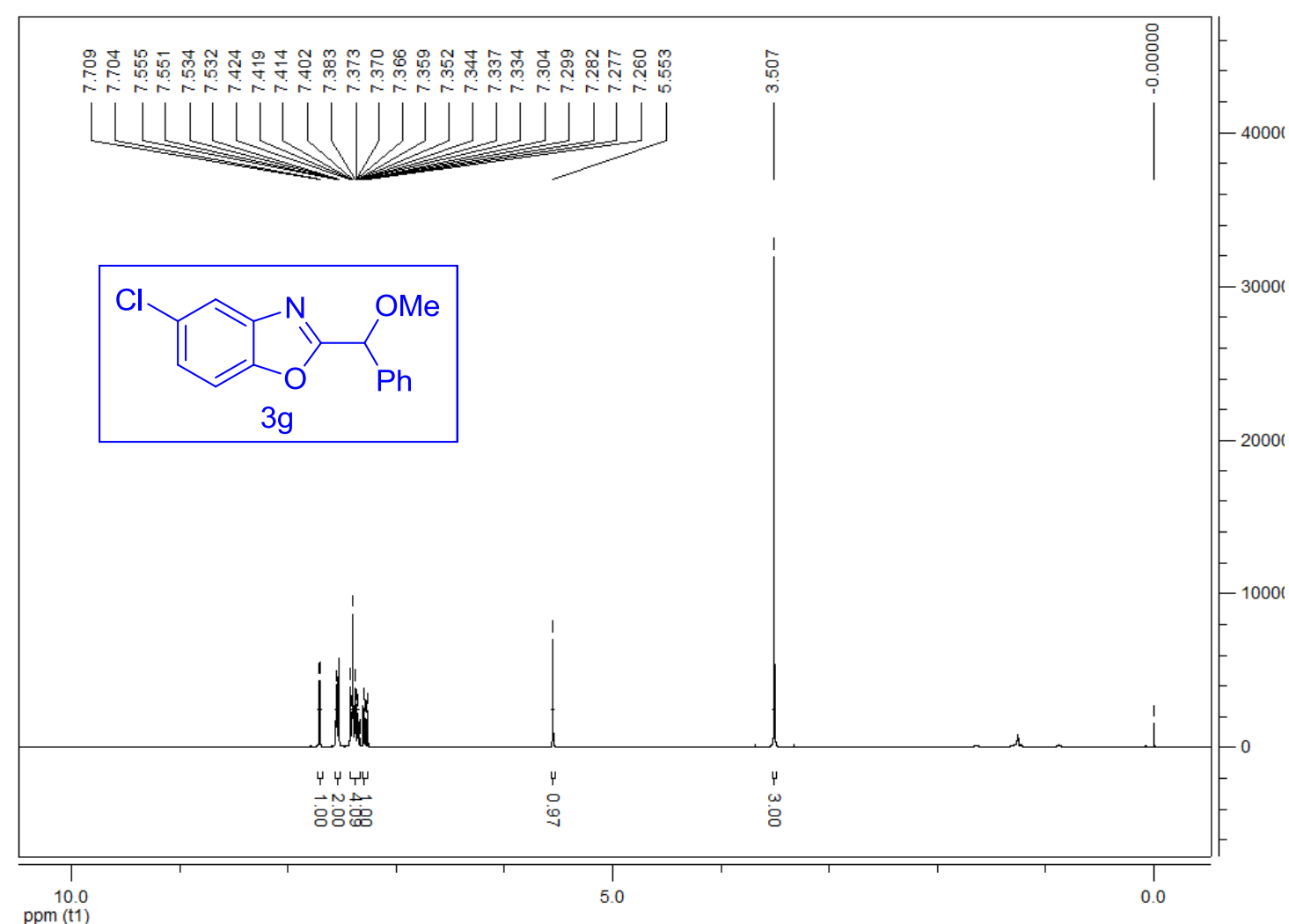

Figure S62. ${ }^{13} \mathrm{C}$ NMR of 5-chloro-2-(methoxy(phenyl)methyl)benzo[d]oxazole (3g)

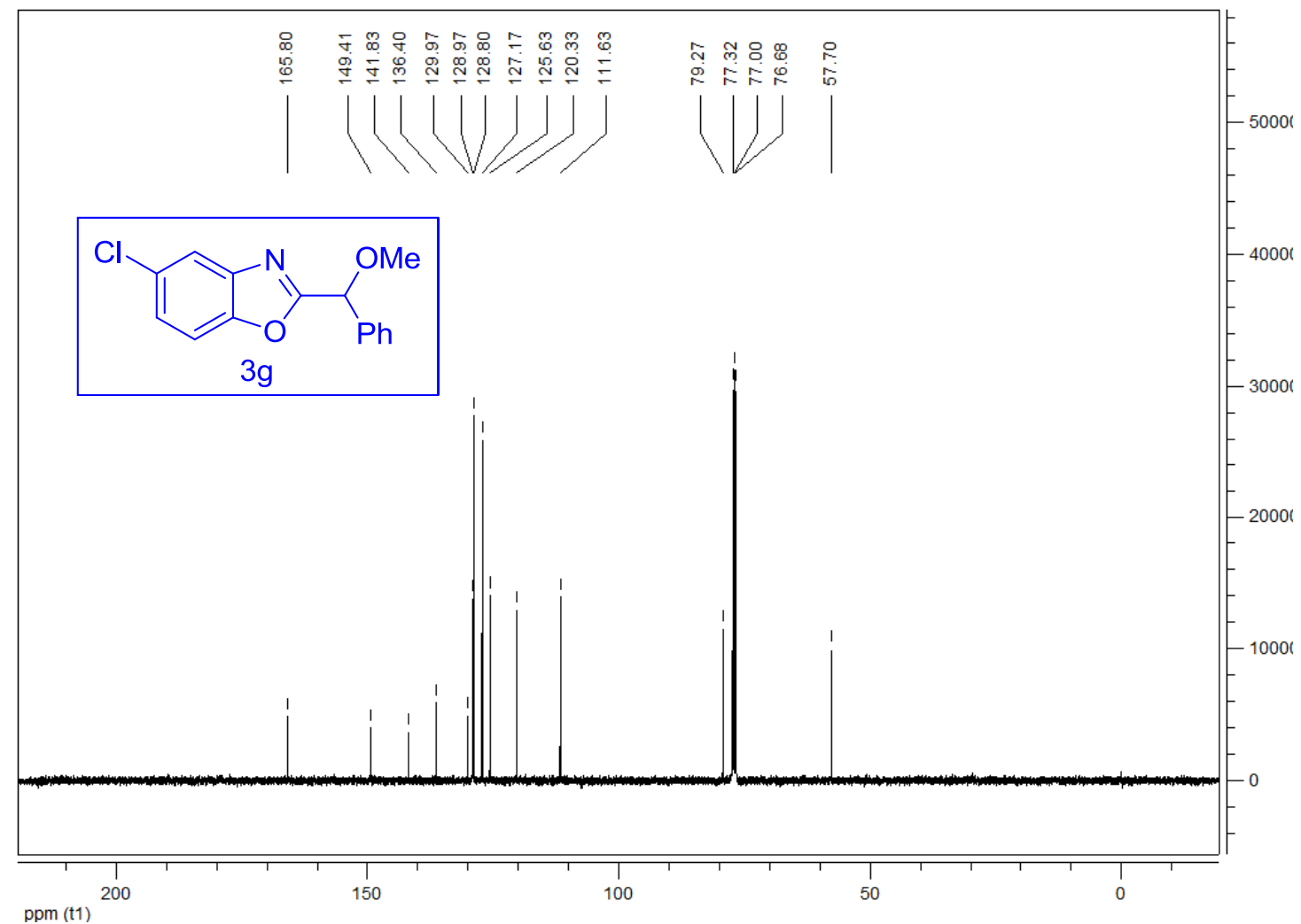


Figure S63. ${ }^{1}$ H NMR of 2-(methoxy(phenyl)methyl)-6-methylbenzo[d]oxazole (3h)

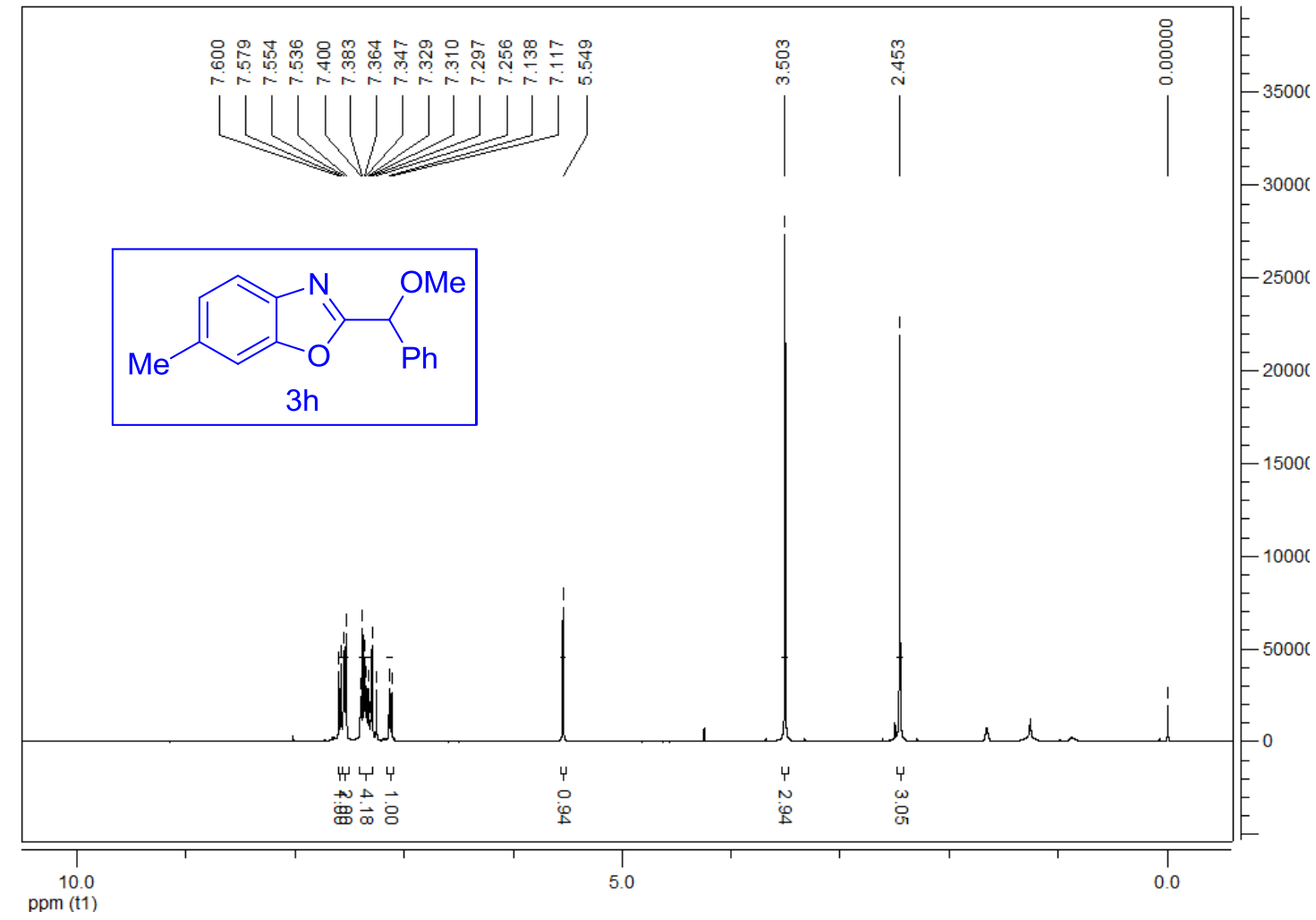

Figure S64. ${ }^{13} \mathrm{C}$ NMR of 2-(methoxy(phenyl)methyl)-6-methylbenzo[d]oxazole (3h)

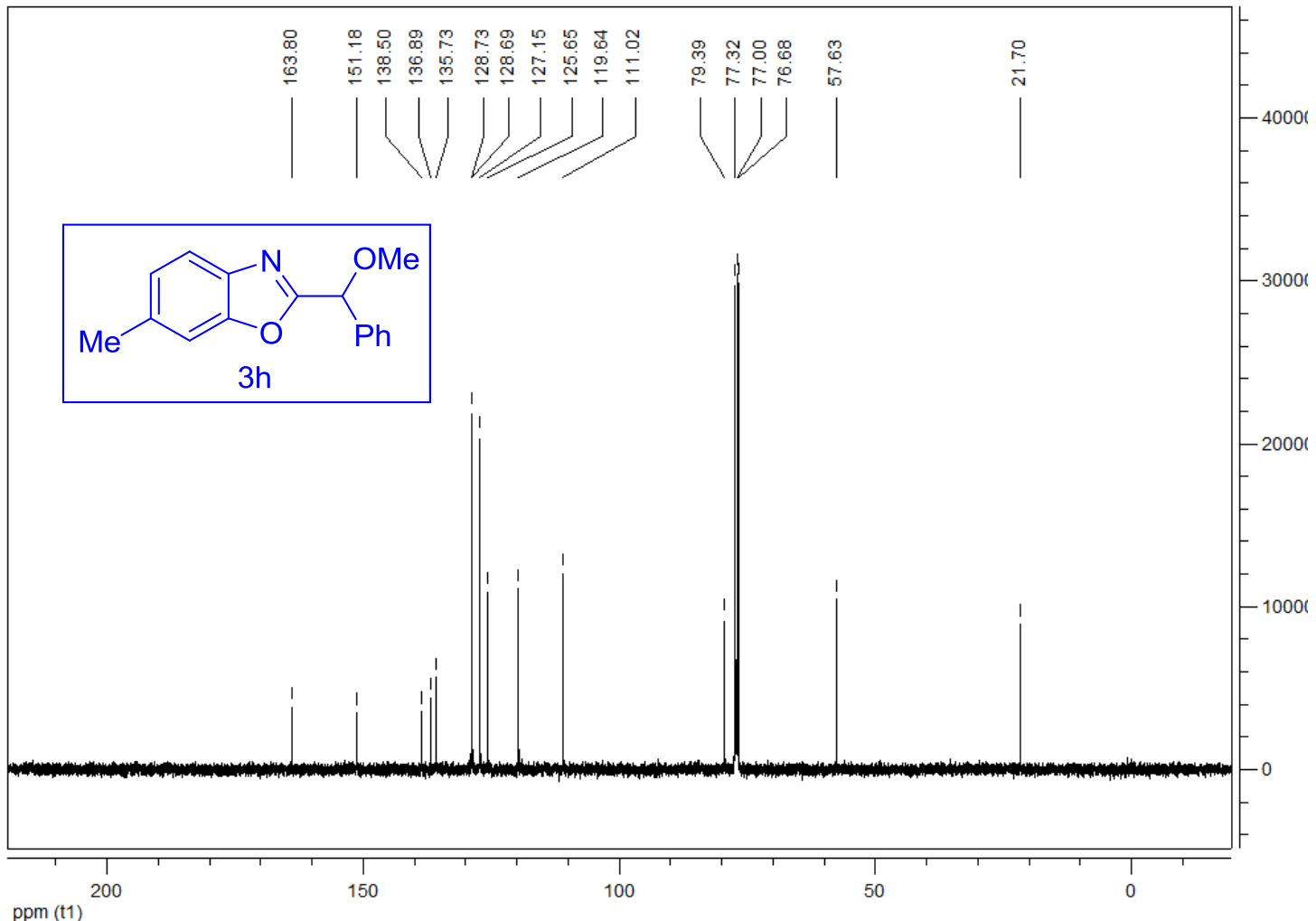


Figure S65. ${ }^{1}$ H NMR of 6-chloro-2-(methoxy(phenyl)methyl)benzo[d]oxazole (3i)

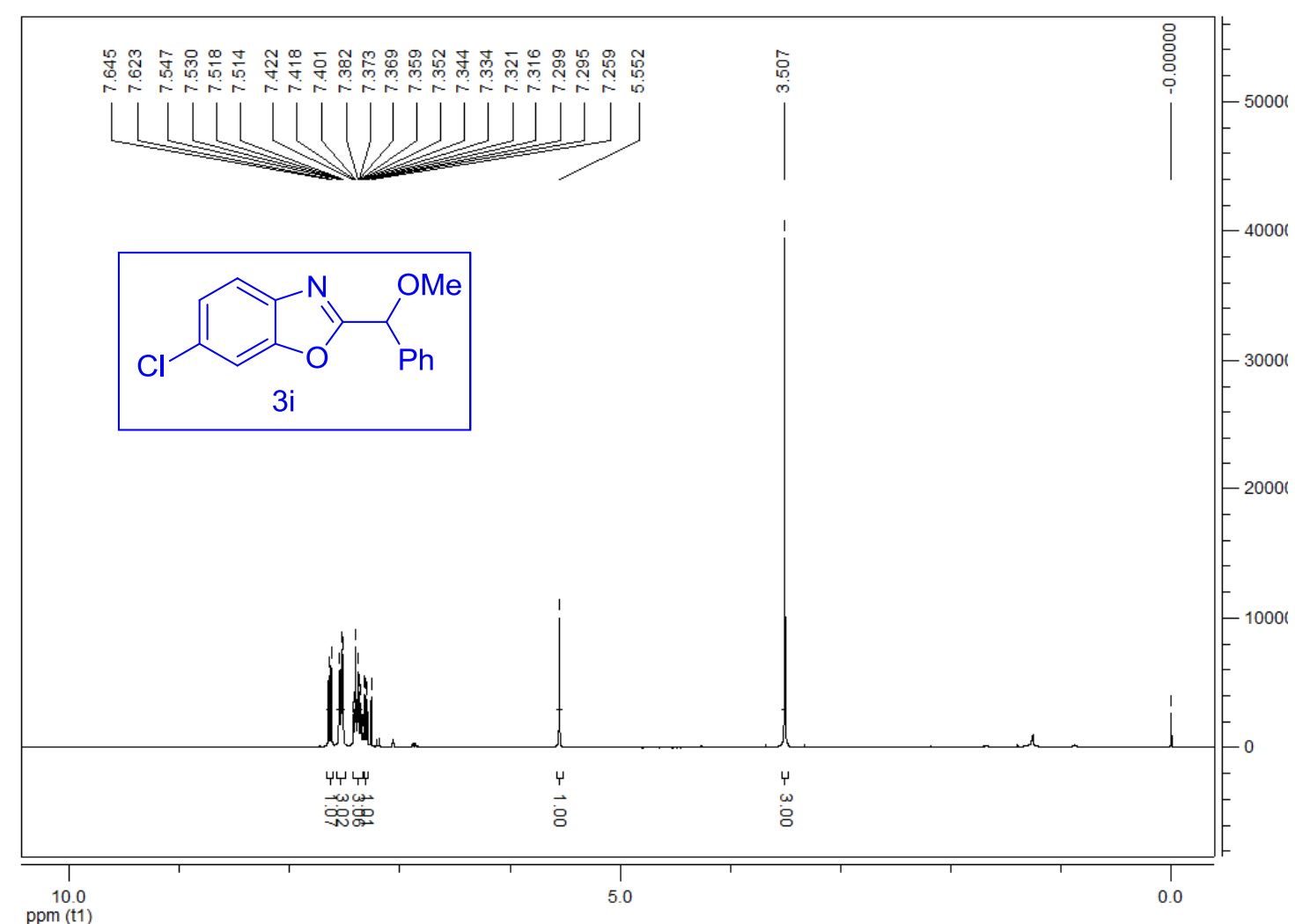

Figure S66. ${ }^{13} \mathrm{C}$ NMR of 6-chloro-2-(methoxy(phenyl)methyl)benzo[d]oxazole (3i)

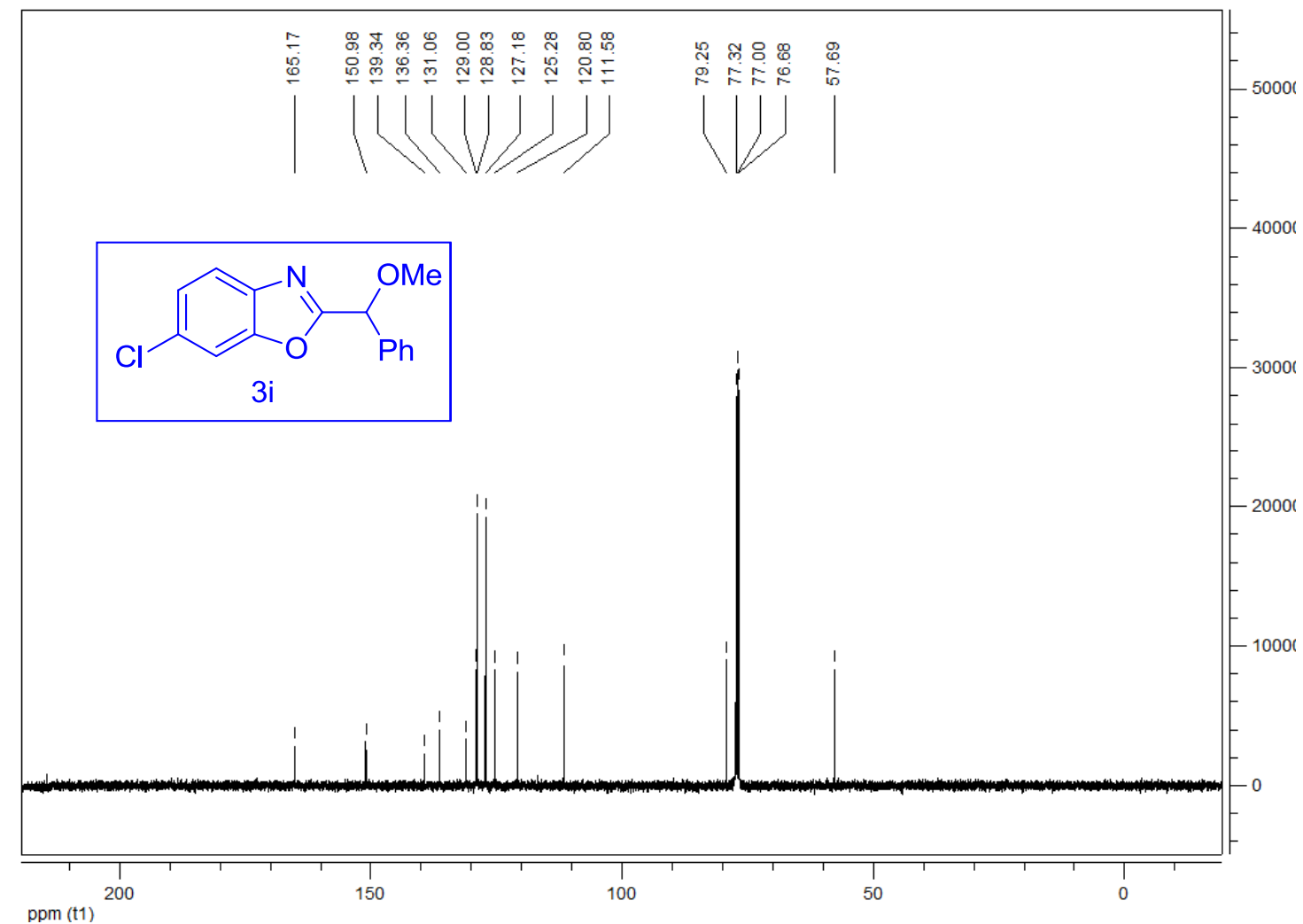


Figure S67. ${ }^{1}$ H NMR of 2-(methoxy(phenyl)methyl)-7-methylbenzo[d]oxazole (3j)

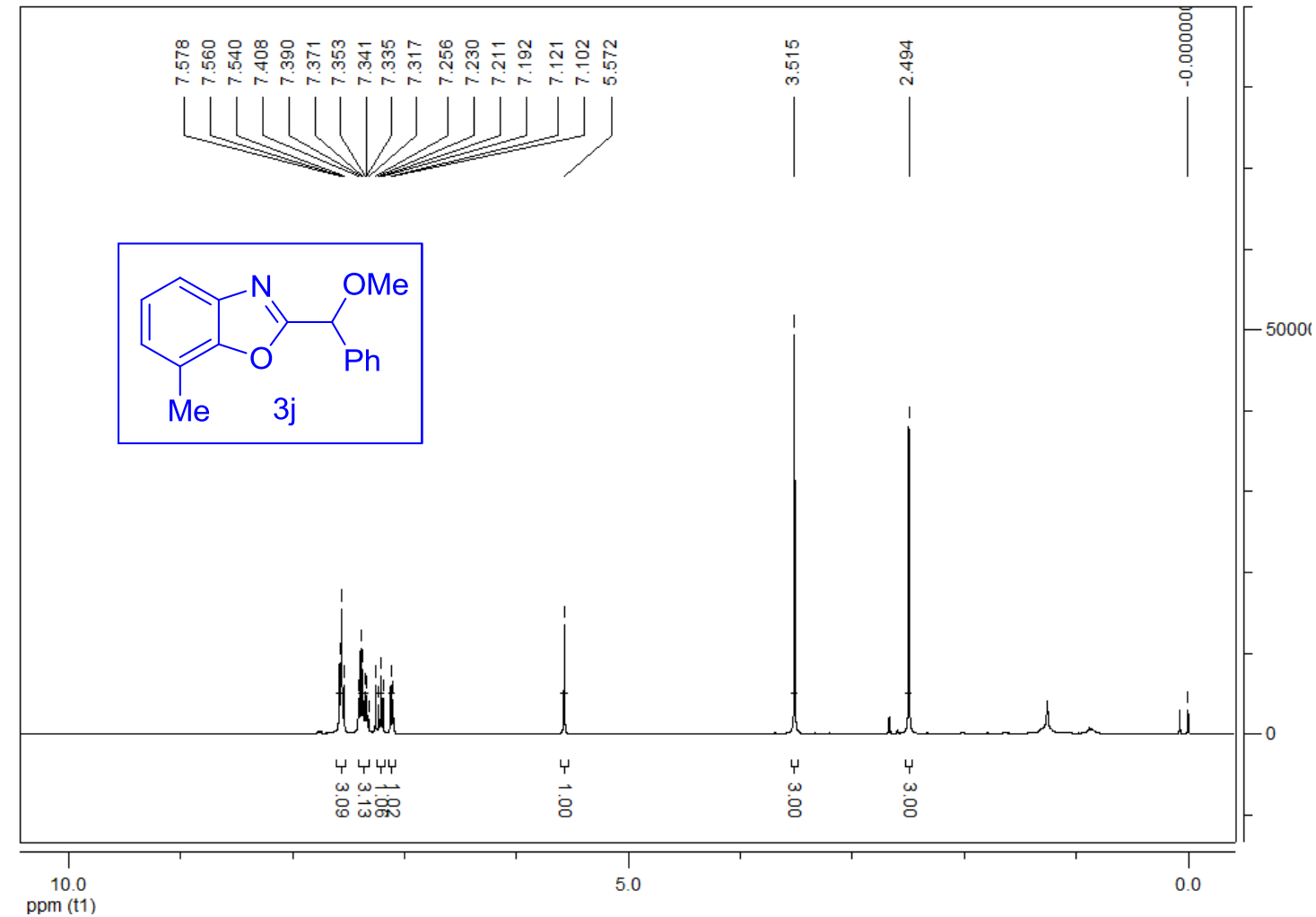

Figure S68. ${ }^{13} \mathrm{C}$ NMR of 2-(methoxy(phenyl)methyl)-7-methylbenzo[d]oxazole (3j)

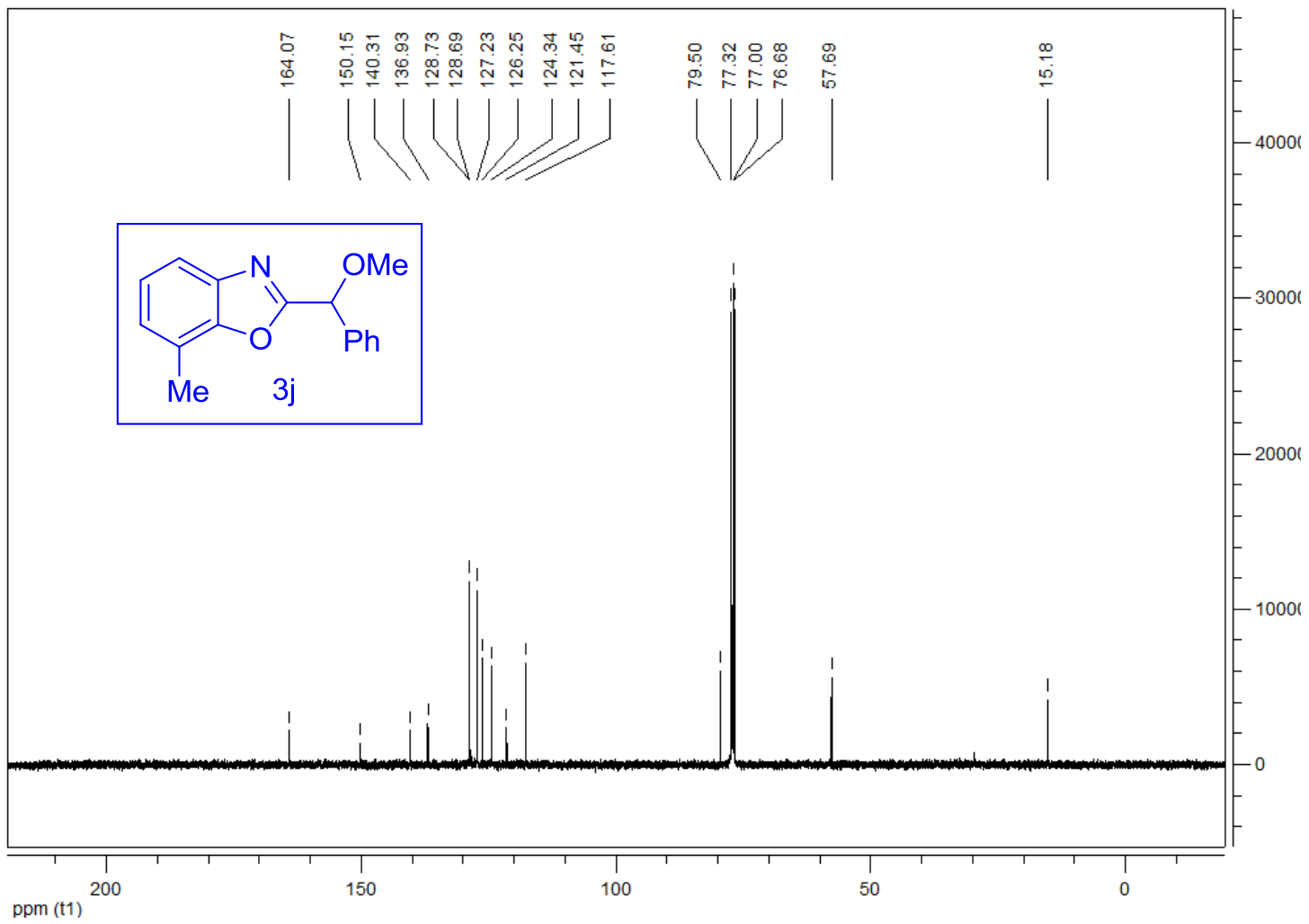


Figure S69. ${ }^{1}$ H NMR of 7-bromo-2-(methoxy(phenyl)methyl)benzo[d]oxazole (3k)

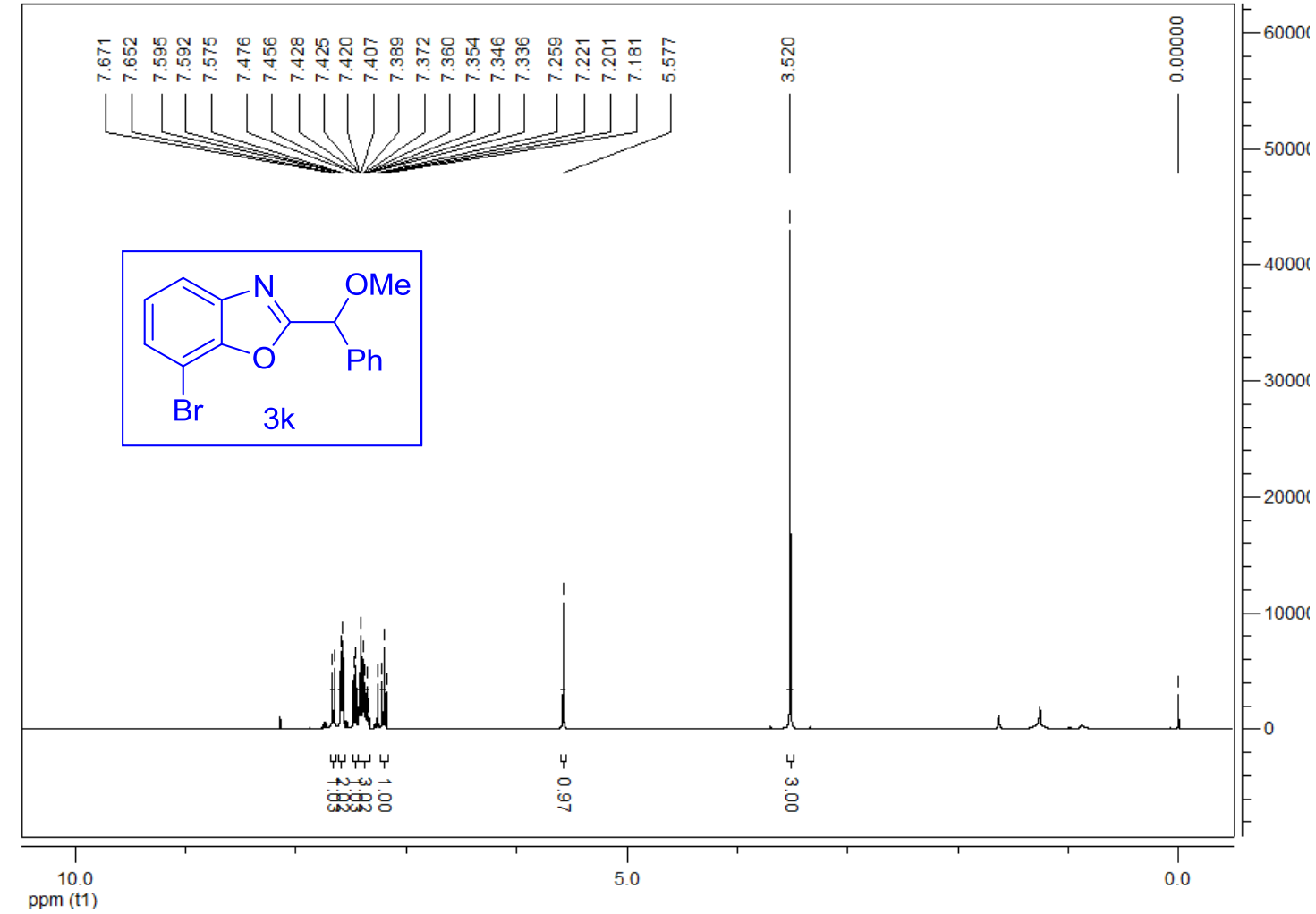

Figure S70. ${ }^{13} \mathrm{C}$ NMR of 7-bromo-2-(methoxy(phenyl)methyl)benzo[d]oxazole (3k)

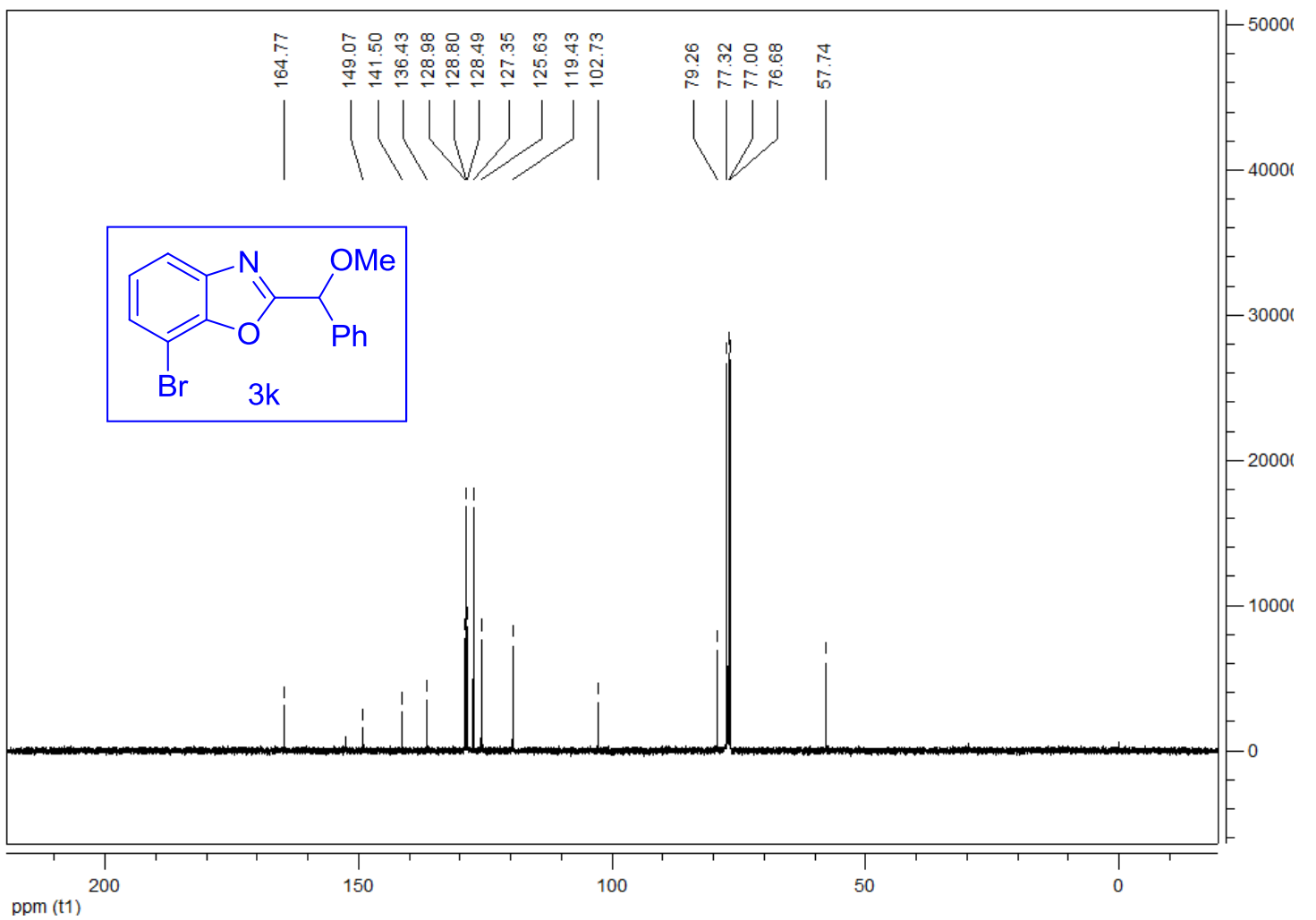


Figure S71. ${ }^{1}$ H NMR of 2-(methoxy(o-tolyl)methyl)benzo[d]oxazole (4a)

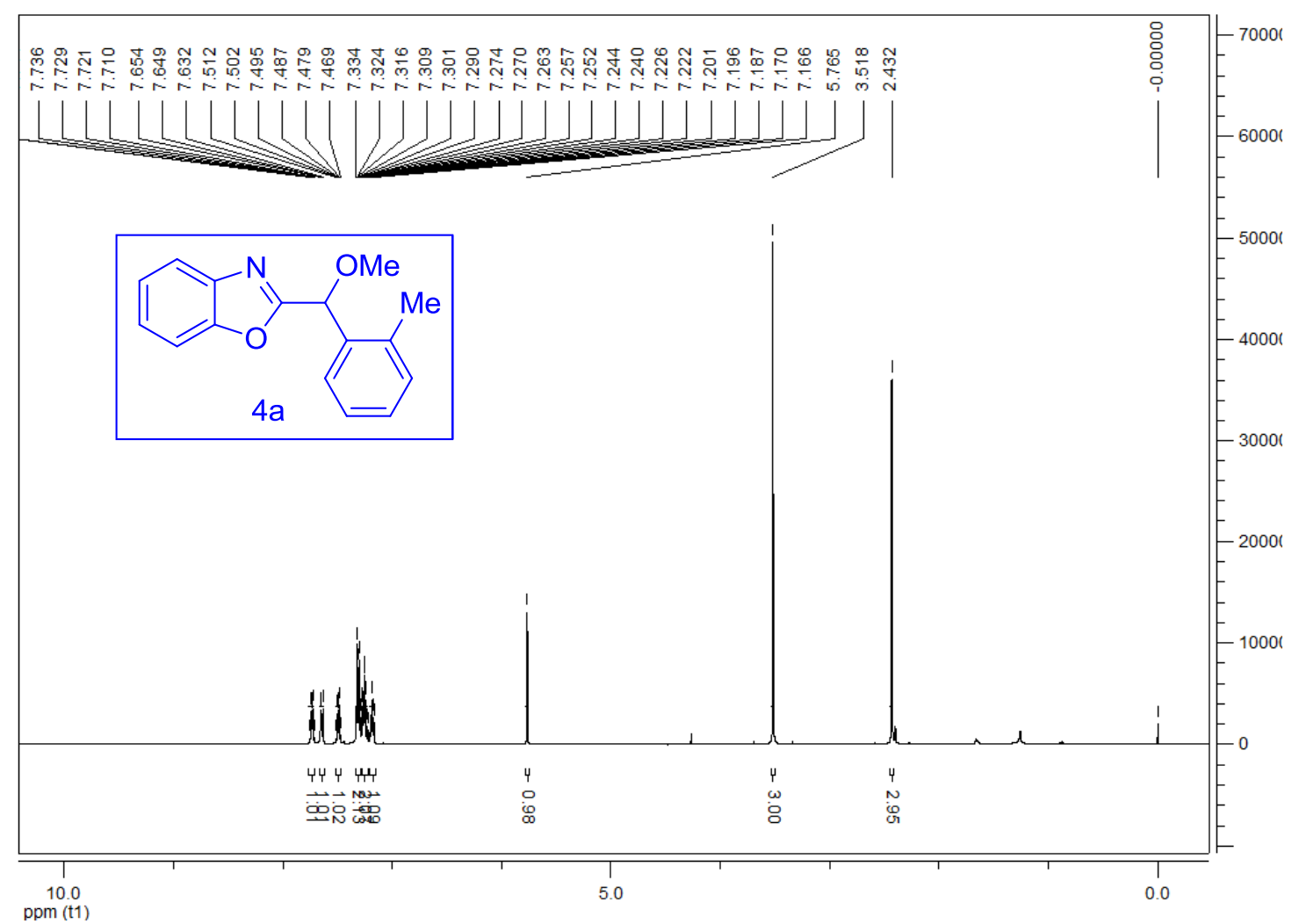

Figure S72. ${ }^{13} \mathrm{C}$ NMR of 2-(methoxy(o-tolyl)methyl)benzo[d]oxazole (4a)

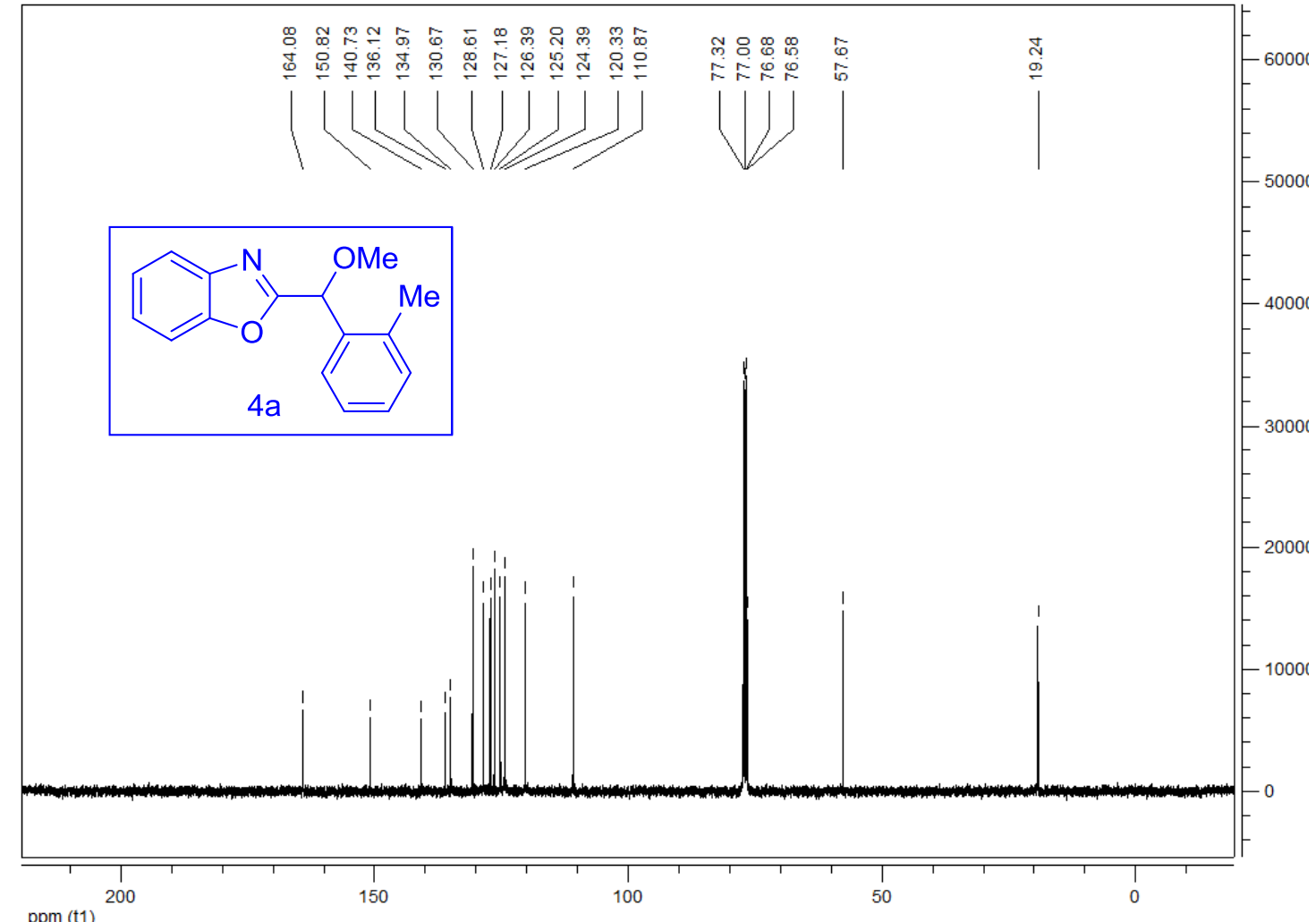


Figure S73. ${ }^{1} \mathrm{H}$ NMR of 2-(methoxy(2-methoxyphenyl)methyl)benzo[d]oxazole (4b)

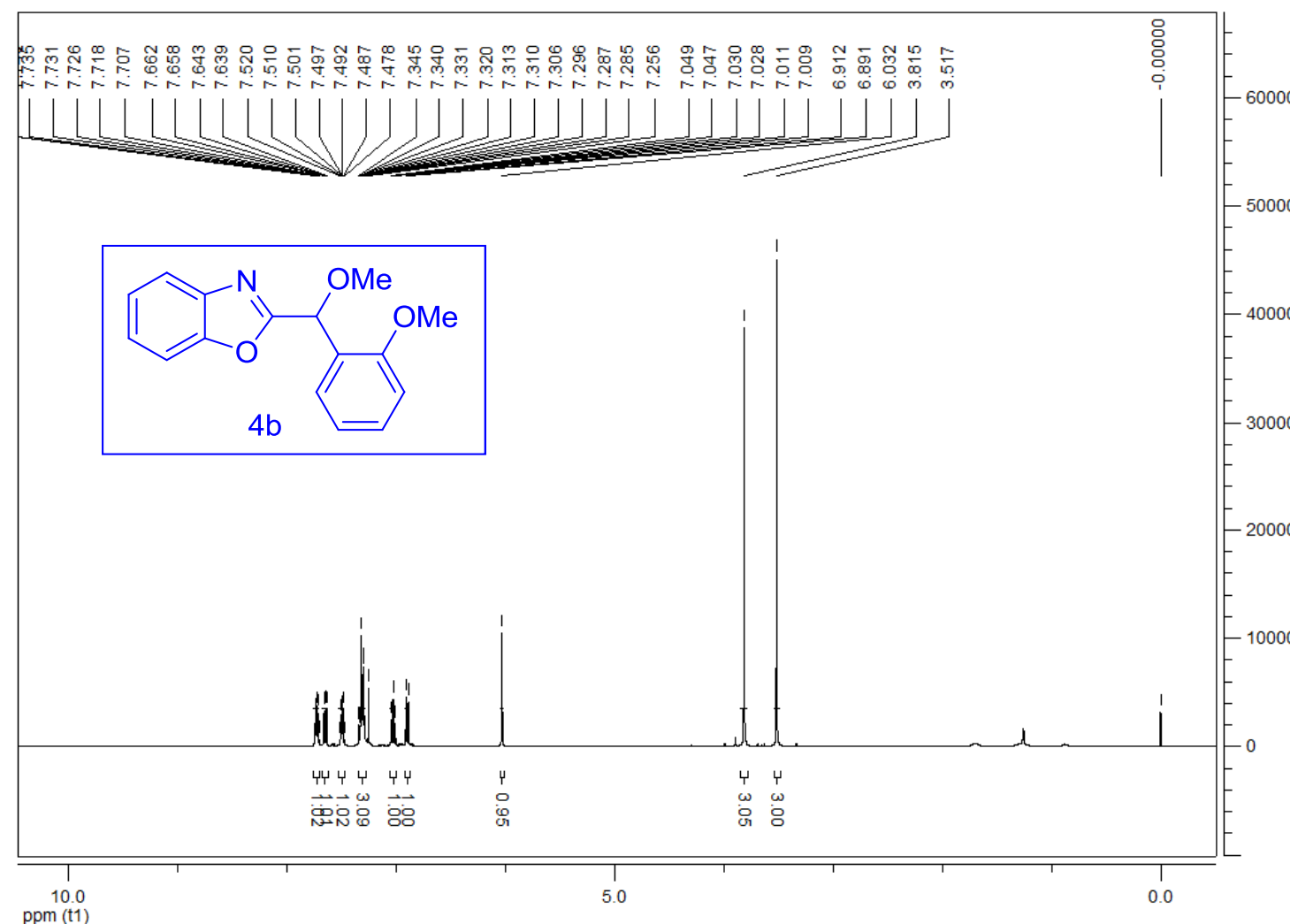

Figure S74. ${ }^{13} \mathrm{C}$ NMR of 2-(methoxy(2-methoxyphenyl)methyl)benzo[d]oxazole (4b)

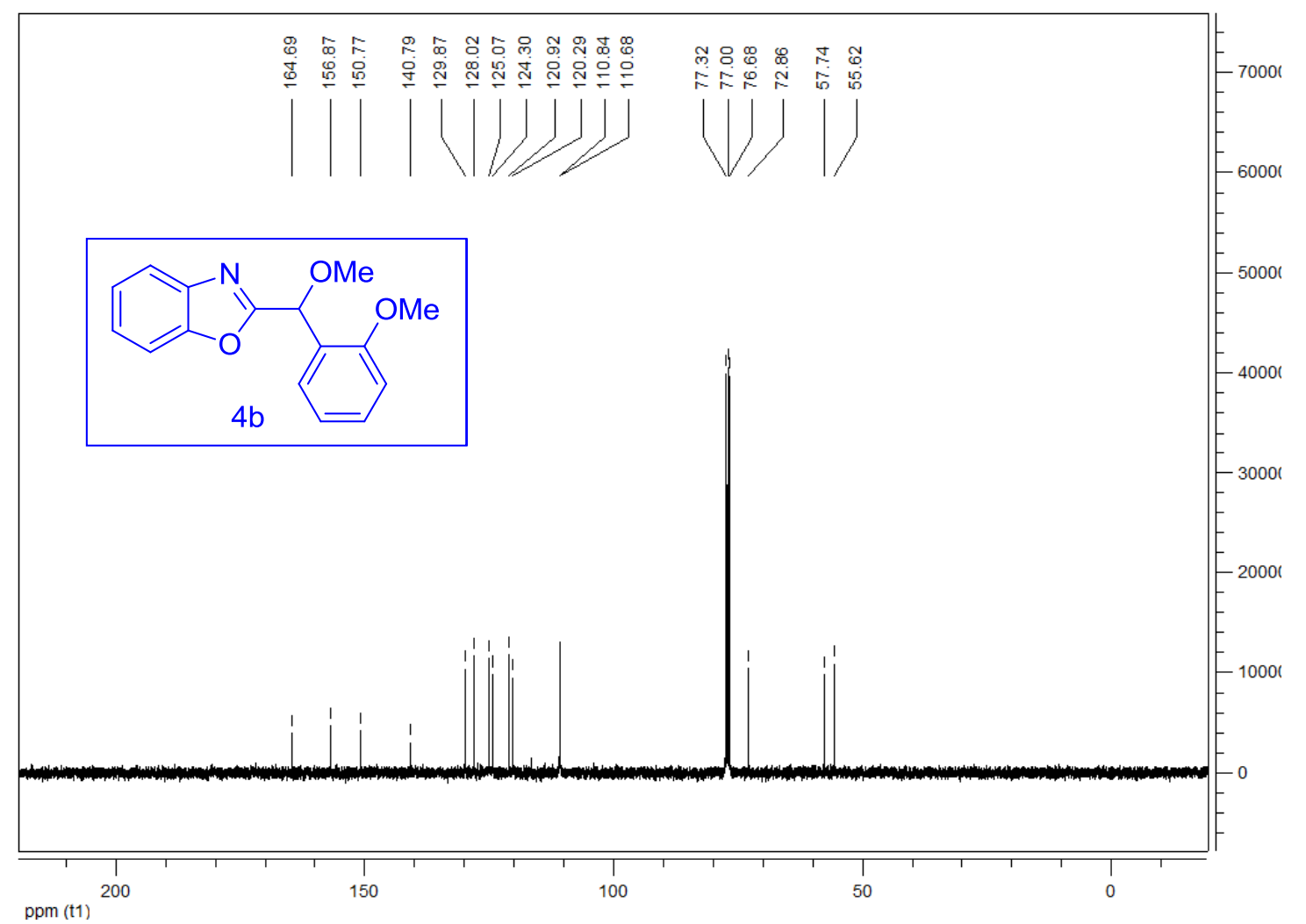


Figure S75. ${ }^{1} \mathrm{H}$ NMR of 2-((3-chlorophenyl)(methoxy)methyl)benzo[d]oxazole (4c)

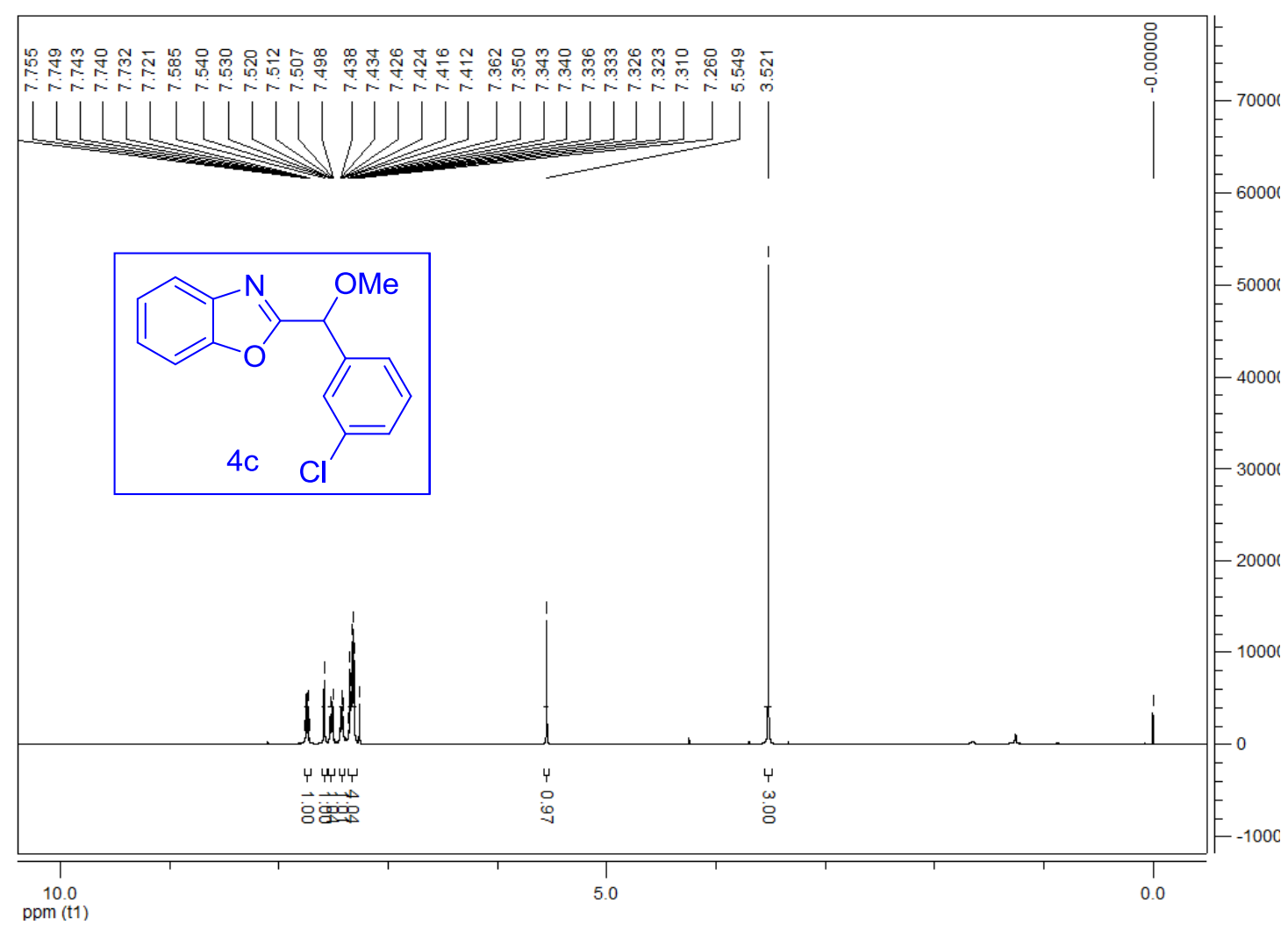

Figure S76. ${ }^{13} \mathrm{C}$ NMR of 2-((3-chlorophenyl)(methoxy)methyl)benzo[d]oxazole (4c)

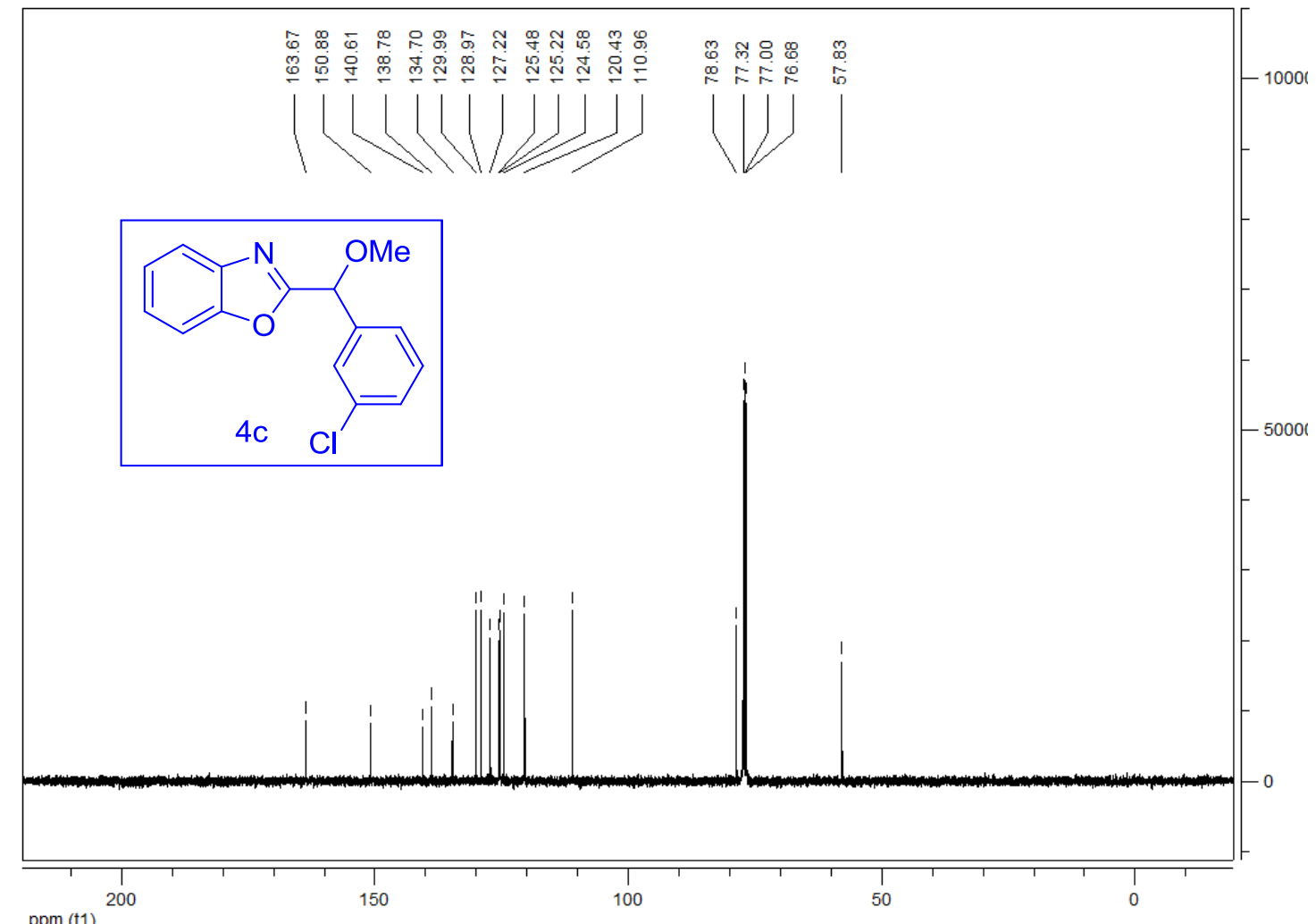


Figure S77. ${ }^{1} \mathrm{H}$ NMR of 2-(methoxy(3-methoxyphenyl)methyl)benzo[d]oxazole (4d)

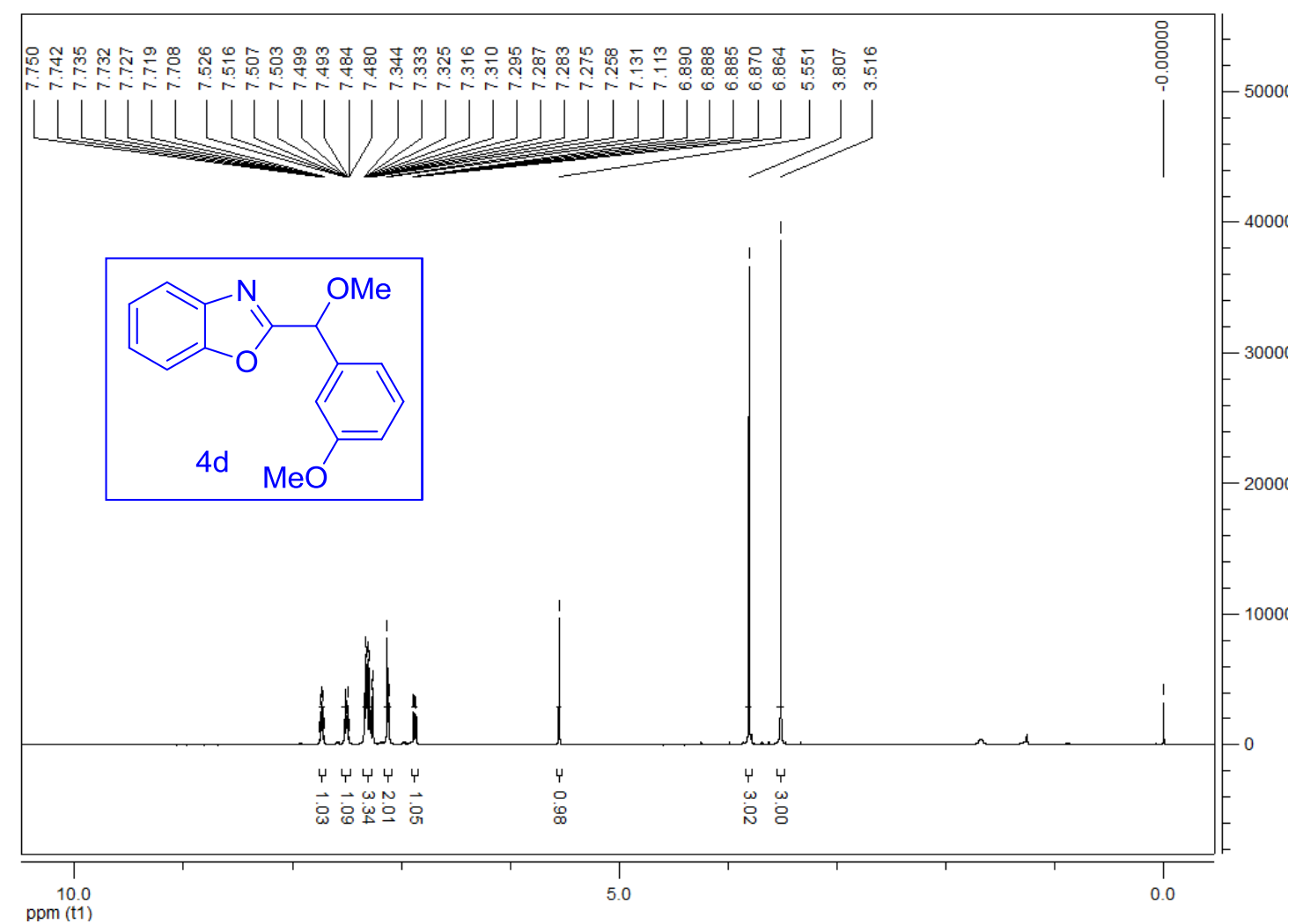

Figure S78. ${ }^{13} \mathrm{C}$ NMR of 2-(methoxy(3-methoxyphenyl)methyl)benzo[d]oxazole (4d)

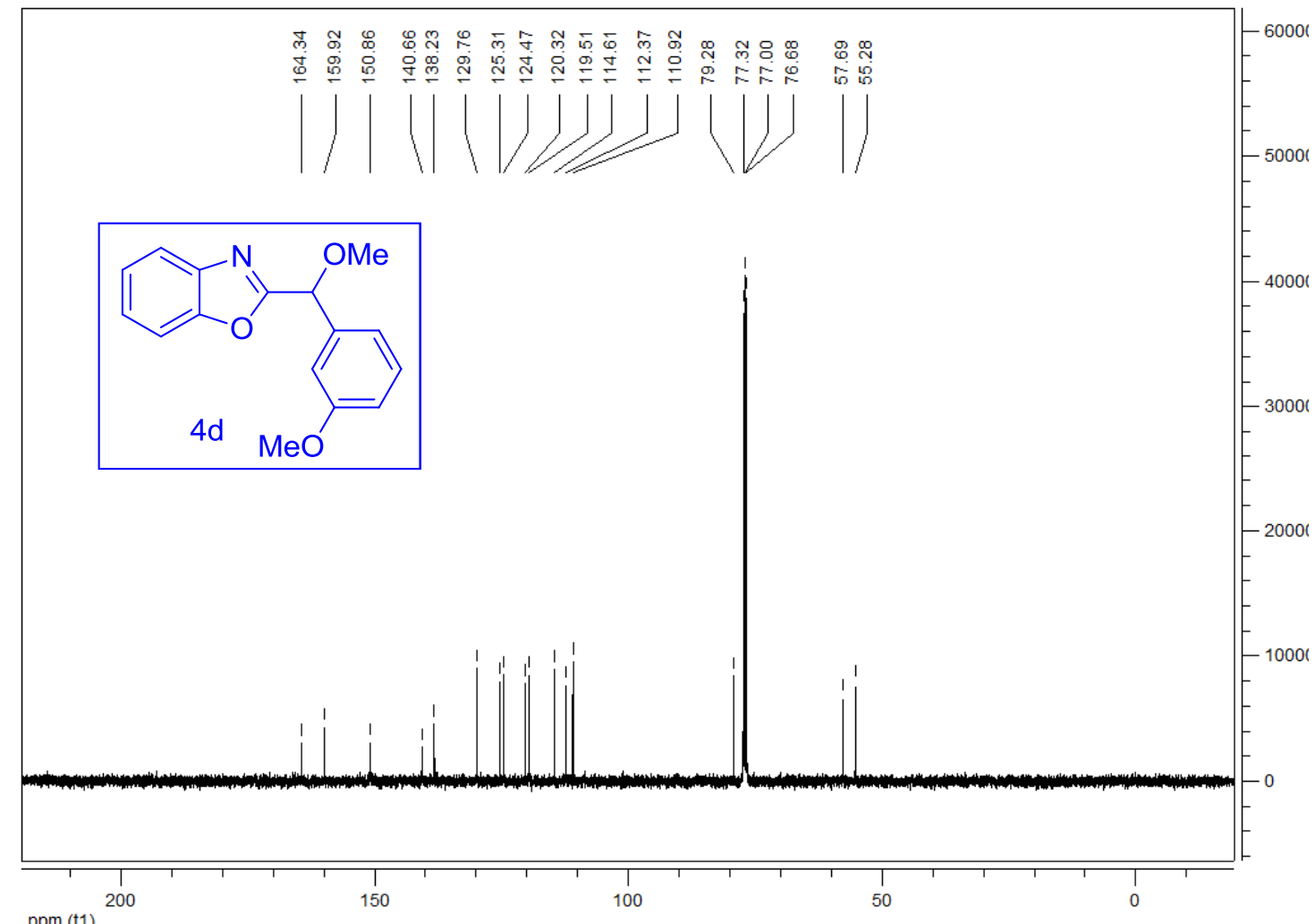


Figure S79. ${ }^{1}$ H NMR of 2-(methoxy(p-tolyl)methyl)benzo[d]oxazole (4e)

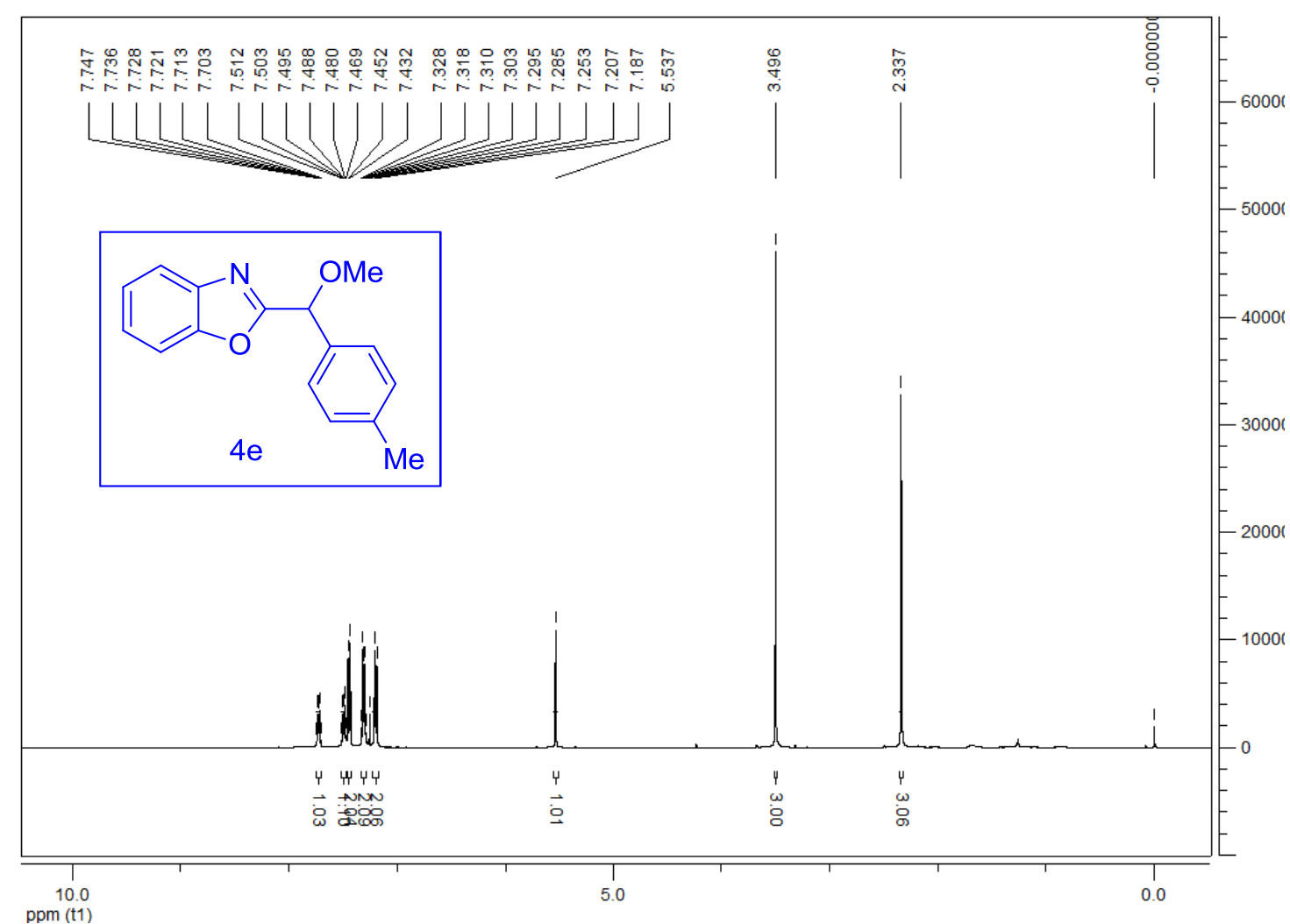

Figure S80. ${ }^{13} \mathrm{C}$ NMR of 2-(methoxy(p-tolyl)methyl)benzo[d]oxazole (4e)

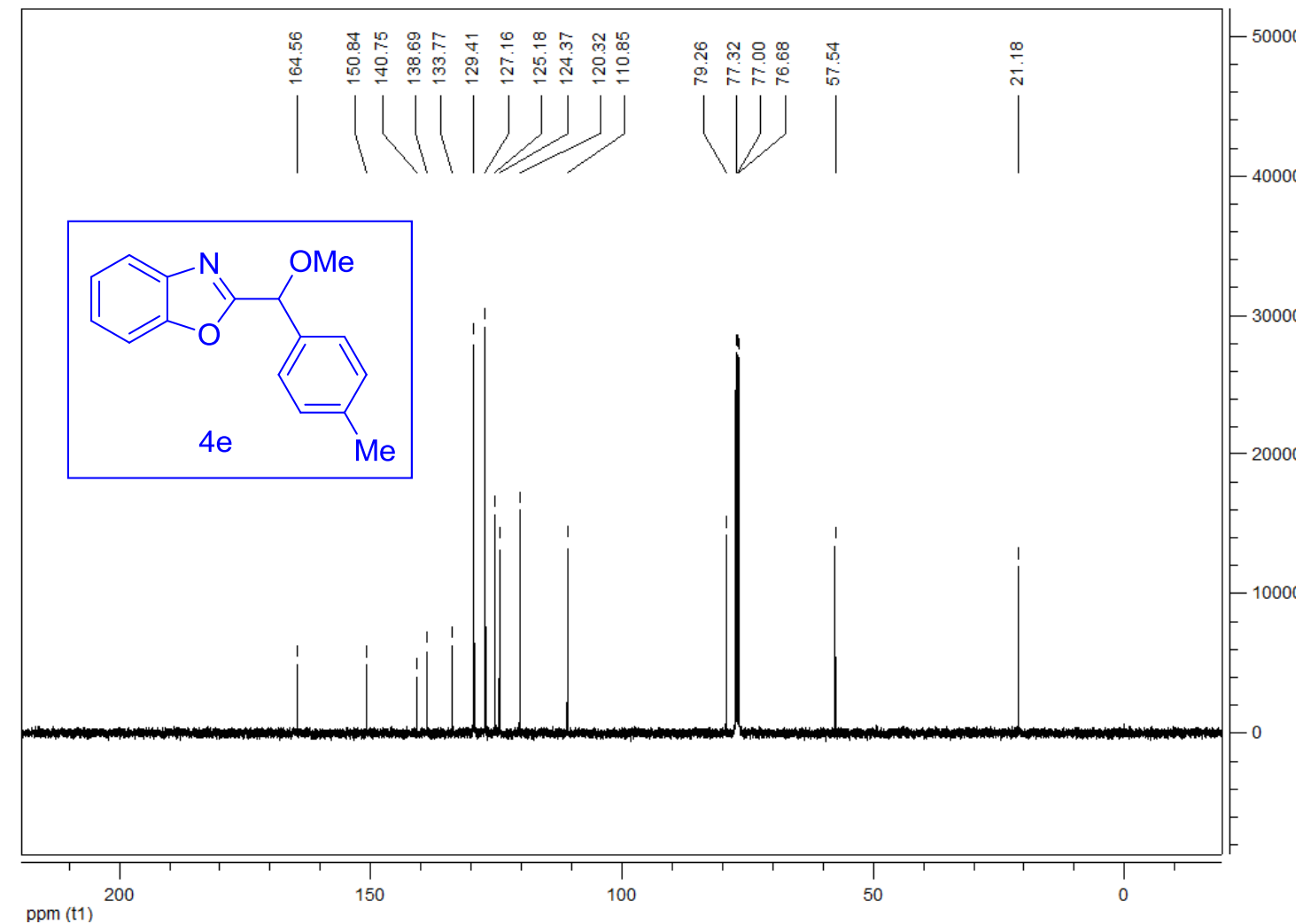


Figure S81. ${ }^{1}$ H NMR of 2-(methoxy(4-methoxyphenyl)methyl)benzo[d]oxazole (4f)

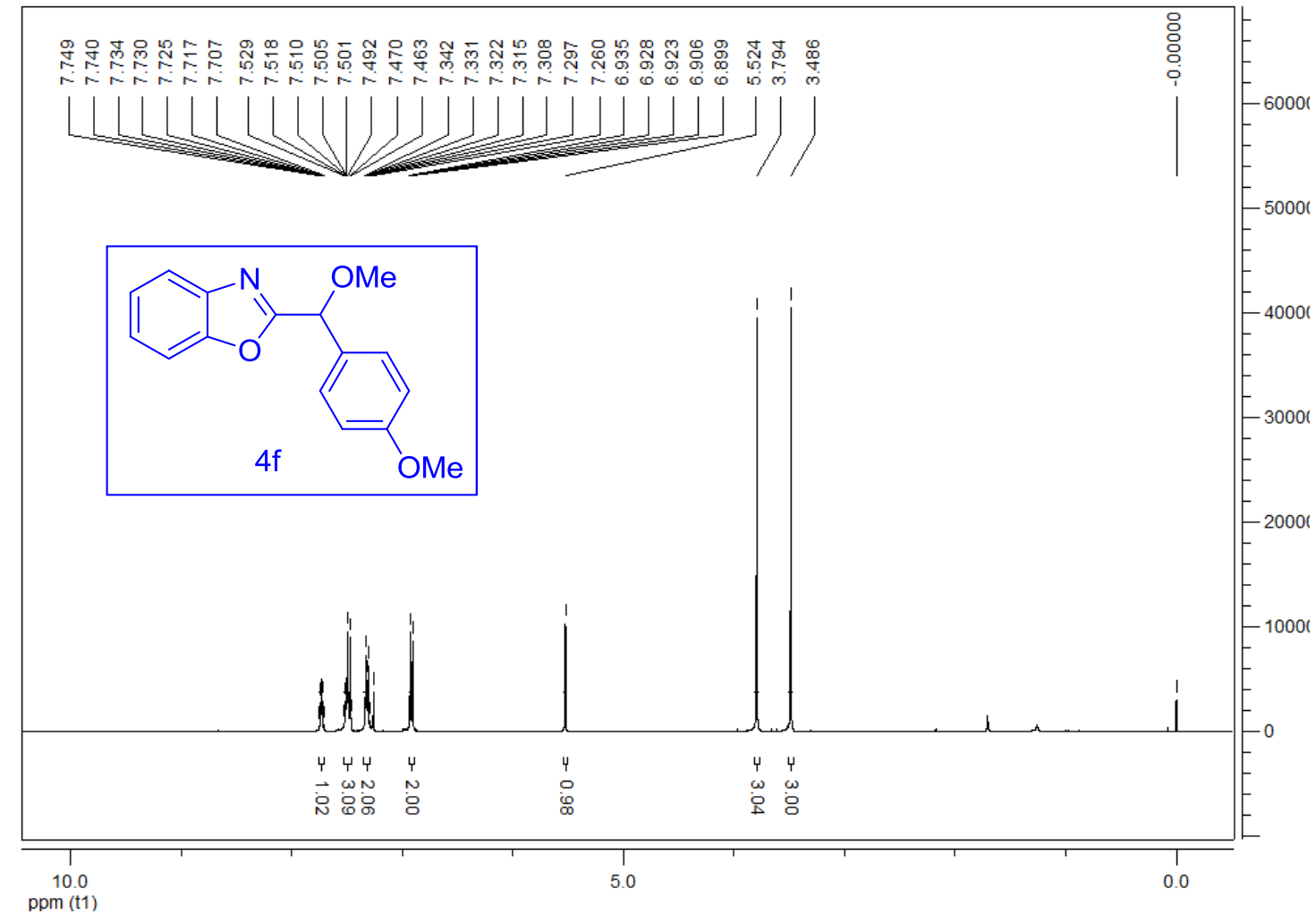

Figure S82. ${ }^{13} \mathrm{C}$ NMR of 2-(methoxy(4-methoxyphenyl)methyl)benzo[d]oxazole (4f)

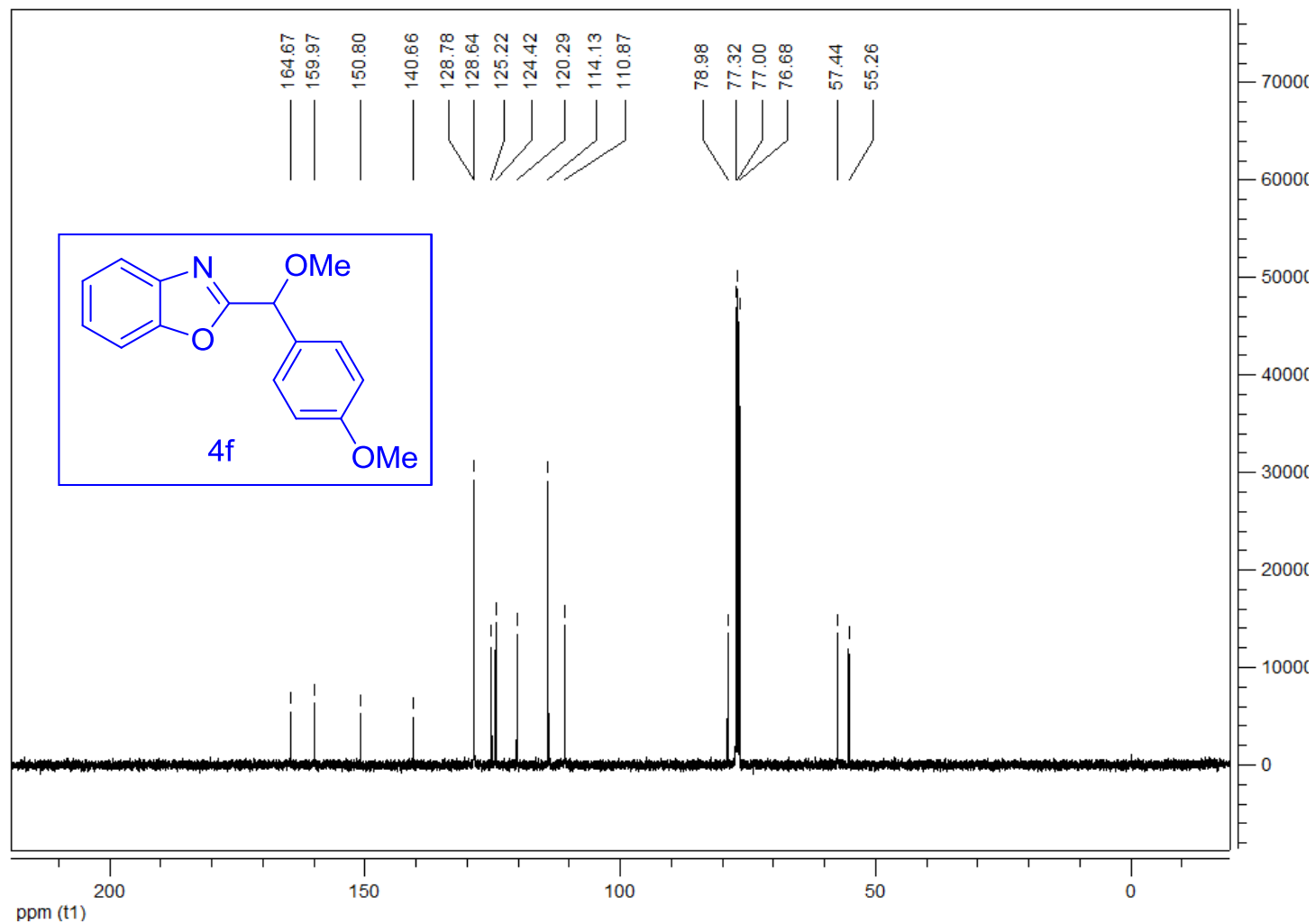


Figure S83. ${ }^{1} \mathrm{H}$ NMR of 2-([1,1'-biphenyl]-4-yl(methoxy)methyl)benzo[d]oxazole (4g)

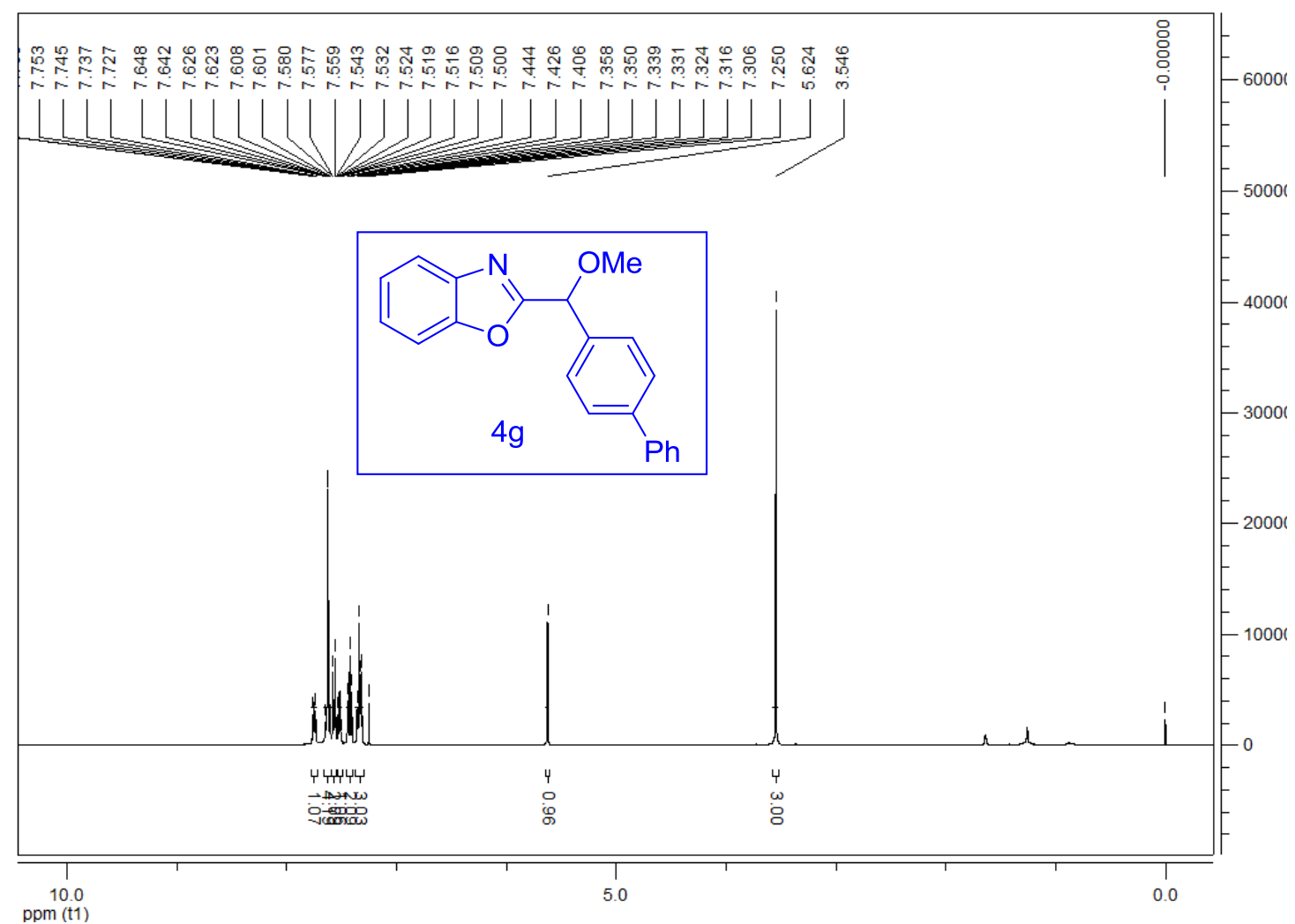

Figure S84. ${ }^{13} \mathrm{C}$ NMR of 2-([1,1'-biphenyl]-4-yl(methoxy)methyl)benzo[d]oxazole (4g)

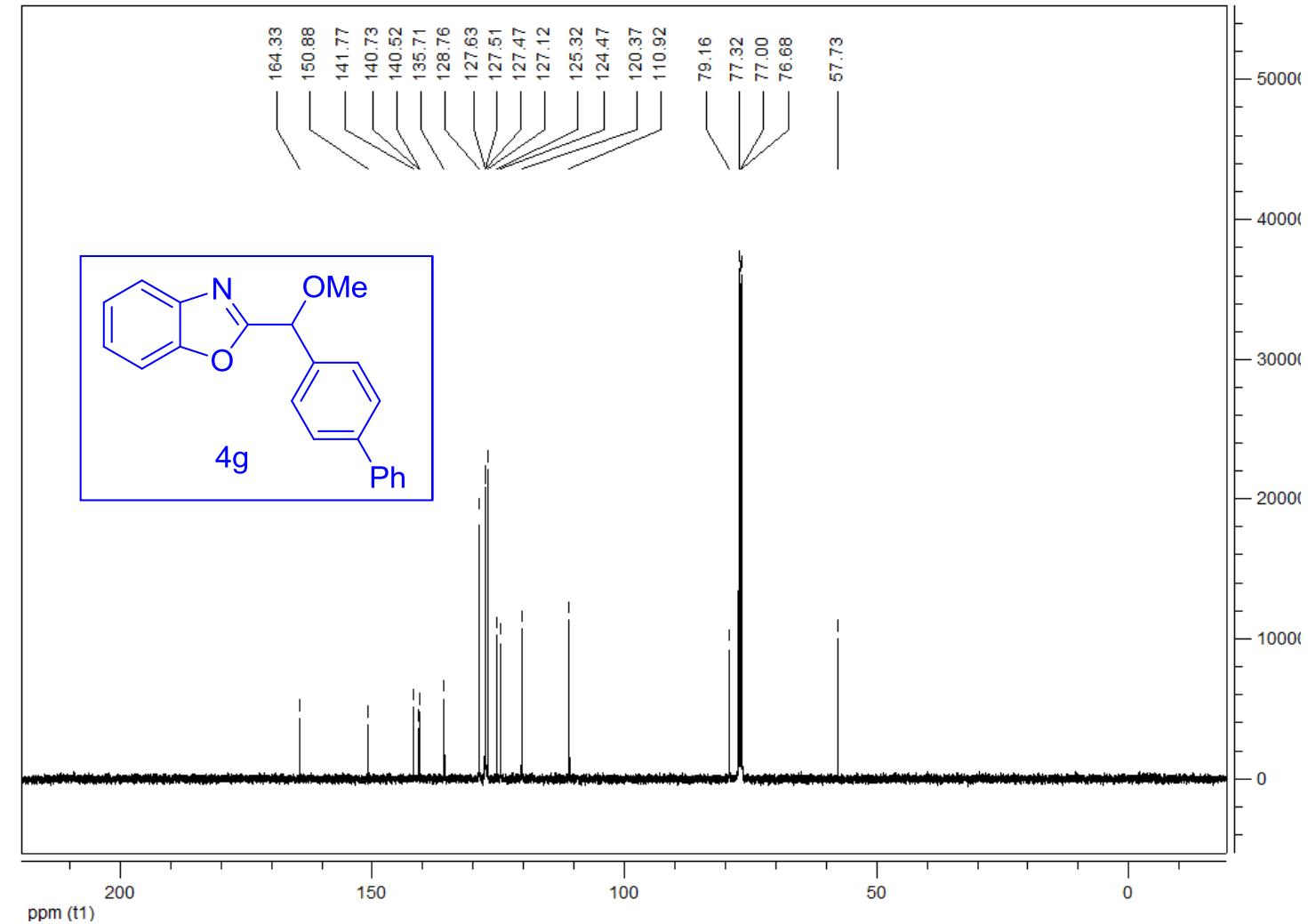


Figure S85. ${ }^{1} \mathrm{H}$ NMR of 2-((4-fluorophenyl)(methoxy)methyl)benzo[d]oxazole (4h)

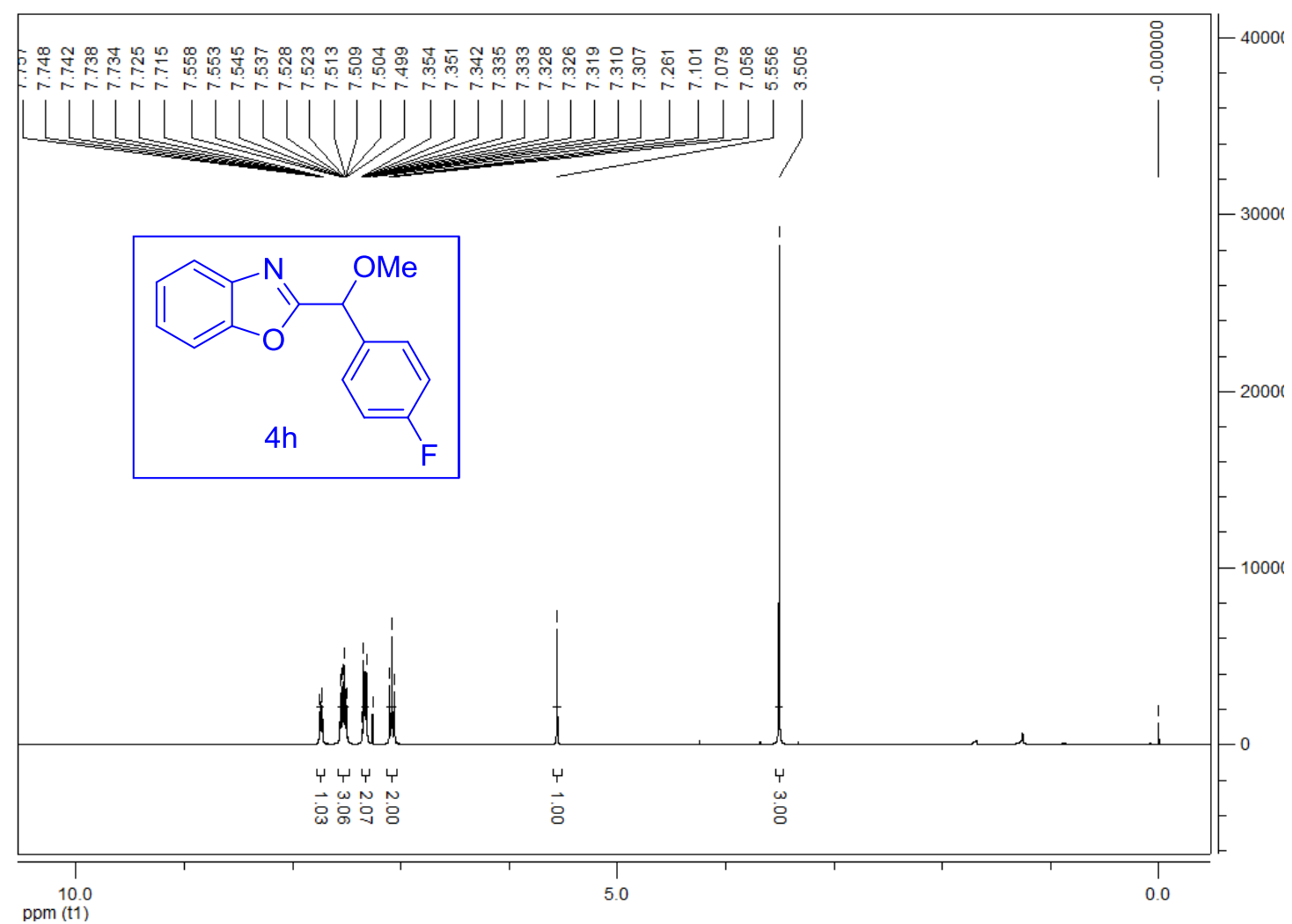

Figure S86. ${ }^{13} \mathrm{C}$ NMR of 2-((4-fluorophenyl)(methoxy)methyl)benzo[d]oxazole (4h)

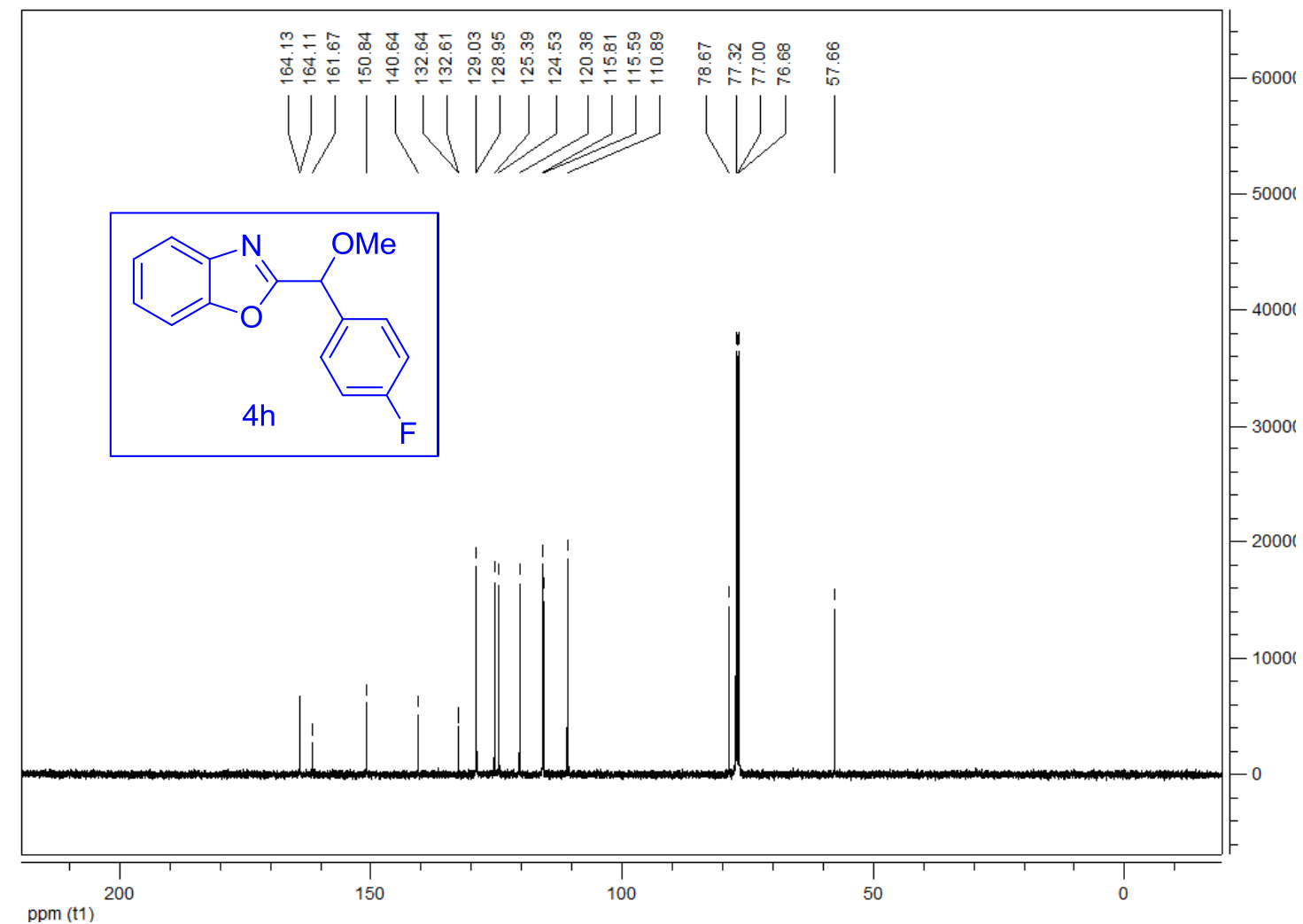


Figure S87. ${ }^{1}$ H NMR of 2-((4-chlorophenyl)(methoxy)methyl)benzo[d]oxazole (4i)

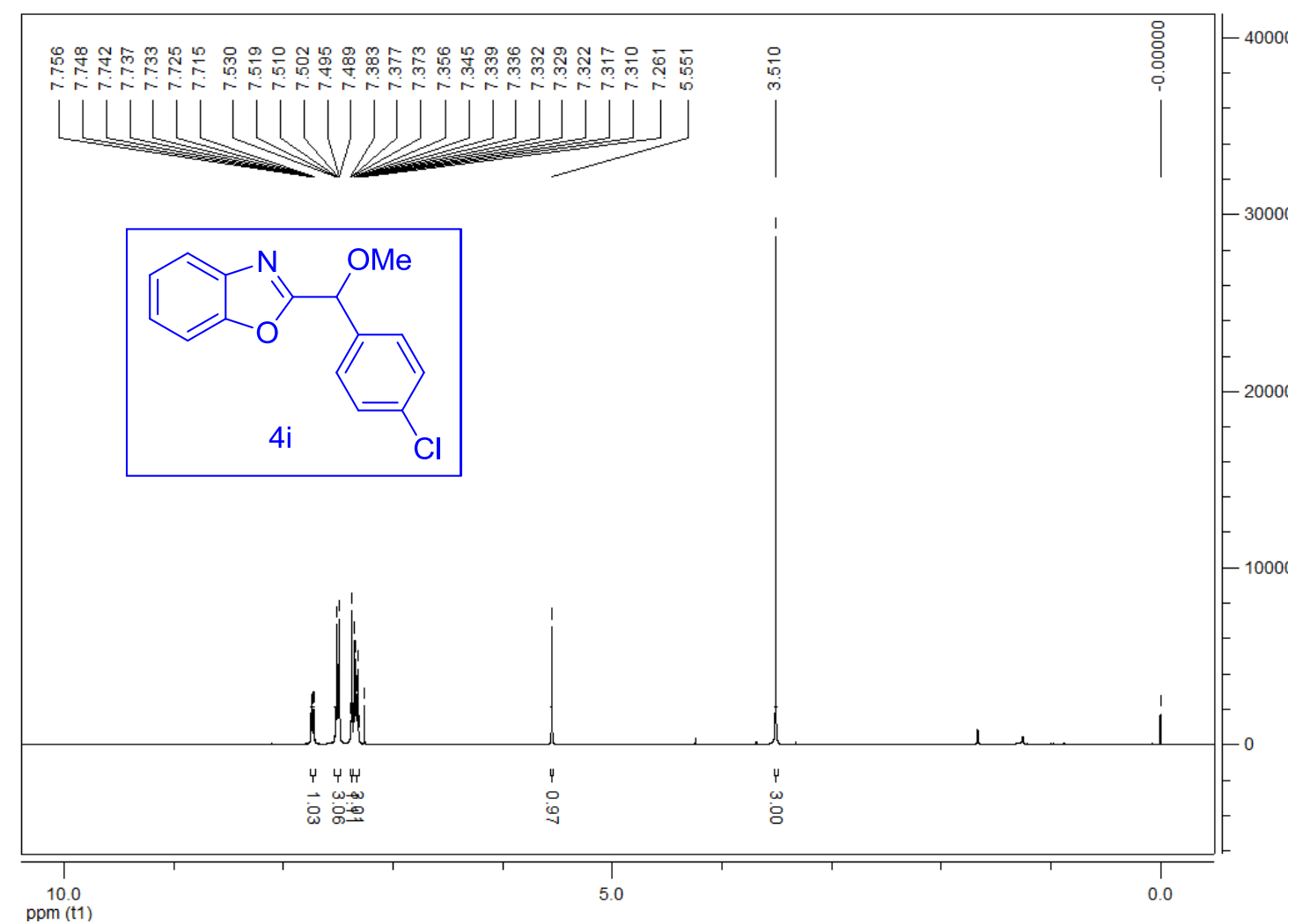

Figure S88. ${ }^{13} \mathrm{C}$ NMR of 2-((4-chlorophenyl)(methoxy)methyl)benzo[d]oxazole (4i)

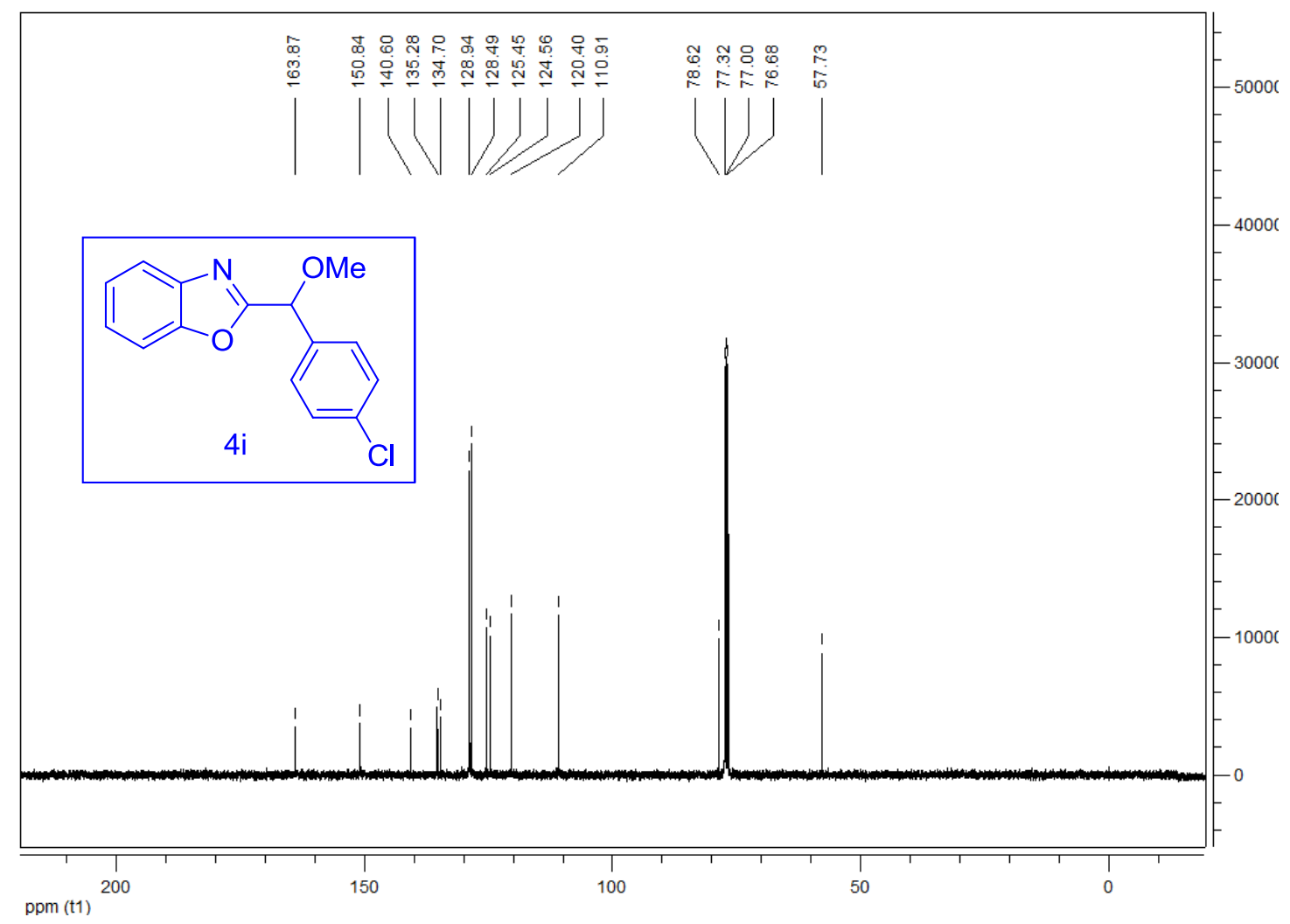


Figure S89. ${ }^{1}$ H NMR of 2-(methoxy(4-(trifluoromethyl)phenyl)methyl)benzo[d]oxazole $(4 \mathbf{j})$

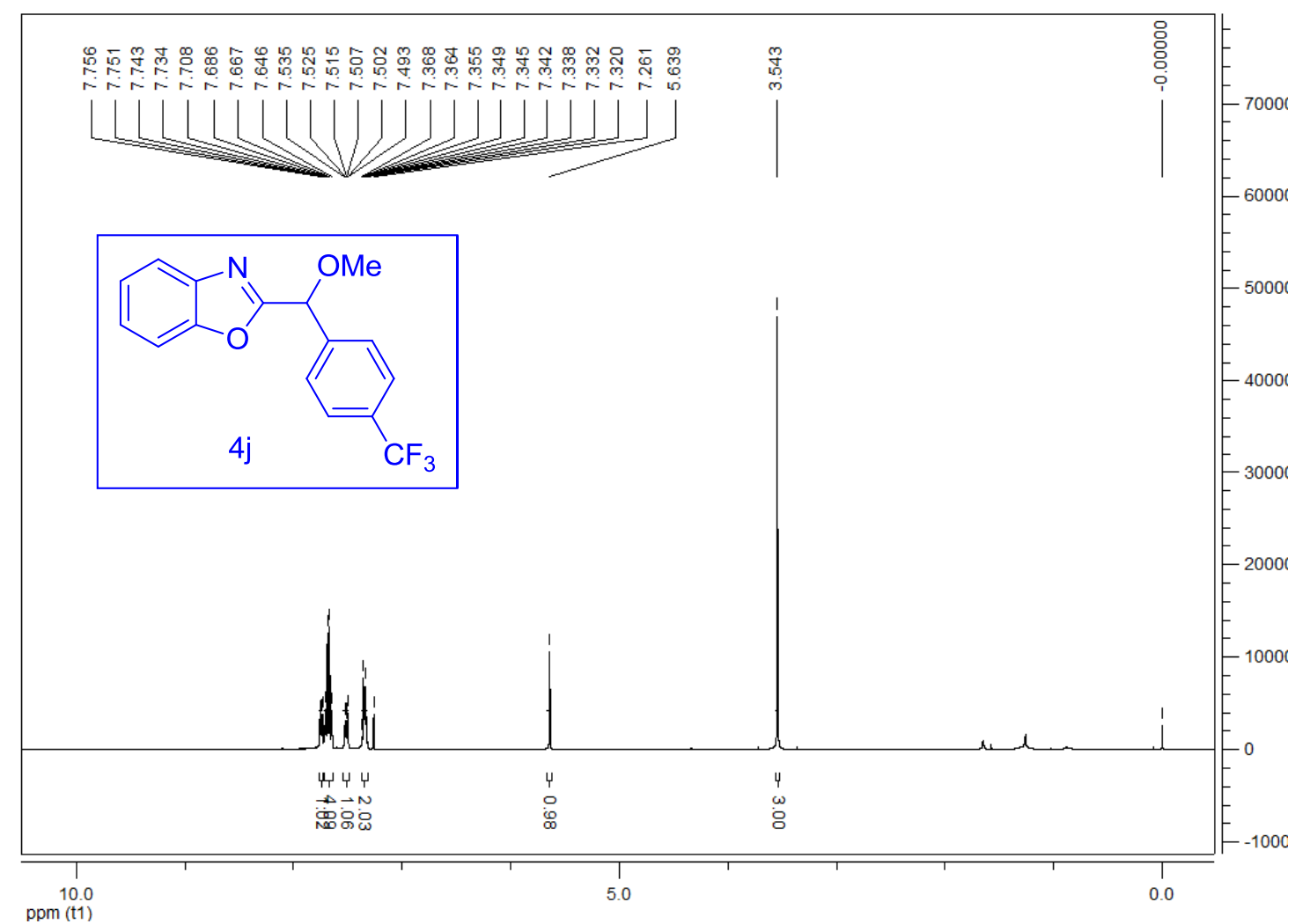

Figure S90. ${ }^{13} \mathrm{C}$ NMR of 2-(methoxy(4-(trifluoromethyl)phenyl)methyl)benzo[d]oxazole (4j)

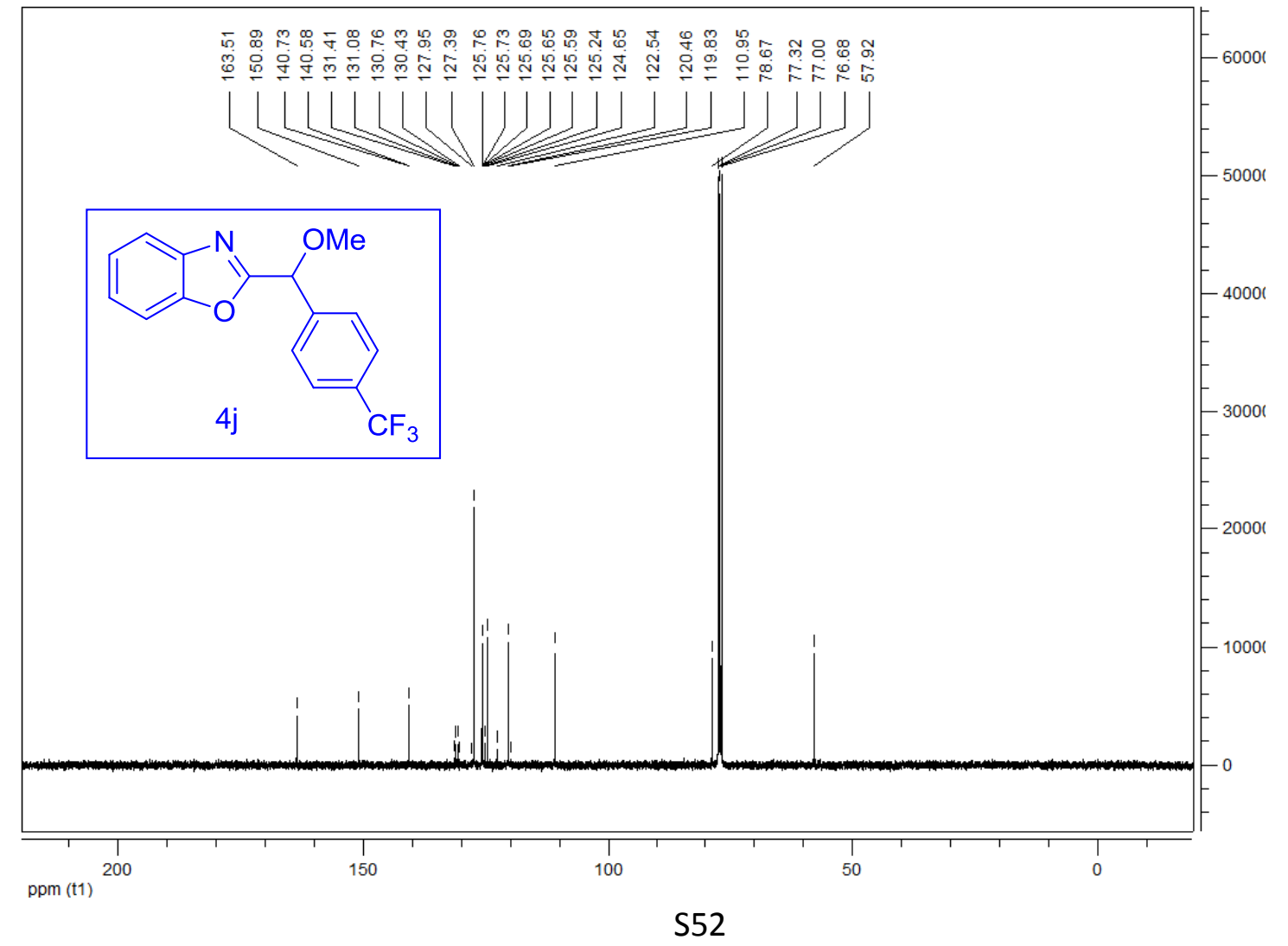


Figure S91. ${ }^{1}$ H NMR of 2-(furan-3-yl(methoxy)methyl)benzo[d]oxazole (4k)

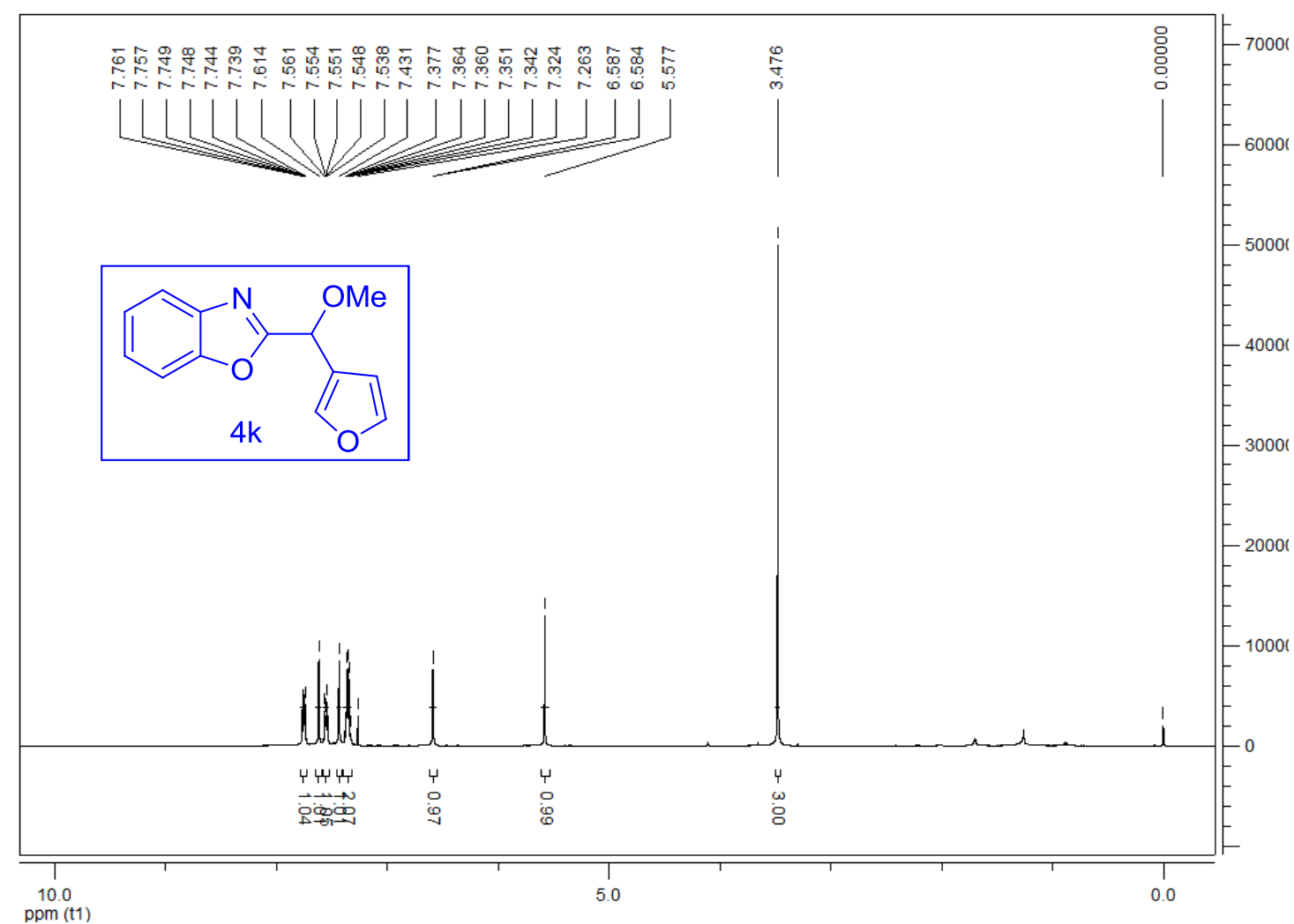

Figure S92. ${ }^{13} \mathrm{C}$ NMR of 2-(furan-3-yl(methoxy)methyl)benzo[d]oxazole (4k)

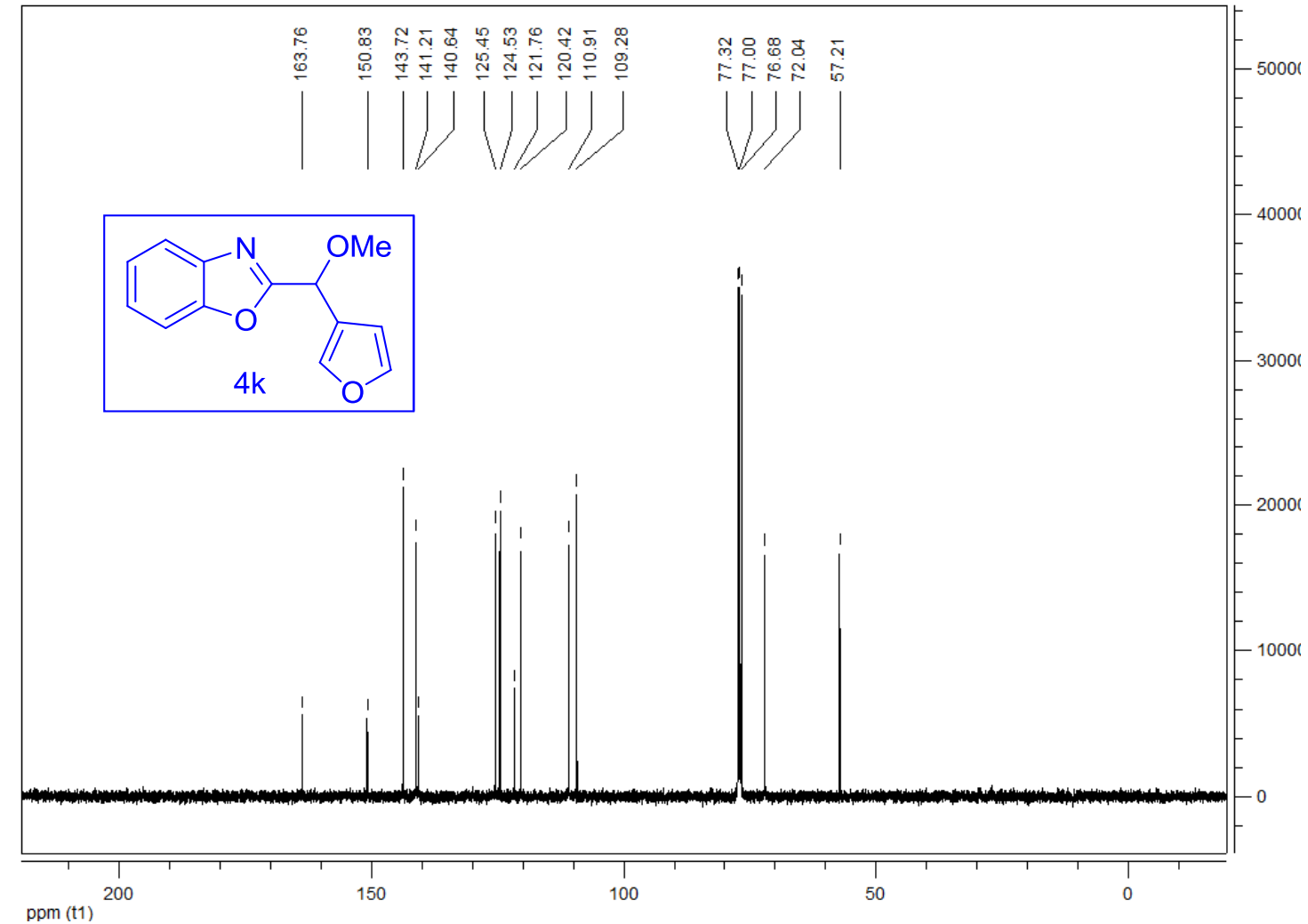


Figure S93. ${ }^{1}$ H NMR of 2-(methoxy(thiophen-2-yl)methyl)benzo[d]oxazole (4I)

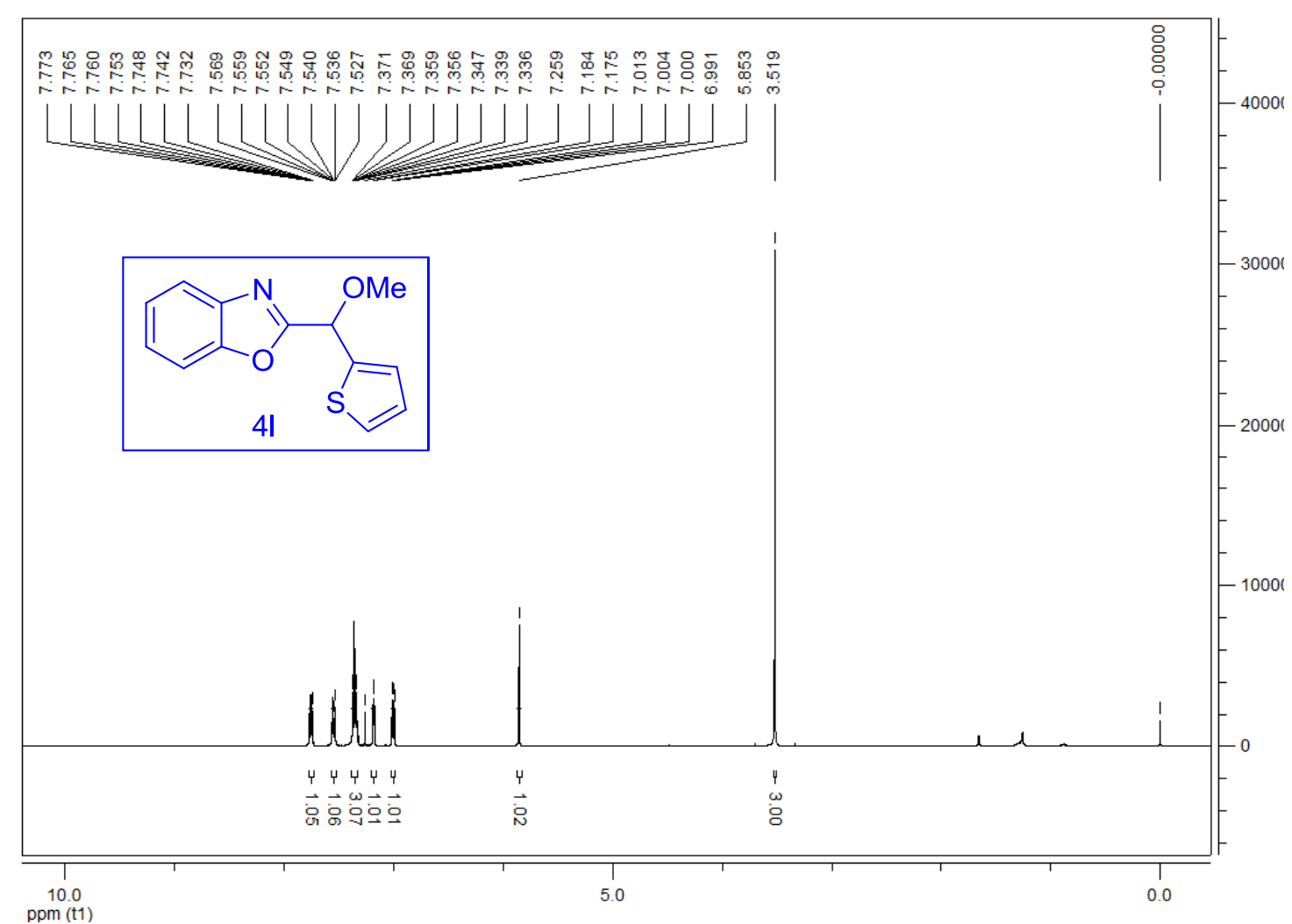

Figure S94. ${ }^{13} \mathrm{C}$ NMR of 2-(methoxy(thiophen-2-yl)methyl)benzo[d]oxazole (4l)

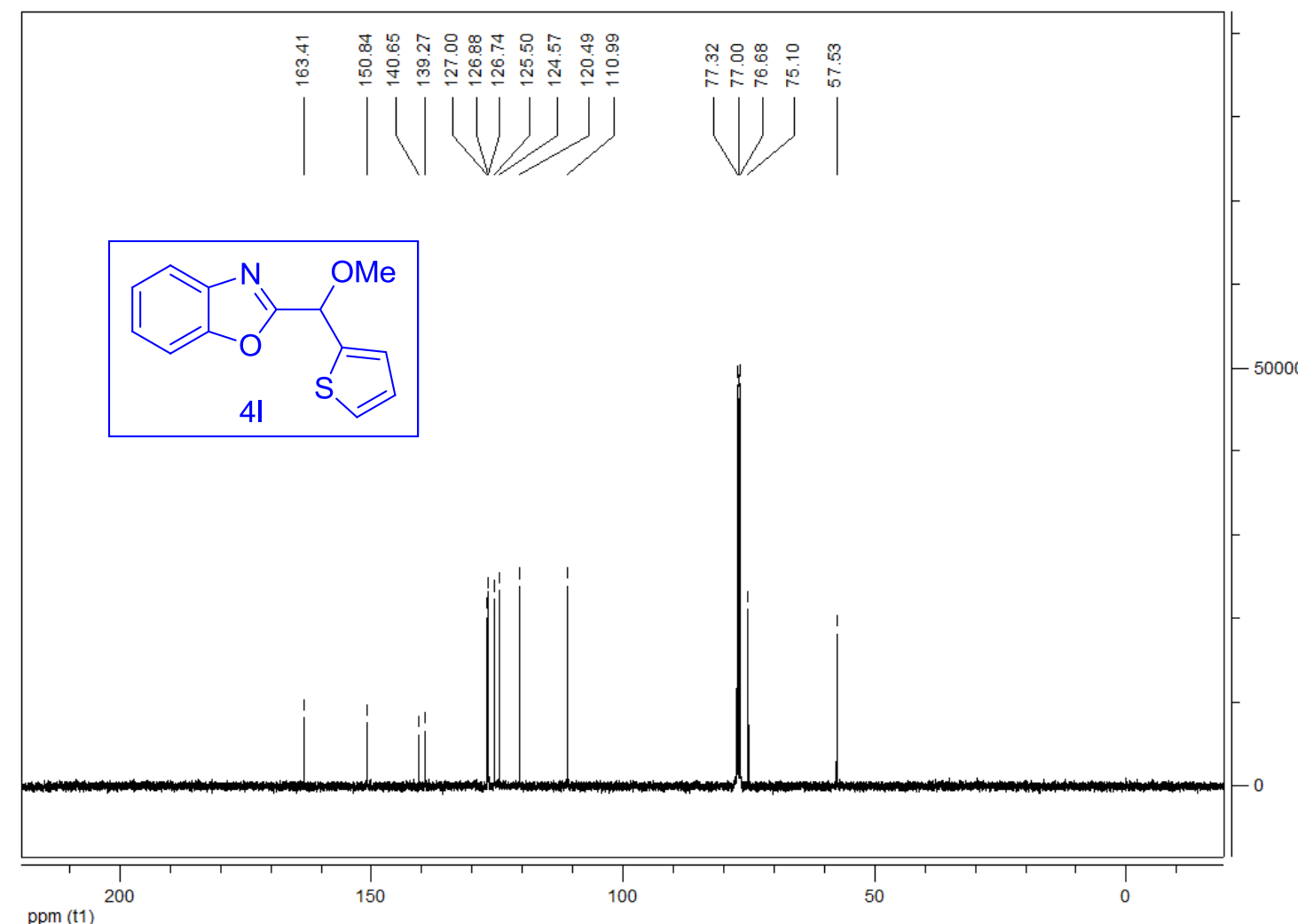


Figure S95. ${ }^{1} \mathrm{H}$ NMR of 2-(methoxy(naphthalen-2-yl)methyl)benzo[d]oxazole (4m)

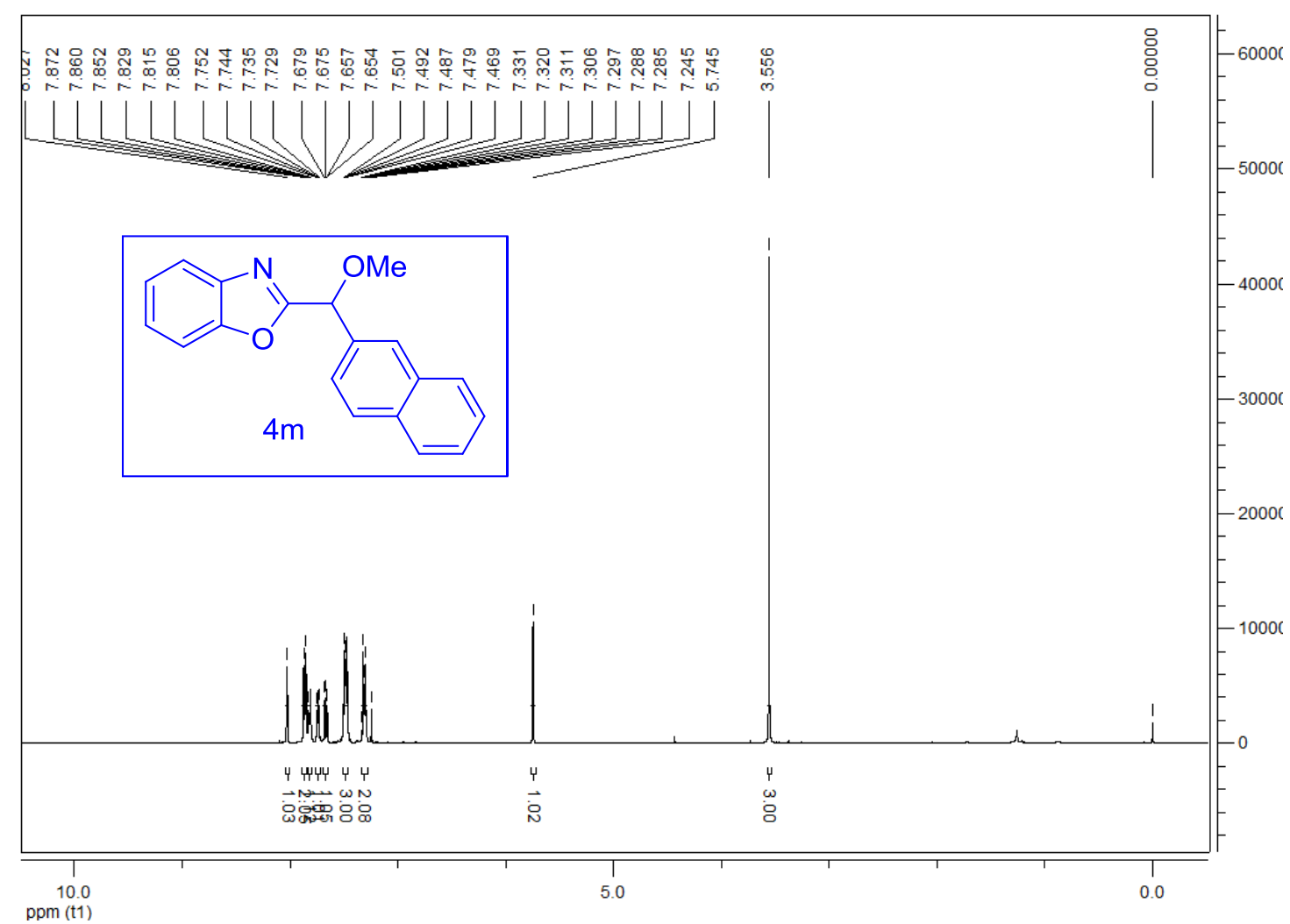

Figure S96. ${ }^{13} \mathrm{C}$ NMR of 2-(methoxy(naphthalen-2-yl)methyl)benzo[d]oxazole (4m)

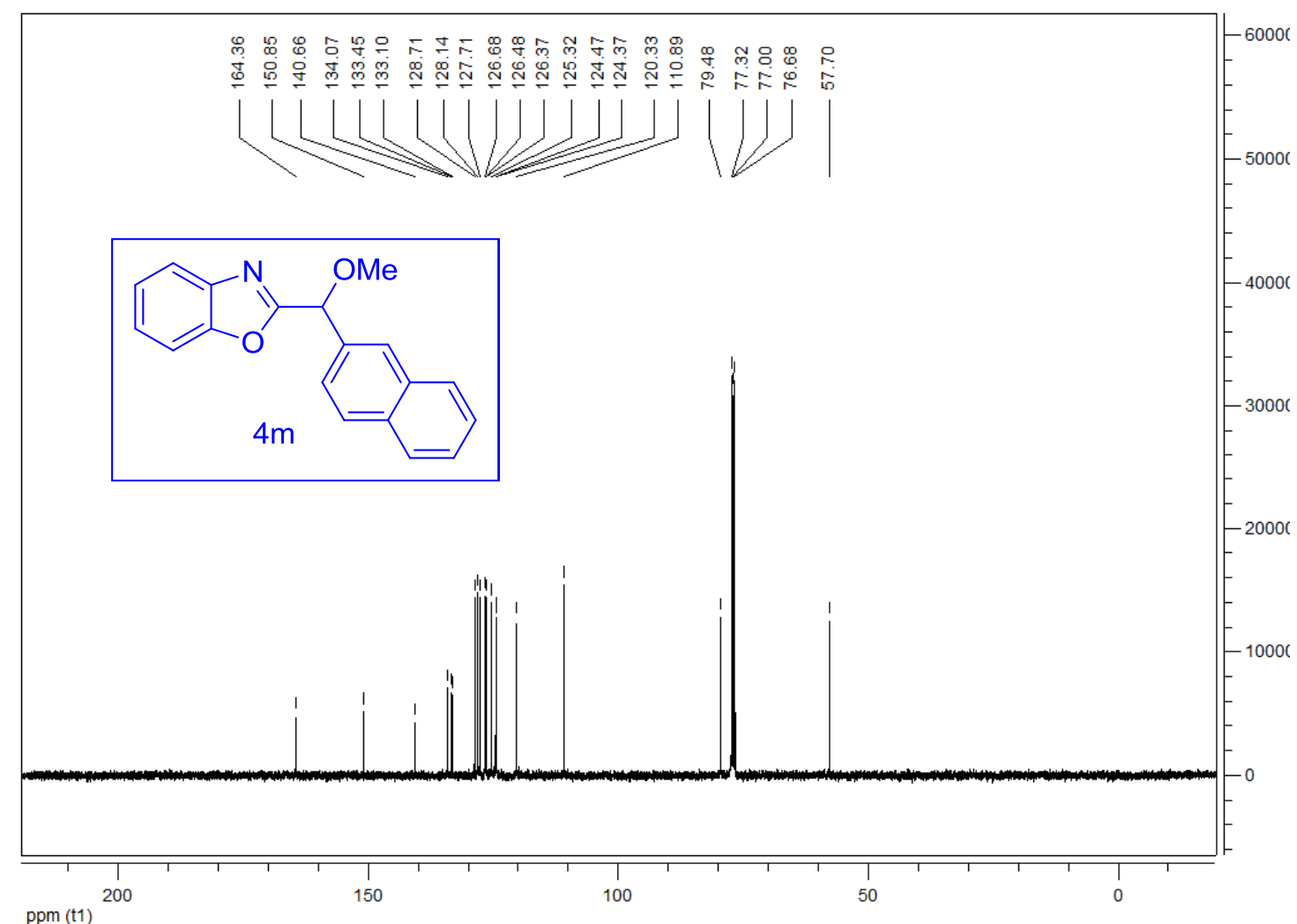


Figure S97. ${ }^{1}$ H NMR of 2-(ethoxy(phenyl)methyl)benzo[d]oxazole (4n)

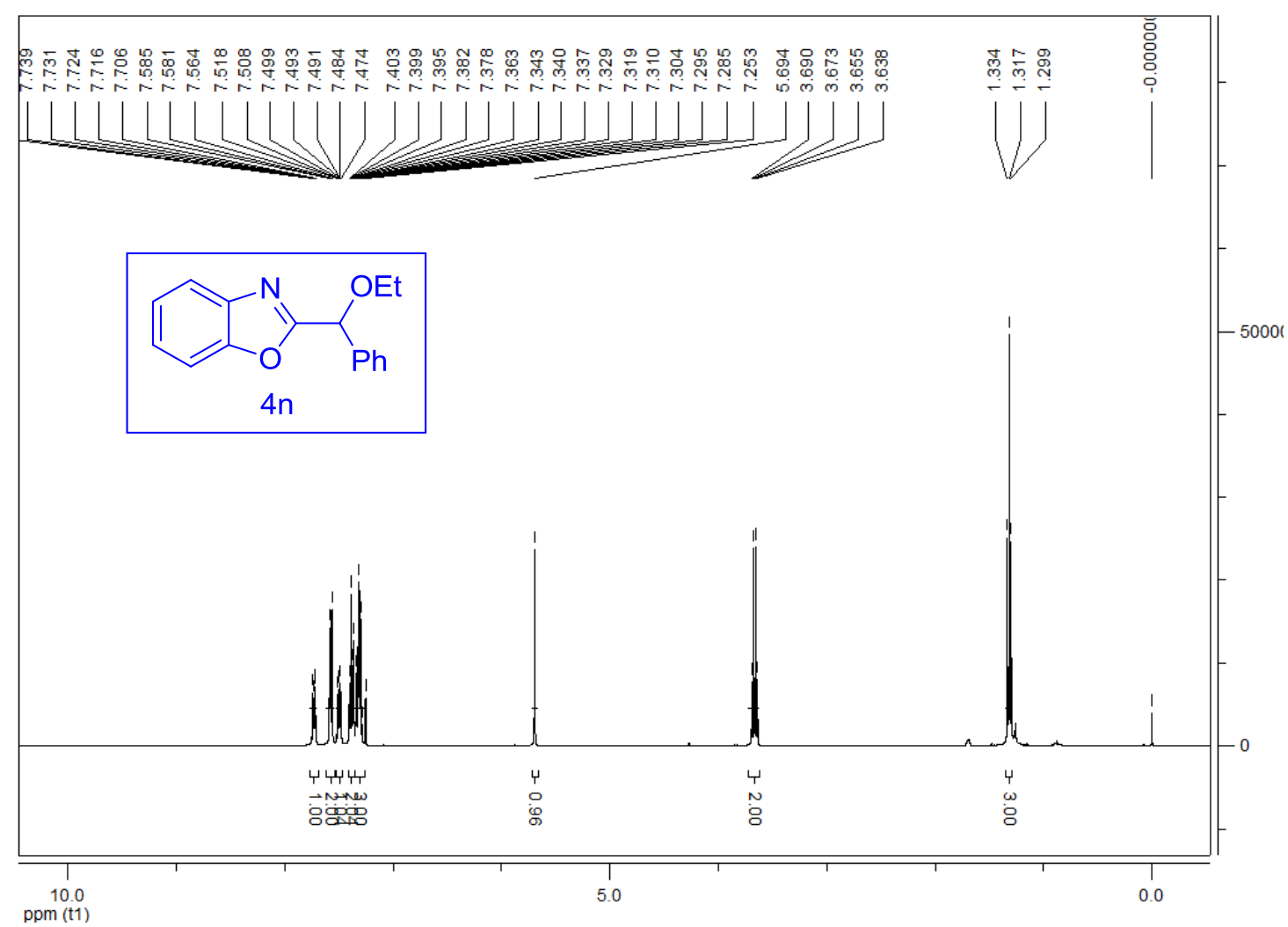

Figure S98. ${ }^{13} \mathrm{C}$ NMR of 2-(ethoxy(phenyl)methyl)benzo[d]oxazole (4n)

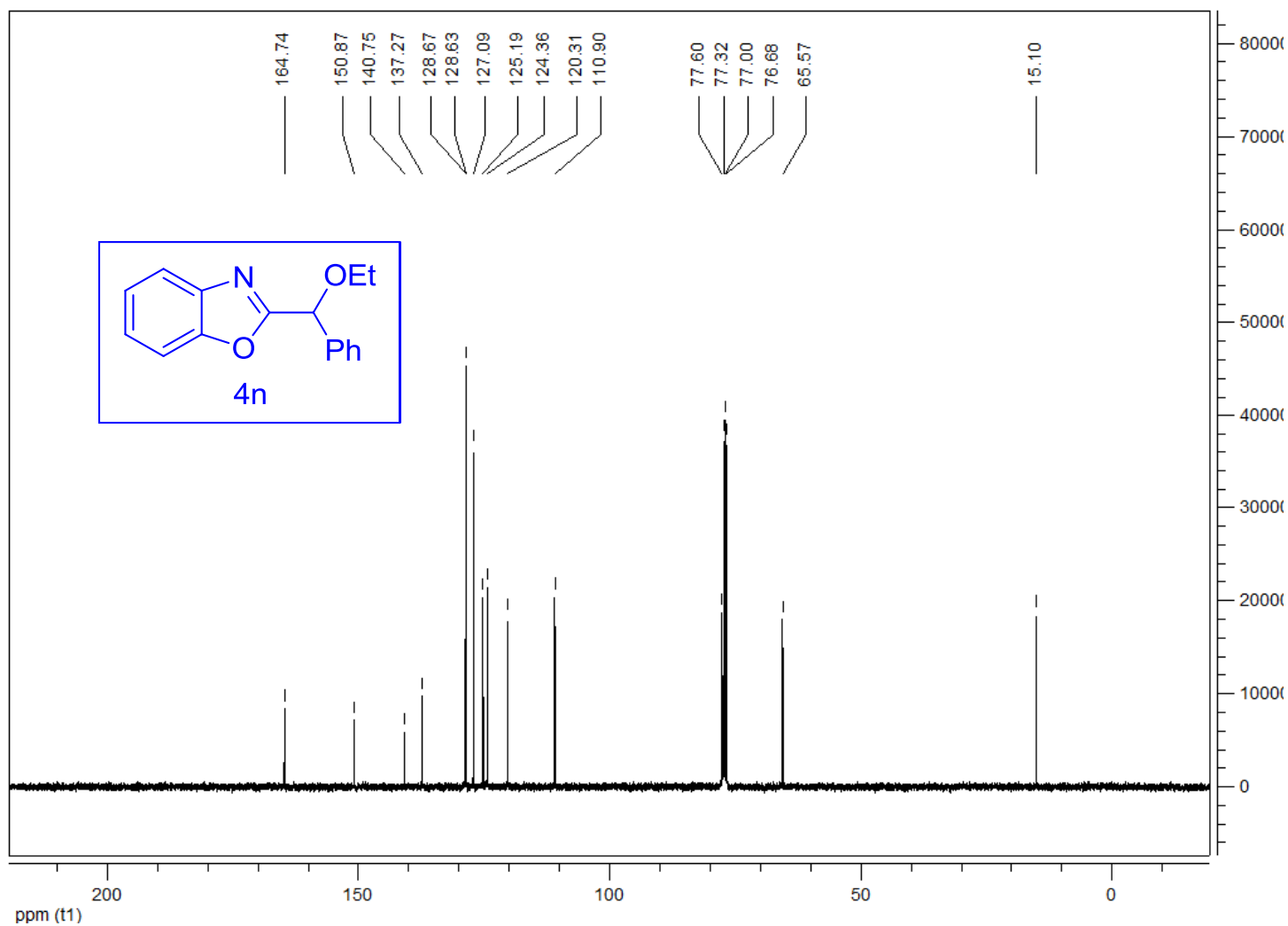

